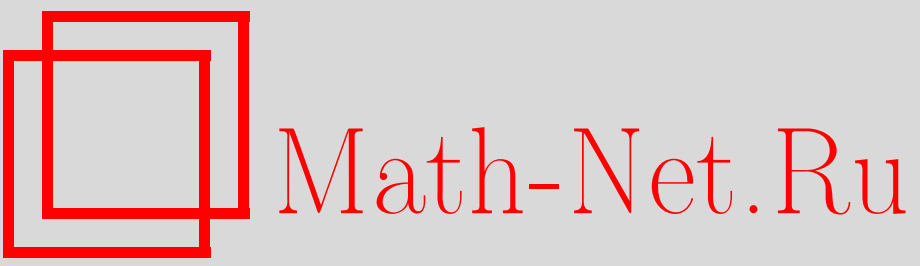

Н. И. Чернов, Д. Л. Лебовиц, Я. Г. Синай, Динамика массивного поршня, погруженного в идеальный газ, УМН, 2002, том 57, выпуск 6, 3-86

DOI: https://doi.org/10.4213/rm572

Использование Общероссийского математического портала Math-Net.Ru подразумевает, что вы прочитали и согласны с пользовательским соглашением

http://www.mathnet.ru/rus/agreement

Параметры загрузки:

IP: 54.197 .130 .99

26 апреля 2023 г., 13:44:21 


\title{
ДИНАМИКА МАССИВНОГО ПОРШНЯ, ПОГРУЖЕННОГО В ИДЕАЛЬНЫЙ ГАЗ
}

\author{
Л. ЛЕБОвиц, Я. СинАЙ, Н. ЧЕРнов
}

\begin{abstract}
Мы изучаем динамическую систему, состоящую из массивного поршня в кубическом сосуде болшшого размера $L$, заполненном идеальным газом. Поршень имеет массу $M \sim L^{2}$ и подвергается упругим столкновениям с $N \sim L^{3}$ невзаимодействующими частицами газа массы $m=1$. Мы показьваем, что при подходящих начальных условиях в пределе $L \rightarrow \infty$ существует некоторый скейлинг времени и пространства, в котором движение поршня и одночастичная функция распределения газа удовлетворяют автономной системе уравнений (гидродинамическим уравнениям) такой, что траектория механического движения поршня сходится по вероятности к решению системы гидродинамических уравнений на определенном отрезке времени. Мь также на эвристическом уровне обсуждаем динамику системы на больших временах.

Библиография: 26 названий.
\end{abstract}

\section{СОДЕРЖАНИЕ}

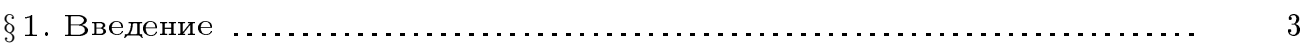

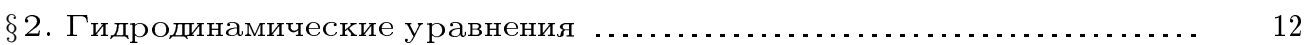

$\S 3$. Динамика перед первым перестолкновением $\quad \ldots \ldots \ldots \ldots \ldots \ldots \ldots \ldots \ldots . . \ldots 27$

$\S 4$. Динамика между первым и вторым перестолкновениями $\ldots . \ldots \ldots \ldots . .48$

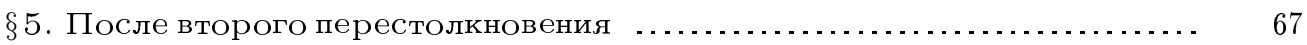

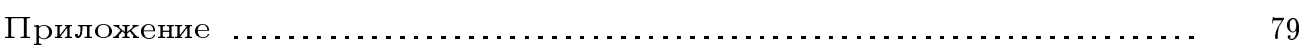

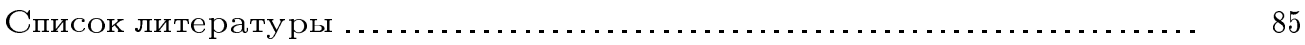

\section{$\S$ 1. Введение}

Эволющия макроскопической системы, состоящей из газа в сосуде, разделенном массивной подвижной стенкой (поршнем), является старой проблемой статистической физики, имеющей яркую историю. Она обсуждалась Ландау и Лифшицем [18], а позже - Лебовицем [20], Фейнманом [9], Кубо [17], см. недавние обзоры Либа [21], Грубера [10], а также [15].

В своей простейшей форме модель состоит из изолированного цилиндра, заполненного газом и разделенного на две части большим поршнем, который может свободно двигаться вдоль оси цилиндра, см. рис. 1. Вначале поршень зафиксирован при помоши зажима, а газ в каждой из частей эволющионирует независимо от газа в другой 
части и находится в равновесии ${ }^{1}$. Обозначим плотность и температуру газа в левой и правой частях $n_{L}, T_{L}$ и $n_{R}, T_{R}$ соответственно. Газ оказьвает давление (=сила на единицу площади) на поршень, которое задается в равновесной статистической механике как функция плотности и температуры: $P_{L}=P\left(n_{L}, T_{L}\right)$ и $P_{R}=P\left(n_{R}, T_{R}\right)$ слева и справа соответственно. В момент времени $t=0$ зажим отпускается и поршень освобождается. Теперь желательно описать эволюцию этой системы, особенно ее предельное (финальное) состояние при $t \rightarrow \infty$.

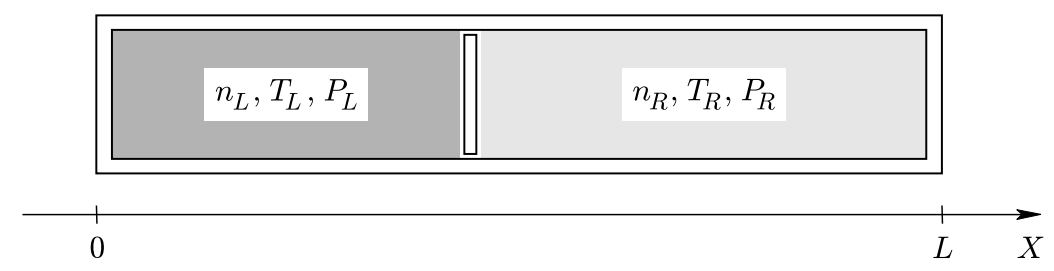

Рис. 1. Поршень в цилиндре, заполненном газом

Начиная с $P_{L} \neq P_{R}$ поршень движется под действием разности давлений и сжимает ту часть газа, давление в которой меньше, до тех пор, пока это давление не станет больше и будет толкать поршень назад. В зависимости от начальных значений $n_{L}$, $T_{L}, n_{R}, T_{R}$ и динамических характеристик газов поршень может совершать сложные движения, двигаясь взад и вперед, но постепенно остановится в том месте, где давление с обеих сторон будет одинаковьм. В это время ожидается также, что газ в каждой части будет находиться в равновесии.

Мы замечаем, однако, что равенство $P_{L}=P_{R}$ и тот факт, что газ в каждой части отдельно находится в равновесии, не гарантируют равенства $T_{L}=T_{R}$. В частности, для разреженных газов (которые мы будем рассматривать впредь) давление связано с плотностью и температурой соотношением $P=n k_{B} T$, где $k_{B}$-постоянная Больцмана, и, таким образом, возможно, что $T_{L}<T_{R}$, в то время как $n_{L}>n_{R}$, так что газ в левой части холоднее и плотнее, а в правой - горячее, но более разрежен (или наоборот). Точные значения температур $T_{L}$ и $T_{R}$ в тот момент времени, когда больше не сушествует разности давлений слева и справа и поршень останавливается, зависят от начальных условий и других характеристик системы, см., например, [7], [10]. Следовательно, мы имеем две возможности. Если $T_{L}=T_{R}$ и $P_{L}=P_{R}$, то тогда система как целое будет в равновесии и мы скажем, что она пришла к тепловому равновесию. Напротив, если $P_{L}=P_{R}$, но $T_{L} \neq T_{R}$, то говорят, что система находится в механическом равновесии или квазиравновесии.

\footnotetext{
${ }^{1}$ Для изолированной системы из $N$ атомов и общей энергией $E$ равновесное распределение определяется как равномерное распределение вероятностей $\rho$ еq на энергетической поверхности $E=$ const в фазовом пространстве ( $\rho$ еq назьвается микроканоническим ансамблем, по теореме Лиувилля оно инвариантно относительно динамики). Можно сказать, что эта система (газ) находится "в равновесии", если ее состояния "типичны" для меры $\rho$ еq. В этом состоянии макроскопический газ будет иметь (приближенно для болшших $N$ ) равномерную пространственную плотность и максвелловское распределение скоростей. Последнее означает, что компоненты $x$, $y, z$ векторов скорости являются нормальными случайными величинами $\mathcal{N}\left(0, \sigma^{2}\right)$ с одной и той же дисперсией $\sigma^{2}=k_{B} T / m$, где $k_{B}$ - постоянная Больцмана, $T$ - температура газа (которая является функцией $E$, см. ниже), $m$ - масса атома.
} 
Теперь можно поставить вопрос, будет ли механическое равновесие устойчивым в том смысле, что может длиться все время (в предположении, что вся система остается совершенно изолированной от внешнего мира), или газы найдут пути обмена энергией через поршень и постепенно приведут систему к тепловому равновесию. В некоторых учебниках утверждается на основе упрошенной интерпретации законов термодинамики, см. ниже, что механическое равновесие может оставаться “вечно", историю вопроса можно найти в [10], [11].

С другой стороны, Ландау и Лившиц [18], Фейнман [9] и многие другие на интуитивном уровне доказывали, что система должна от механического равновесия прийти к тепловому равновесию. Они предсказьвали, что более холодная часть будет постепенно нагреваться, а более горячая - охлаждаться, в то время как поршень будет медленно двигатся от более холодного края к более горячему при сохранении равенства давлений до тех пор, пока температура частей не выравняется и поршень не остановится.

Неясность, связанная с тем, как происходит эволющия газа после установления механического равновесия, сводится к следующему. Теплопроводность через стенку обычно ассоциируется с внутренним движением молекул стенки, сталкивающихся с молекулами газа и, тем самьм, обменивающихся импульсом и энергией. Однако в нашей идеализированной модели поршень и стенки предполагаются жесткими, твердыми и бесструктурными телами, и атомы газа сталкиваются с ними упругим образом. Эта идеализация в точности является причиной того, почему газы в различных частях могут быть в равновесии при различных температурах при закрепленном поршне. Незакрепленньй же поршень взаимодействует с атомами газа как целое, т.е. как одна большая массивная молекула. Поэтому поршень совершает мельчайшие микроскопические движения (вибрации), вызванные столкновениями с атомами с обеих сторон. Следовательно, некоторьй микроскопический обмен импульсом и энергией имеет место. Но эти микроскопические вибрации поршня не являются частью макроскопической термодинамики, в которой действие поршня на газ в каждой части рассматривается как внешняя механическая сила. При этом условии (и в предположении, что поршень сам по себе обладает энтропией) второй закон термодинамики предсказывает, что энтропия газа при переходе из некоторого начального равновесного состояния к конечному равновесному состоянию не может убывать. Когда газ в каждой части находится в равновесии, его термодинамическая энтропия, как известно [18], [3; Appendix C], будет равна

$$
S_{i}=N_{i}\left[-\log P_{i}+(1+3 / 2) \log T_{i}\right]+f\left(N_{i}\right), \quad i=L, R,
$$

где $N_{L}=n_{L} V_{L}, N_{R}=n_{R} V_{R}$ - число атомов в газах, а явный вид функции $f\left(N_{i}\right)$ для нас не важен, так как ее значение не меняется во времени. Если наша система эволюционирует от механического равновесия к тепловому, сохраняя при этом равенство давлений $P_{L}=P_{R}$ и общую кинетическую энергию $\frac{3}{2} k_{B}\left(N_{L} T_{L}+N_{R} T_{R}\right)$, тогда можно легко вычислить (мы оставляем это в качестве упражнения), что давление газов будет оставаться постоянным, а общая энтропия системы $S=S_{L}+S_{R}$ будет расти, пока не достигнет своего максимума в точке теплового равновесия. В то же самое время энтропия $S_{R}$ убьвает, пока $S_{L}$ увеличивается, так как $T_{L}$ падает и $T_{R}$ растет, а давление $P_{L}=P_{R}$ остается постоянным. Это убывание энтропии, как уже 
отмечалось, нарушало бы второй закон термодинамики, если бы поведение поршня описьвалось в рамках чистой механики, см. дальнейшие обсуждения в [21], [7] и критические замечания в [10], [11].

Следовательно, эволюция этой системы после достижения механического равновесия не может быть описана макроскопической термодинамикой (ввиду утверждения, что любая эволюция в изолированной системе не уменьшает общую энтропию). Действительная эволюция является результатом переноса микроскопической энергии между газами через столкновения с поршнем. Этот процесс является чисто микроскопическим и, в некотором смысле, противоречит интуищии, как мы сейчас объясним. Под действием столкновений с атомами газа с обеих сторон поршень вибрирует, т.е. покачивается взад и вперед. Когда поршень двигается в сторону более горячей части, атомы горячего газа отскакивают от поршня с возрастающей скоростью и, таким образом, получают энергию, в то время как атомы холодного газа сталкиваются с поршнем и замедляются, следовательно, теряют некоторую энергию. Когда поршень движется в сторону холодного края, все происходит наоборот. Так как, в среднем, горячий газ должен охлаждаться, а холодньй газ - нагреваться, то можно заключить, что движения поршня в направлении холодного края преобладают. С другой стороны, поршень должен медленно двигаться в направлении горячего края для того, чтобы обеспечить равенство давлений, см. вьше, так что должны преобладать смещения поршня в направлении горячего газа. Не вполне ясно, как эти кажущиеся противоположньми смешения ухитряются сосушествовать. Некоторые физики говорят в шутку о "конспиративном сговоре" между микроскопическими вибрациями поршня и приходящими атомами газов [11], [12]. По словам Каллена [3; Apendix C] “способная двигаться адиабатическая стенка представляется уникальной проблемой, содержащей много тонкостей".

Для того чтобы понять механизм переноса тепла через поршень, находяшийся в механическом равновесии $\left(P_{L}=P_{R}\right)$, обычно рассматривают простейший газ из невзаимодействующих частиц, т.е. идеальный газ. Еще в 1959 году Лебовищ [20] изучал поршень, взаимодействующий с двумя бесконечньми резервуарами, заполненными идеальными газами при различных температурах $T_{L} \neq T_{R}$. Его поршень также взаимодействовал с внешним потенциалом, например, пружиной, и, следовательно, мог прийти к некоторому нестационарному неравновесному состоянию под влиянием бесконечных резервуаров. Он использовал приближенное описание с помощью марковского процесса и обнаружил, что распределение скорости поршня является максвелловским и соответствует некоторой промежуточной температуре $T \in\left(T_{L}, T_{R}\right)$. Такое распределение приводило к систематическому переносу тепла между газами. Недавно Грубер и другие [12], [11], [25] использовали кинетическую теорию для изучения свободно движушегося поршня массы $M$, взаимодействующего с двумя бесконечными объемами идеальных газов из атомов массы $m \ll M$, находящихся при одинаковом давлении, но разных температурах. Они применили разложение уравнения Больцмана по $\varepsilon=m / M$, чтобы показать, что некоторьй макроскопический поток тепла проходит через поршень при $T_{L} \neq T_{R}$ и, значит, система постоянно приближается к тепловому равновесию. Ими также было найдено стационарное распределение скорости поршня, среднее значение которого задается соотношением

$$
\langle V\rangle=\frac{\sqrt{2 \pi m}\left(\sqrt{k_{B} T_{R}}-\sqrt{k_{B} T_{L}}\right)}{4 M}+o\left(\frac{m}{M}\right)
$$


(и не зависит от плотностей газа). Мы замечаем, что при $T_{L}<T_{R}$ среднее $\langle V\rangle>0$, что подтверждает наше предыдушее наблюдение о том, что поршень двигается от более холодного края к более теплому.

Уравнение (1.1) показьвает, что средняя скорость поршня отлична от нуля (хотя всего лишь порядка $O(m / M))$, несмотря на абсолютное равенство давлений $P_{L}=P_{R}$. Заметим, однако, что по сравнению с макроскопическим размером поршня отношение $m / M$ столь мало, что время, необходимое для того, чтобы поршень прошел какое-либо заметное расстояние, намного превосходит возраст Вселенной [11], и, следовательно, явление нельзя наблюдать экспериментально.

Мы делаем вывод о том, что эволюция системы происходит в два различных этапа. Первьй из них - это сходимость к механическому равновесию, которая происходит относительно быстро и скорость которой, в принципе, может быть вычислена из макроскопических уравнений. Второй этап - это переход от механического равновесия к тепловому. Этот процесс очень медленньй и значительно менее понят.

Кроме того, для идеального газа (атомы которого не взаимодействуют) возникает новая проблема. К моменту времени $t=0$, перед тем, как поршень освобождается, атомы газа движутся независимо друг от друга, каждый атом отскакивает от стенки и закрепленного поршня без обмена импульсом или энергией с другими атомами. Следовательно, распределение скорости не обязано быть максвелловским. Стационарное состояние идеального газа может быть описано любьм пуассоновским распределением с равномерной пространственной плотностью и симметрическим распределением скоростей. Например, половина атомов может двигаться в направлении поршня с единичной скоростью $v=1$, а другая половина - в противоположном направлении со скоростью $v=-1$, и такое состояние будет стационарньм. Однако сразу после освобождения поршня атомы начинают взаимодействовать друг с другом не прямо, а через столкновения с поршнем. Это открывает какой-то путь для обмена импульсом и энергией между атомами. Можно ожидать, что такие взаимодействия обязательно приведут к тепловому равновесию, когда распределение скоростей станет максвелловским, как мы объясняем в $\S 5$. Этот процесс, однако, может продолжаться даже дольше, чем установление равновесия средних кинетических энергий, описанное вьше.

Чтобы рассмотреть этот новьй процесс в “чистом” виде, мы предположим, что уже в начальньй момент система находится в однородном состоянии: плотность газа постоянна во всем цилиндре, а распределение скоростей одно и то же в обеих разделенных частях (но отлично от максвелловского). Тогда, казалось бы, нет никаких сил любой природы, которые сдвинули бы поршень в каком-либо направлении. В частности, когда поршень изначально помешен в середине цилиндра, тогда в силу симметрии не должно быть никакой причины для того, чтобы он как-либо двигался. С другой стороны, система не будет находиться в равновесии до тех пор, пока распределение скоростей не станет максвелловским, и, следовательно, должны сушествовать пути эволющии в направлении установления равновесия, приводящие к изменению макроскопического состояния системы. Мы обсудим это далее в $§ 5$.

Можно также рассматривать, как это было сделано в [20], [12], [25], более простой случай, когда сосуд имеет бесконечную длину по обе стороны от поршня и идеальные газы с обеих сторон содержат бесконечное число атомов. В этом случае проблема сводится к классическому рэлеевскому газу - большой массивной частище, погружен- 
ной в идеальный газ. В частности, наш поршень становится одномерньм рэлеевским газом, который мы опишем более подробно.

Пусть тяжелая свободная частица (назьваемая молекулой) массы $M$ движется вдоль прямой под действием упругих столкновений с атомами массы $m$ идеального газа, имеющего равномерную плотность $n$ и некоторое распределение скоростей $f(v) d v$. Обозначим через $X(t)$ и $V(t)=\dot{X}(t)$ положение и скорость молекулы в момент времени $t$. Хотя $f(v)$ не обязано быть максвелловским, скорость $V(t)$ и координата $X(t)$ могут быть приближены некоторыми гауссовскими стохастическими процессами:

ТеОРема 1.1 (Холли, [13]). Пусть плотность $f(v)$ является симметрической функиией, $f(v)=f(-v)$, и имеет конечный четвертый момент, $\int v^{4} f(v) d v<\infty$.

Тогда для любого конечного $t_{0}<\infty$ функиия $V(t) \sqrt{M}$ на отрезке $\left[0, t_{0}\right]$ сходится по распределению $\kappa$ некоторому процессу Орнштейна-Уленбека $\mathscr{V}_{t}$ при $M, n \rightarrow \infty u M / n \rightarrow$ const в то время, как $X(t) \sqrt{M}$ сходится $\kappa$ некоторому прочессу Орнштейна-Уленбека $\mathscr{X}_{t}$.

Процесс Орнштейна-Уленбека $\left(\mathscr{X}_{t}, \mathscr{V}_{t}\right)$ определяется [24] как

$$
d \mathscr{X}_{t}=\mathscr{Y}_{t} d t, \quad d \mathscr{V}_{t}=-a \mathscr{V}_{t} d t+\sqrt{D} d \mathscr{W}_{t},
$$

где $a>0, D>0$ - постоянные, $\mathscr{W}_{t}$ - винеровский процесс. Процесс Орштейна-Уленбека $\mathscr{X}_{t}$ сходится в подходящем пределе (например, при $a \rightarrow \infty, a^{2} / D=$ const) к винеровскому процессу.

Мы замечаем, что типичная скорость молекулы $V(t)$ имеет порядок $O(1 / \sqrt{M})$, что согласуется с равнораспределением энергии в системе, состояшем в том, что средние энергии всех частиц равны, т.е. $M\left\langle V^{2}\right\rangle=m\left\langle v^{2}\right\rangle=m \int v^{2} f(v) d v$.

Дюрр, Голдштейн и Лебовиц [8] обобшили предыдушую теорему на произвольную размерность и на асимметрическое распределение скоростей. Главная техническая трудность в доказательстве этой теоремы возникает из-за так назьваемых перестолкновений, которьй случаются, когда атом сталкивается с молекулой более одного раза. Перестолкновения приводят к внутренним автокоррелящиям в процессе $(X(t), V(t))$, которьй в противном случае был бы марковским. Доказательство, по сушеству, состоит в оценке нежелательного эффекта перестолкновений и в том, что этот эффект исчезает в пределе $M \rightarrow \infty$. Когда газ заперт в конечном цилиндре, эффект перестолкновений, однако, становится решаюшим. Все атомы будут двигаться к стенкам, отскакивать от них и двигаться назад к поршню, сталкиваясь с ним снова и снова. При этом возникают автокоррелящии. Не существует никакой стандартной техники, позволяющей оценить эффект перестолкновений в общем виде, но нами достигнут частичньй прогресс в этом направлении, см. $\S 4$.

Итак, проблема поршня поднимает серьезные математические вопросы и даже приводит к путанище в физических теориях. "Пресловутьй поршень", как он известен среди физиков, недавно снова привлек к себе большое внимание благодаря серии работ [11], [12], [25], [19]. В то же самое время цельй ряд новых численных экспериментов привели к лучшему теоретическому пониманию динамики системы, однако они же породили новые вопросы. Мы подчеркиваем, что очень немногие строгие результаты 
существуют даже для идеальных газов, не считая упомянутых выше стохастических приближений рэлеевского типа для бесконечного цилиндра.

Мы изучаем поршень в конечном цилидре, заполненном идеальными газами. Так как газы являются идеальными, мы не нуждаемся в предположении о том, что распределение скоростей атомов является максвелловским. Так как автокорреляции, индуцированные перестолкновениями, представляют главную трудность, мы специфицируем начальное состояние таким образом, что в течение некоторого интервала времени каждый атом газа сталкивается с поршнем не более двух раз. Главная цель нашей работы состоит в строгом описании динамики поршня в течение этого интервала времени. Мы показьваем, что в подходяшем пределе эволюция газа и поршня сходится к некоторому детерминированному процессу, которьй удовлетворяет замкнутой системе дифференциальных уравнений. Предположения, которые мы здесь делаем, упрощают техническое рассмотрение, но отнюдь не сводят проблему к тривиальной. На самом деле, многие интригуюшие вопросы остаются открытыми в нашем контексте, и мы обсуждаем их в последних двух параграфах этой работы.

Точная постановка задачи и главные результаты. Рассмотрим кубическую область $\Lambda_{L}$ размера $L$, разделенную на две части способной двигаться стенкой (поршнем). Каждая часть области $\Lambda_{L}$ содержит газ из невзаимодействуюших частищ массы $m=1$. Эти частищы сталкиваются упругим образом с внешними (фиксированными) стенками области $\Lambda_{L}$ и с движушимся поршнем. Поршень имеет массу $M=M_{L}$ и движется вдоль оси $x$ под действием соударений с частицами газа с обеих сторон. Размер $L$ куба является большим параметром нашей модели, и нас интересует поведение при $L \rightarrow \infty$. Мы будем предполагать, что $M_{L}$ пропорциональна плошади поршня, т.е. $M \sim L^{2}$, а число $N$ частиц газа пропорционально объему куба $\Lambda_{L}$, т.е. $N \sim L^{3}$, в то время как скорости частищ имеют порядок единицы.

Положение поршня в момент времени $t$ определяется единственной координатой $X=X_{L}(t), 0 \leqslant X \leqslant L$, а его скорость задается как $V=V_{L}(t)=\dot{X}_{L}(t)$. Так как компоненты скоростей частищ, перпендикулярные оси $x$, не играют никакой роли в динамике поршня, мы можем предположить, что каждая частища имеет только одну координату, $x$, и только одну компоненту скорости, $v$, направленную вдоль оси $x$.

Когда какая-либо частица со скоростью $v$ сталкивается с поршнем, имеющим скорость $V$, их скорости после столкновения, $v^{\prime}$ и $V^{\prime}$ соответственно, задаются как

$$
\begin{aligned}
V^{\prime} & =(1-\varepsilon) V+\varepsilon v, \\
v^{\prime} & =-(1-\varepsilon) v+(2-\varepsilon) V,
\end{aligned}
$$

где $\varepsilon=2 m /(M+m)$. Мы предполагаем, что $M+m=2 m L^{2} / a$, где $a>0$-некоторая постоянная, так что

$$
\varepsilon=\frac{2 m}{M+m}=\frac{a}{L^{2}}
$$

Когда частица сталкивается со стенкой $x=0$ или $x=L$, ее скорость только меняет знак.

Эволющия системы тогда является полностью детерминистской, но нужно задать начальные условия. Мы будем предполагать, что поршень начинает двигаться из середины $\left(X_{L}(0)=L / 2\right)$ с нулевой скоростью $V_{L}(0)=0$ (см. также $\left.\S 2\right)$. Начальная 
конфигурация частищ газа выбирается случайно как реализация (двумерного) пуассоновского процесса на $(x, v)$-плоскости (с ограничением $1 \leqslant x \leqslant L$ ) с плотностью $L^{2} p_{L}(x, v)$, где $p_{L}(x, v)$ - функция, удовлетворяющая некоторым условиям (см. ниже), множитель $L^{2}$ равен площади сечения сосуда, ортогонального к оси $x$. Это означает, что для любой области $D \subset[0, L] \times \mathbb{R}^{1}$ число $N_{D}$ частищ газа $(x, v) \in D$ при $t=0$ имеет пуассоновское распределение с параметром

$$
\lambda_{D}=L^{2} \iint_{D} p_{L}(x, v) d x d v
$$

Для любых неперекрьваюшихся областей, скажем, $D_{1} \cap D_{2}=\varnothing$, соответствующие числа $N_{D_{1}}$ и $N_{D_{2}}$ статистически независимы. Мы отметим, что общее число частиц газа $N$ также является пуассоновской случайной величиной. Полная энергия и полньй начальньй импульс также случайны.

Пусть $\Omega_{L}$ обозначает пространство всех возможных конфигураций частицгаза в $\Lambda_{L}$ (т.е. все счетные подмножества в $[0, L] \times \mathbb{R}^{1}$ ). Для каждой реализации ${ }^{2} \omega \in \Omega_{L}$ траектория детерминистского поршня будет обозначаться $X_{L}(t, \omega)$, а его скорость $V_{L}(t, \omega)$.

Данная модель - это механическая модель, чьи динамические характеристики $X_{L}(t, \omega)$ и $V_{L}(t, \omega)$ зависят от большого параметра $L$ и для каждого $L$ случайны (зависят от $\omega$ ).

Для того чтобы получить детерминистское описание динамики поршня, нужно взять предел при $L \rightarrow \infty$ и одновременно перемасштабировать пространство и время. Мы вводим новые пространственные и временные координаты

$$
y=x / L \text { и } \tau=t / L,
$$

которые отвечают эйлерову скейлингу при гидродинамическом предельном переходе. Мы назьваем $y$ и $\tau$ макроскопическими ("медленными") переменными в противоположность исходным микроскопическим (“быстрым") переменным $x$ и $t$. Пусть

$$
Y_{L}(\tau, \omega)=X_{L}(\tau L, \omega) / L, \quad W_{L}(\tau, \omega)=V_{L}(\tau L, \omega)
$$

обозначают положение и скорость поршня в макроскопических переменных. Начальные условия тогда имеют вид: $Y_{L}(0)=X_{L}(0) / L=0.5, W_{L}(0)=V_{L}(0)=0$.

Теперь вполне естественно предположить, что начальная плотность $p_{L}(x, v)$ согласуется с нашим масштабированием:

$$
p_{L}(x, v)=\pi_{0}(x / L, v)
$$

где функция $\pi_{0}(y, v)$ не зависит от $L$. Без потери общности мы можем предположить, что $\pi_{0}$ нормирована так, что

$$
\int_{0}^{1} \int_{-\infty}^{\infty} \pi_{0}(y, v) d v d y=1 .
$$

\footnotetext{
${ }^{2}$ Технически возможно, чтобы две или более частиц сталкивались с поршнем одновременно, и тогда динамика уже не будет определена, но, как известно, кратные столкновения случаются с вероятностью нуль [13], так что мы будем их игнорировать.
} 
Тогда среднее число частиц во всем сосуде $\Lambda_{L}$ точно равно $L^{3}$ :

$$
\mathrm{E}(N)=\iint L^{2} p_{L}(x, v) d v d x=L^{3}
$$

где $\mathrm{E}(\cdot)$ - математическое ожидание.

Кроме того, мы предполагаем, что функция $\pi_{0}(y, v)$ удовлетворяет нескольким техническим требованиям, сформулированным ниже. Смысл и цель этих предположений станут ясны позже.

(P1) Гладкость. $\pi_{0}(y, v)$ - кусочно $C^{1}$-функция с равномерно ограниченньми частньми производными, т.е. $\left|\partial \pi_{0} / \partial y\right| \leqslant D_{1}$ и $\left|\partial \pi_{0} / \partial v\right| \leqslant D_{1}$ для некоторого $D_{1}>0$.

(P2) Линии разрыва. $\pi_{0}(y, v)$ может быть разрывной на линии $y=Y_{L}(0)$ (т.е. "на поршне"). Кроме того, она может иметь конечное число $\left(\leqslant K_{1}\right)$ других линий разрьва на $(y, v)$-плоскости со строго положительным наклоном (каждая линия задается уравнением $v=f(y)$, где $f(y)$ есть $C^{1}$-функция и $0<c_{1}<$ $\left.f^{\prime}(y)<c_{2}<\infty\right)$.

(Р3) Гранииы для плотности. Пусть

$$
\pi_{0}(y, v)>\pi_{\min }>0 \text { для } v_{1}<|v|<v_{2}
$$

при некоторых $0<v_{1}<v_{2}<\infty$ и

$$
\sup _{y, v} \pi_{0}(y, v)=\pi_{\max }<\infty .
$$

Требования (1.8) и (1.9) главньм образом означают, что $\pi_{0}(y, v)$ принимает значение порядка единищы.

(Р4) “Обрезание" скорости. Пусть

$$
\pi_{0}(y, v)=0, \text { если }|v|<v_{\min } \text { или }|v|>v_{\max }
$$

для некоторых $0<v_{\min }<v_{\max }<\infty$. Это значит, что скорость частиц газа ограничена сверху величиной $v_{\max }$, а снизу - величиной $v_{\min }$.

(P5) Приближенное равенство давлений. $\pi_{0}(y, v)$ должно быть почти симметричньм относительно поршня:

$$
\left|\pi_{0}(y, v)-\pi_{0}(1-y,-v)\right|<\varepsilon_{0}
$$

для всех $0<y<1$ и некоторого достаточно малого $\varepsilon_{0}>0$.

Требования (P4) и (Р5) являются решаюшими. Мы увидим, что благодаря им скорость поршня $\left|V_{L}(t, \omega)\right|$ будет меньше минимальной скорости частиц газа с вероятностью, близкой к единице, при $t=O(L)$. Такие предположения были впервые сделаны в [19].

Мы считаем, что $D_{1}, K_{1}, c_{1}, c_{2}, v_{1}, v_{2}, v_{\min }, v_{\max }, \pi_{\min }$ и $\pi_{\max }$ в $(\mathrm{P} 1)-(\mathrm{P} 4)$ являются фиксированными (глобальными) постоянньми, а $\varepsilon_{0}$ в (Р5) - дополнительньй малый параметр. Мы будем предполагать в этой статье, что $\varepsilon_{0}$ достаточно мало, так что:

$$
\varepsilon_{0}<\bar{\varepsilon}_{0}\left(D_{1}, K_{1}, c_{1}, c_{2}, v_{1}, v_{2}, v_{\min }, v_{\max }, \pi_{\min }, \pi_{\max }\right) .
$$

Важно отметить, что для гидродинамического предела не требуется, чтобы $\varepsilon_{0} \rightarrow 0$. Параметр $\varepsilon_{0}$ остается положительным и фиксированным при $L \rightarrow \infty$.

Теперь мы формулируем наш главньй результат. 
Теорема 1.2. Существуют не зависящая от $L$ функиия $Y(\tau)$, определенная для всех $\tau \geqslant 0$, и положительное $\tau_{*} \approx 2 / v_{\max }$ (в действительности, $\tau_{*} \rightarrow 2 / v_{\max }$ при $\left.\varepsilon_{0} \rightarrow 0\right)$ maкue, что

$$
\sup _{0 \leqslant \tau \leqslant \tau_{*}}\left|Y_{L}(\tau, \omega)-Y(\tau)\right| \rightarrow 0
$$

$u$

$$
\sup _{0 \leqslant \tau \leqslant \tau_{*}}\left|W_{L}(\tau, \omega)-W(\tau)\right| \rightarrow 0
$$

по вероятности при $L \rightarrow \infty$. Здесь $W(\tau)=\dot{Y}(\tau)$.

Эта теорема устанавливает сходимость по вероятности случайных функций $Y_{L}(\tau, \omega), W_{L}(\tau, \omega)$, характеризуюших механическую эволюшию поршня, к детерминистским функциям $Y(\tau), W(\tau)$ в гидродинамическом пределе $L \rightarrow \infty$.

Функции $Y(\tau)$ и $W(\tau)$ удовлетворяют определенным дифференциальным уравнениям (типа Эйлера), устанавливаемьм в следующем параграфе. Эти уравнения имеют решения для всех $\tau \geqslant 0$, но мы можем гарантировать сходимость (1.12) и (1.13) только для $\tau<\tau_{*}$. Что происходит при $\tau>\tau_{*}$ и, в особенности, при $\tau \rightarrow \infty$, остается открытой проблемой. Некоторые численные результаты и эвристические наблюдения в этом направлении представлены в $\S 5$.

ЗАмЕчАнИя. Функщия $Y(\tau)$ принадлежит, по крайней мере, $C^{1}$ и, более того, кусочно $C^{2}$. На интервале $\left(0, \tau_{*}\right)$ ее первая производная $W=\dot{Y}$ (скорость) и ее вторая производная $A=\ddot{Y}$ (ускорение) останутся $\varepsilon_{0}$-малыми: $\sup _{\tau}|W(\tau)| \leqslant$ const $\cdot \varepsilon_{0}$ и $\sup _{\tau}|A(\tau)| \leqslant$ const $\cdot \varepsilon_{0}$, см. следуюший параграф̆.

Мы также оценим скорость сходимости в (1.12) и (1.13). Точнее, мы покажем, что сушествует $\tau_{0}>0\left(\tau_{0} \approx 1 / v_{\max }\right)$ такое, что

$$
\left|Y_{L}(\tau, \omega)-Y(\tau)\right|=O\left(\frac{\ln L}{L}\right)
$$

при $0<\tau<\tau_{0}$ и

$$
\left|Y_{L}(\tau, \omega)-Y(\tau)\right|=O\left(\frac{\ln L}{L^{1 / 7}}\right)
$$

при $\tau_{0}<\tau<\tau_{*}$. Те же самые оценки справедливы для $\left|W_{L}(\tau, \omega)-W(\tau)\right|$, см. $\S \S 3$ и 4 . Эти оценки справедливы с “подавляющей” вероятностью, в частности, они справедливы для всех $\omega \in \Omega_{L}^{*} \subset \Omega_{L}$ таких, что $\mathrm{P}\left(\Omega_{L}^{*}\right)=1-O\left(L^{-\ln L}\right)$.

\section{§ 2. Гидродинамические уравнения}

Уравнения, описьваюшие детерминистскую функцию $Y(\tau)$, содержат другую детерминистскую функцию - скейлинговую плотность газа $\pi(y, v, \tau)$. В начальньй момент $\pi(y, v, 0)=\pi_{0}(y, v)$, а для $\tau>0$ плотность $\pi(y, v, \tau)$ эволюционирует согласно следуюшим правилам.

(Н1) Свободное движение. Внутри сосуда плотность удовлетворяет стандартному уравнению непрерьвности для системы невзаимодействуюших частиц без внешних сил:

$$
\left(\frac{\partial}{\partial \tau}+v \frac{\partial}{\partial y}\right) \pi(y, v, \tau)=0
$$

для всех $y$, кроме $y=0, y=1$ и $y=Y(\tau)$. 
Уравнение (2.1) имеет простое решение

$$
\pi(y, v, \tau)=\pi(y-v s, v, \tau-s)
$$

для $0<s<\tau$ таких, что $y-v \tau \notin\{0, Y(\tau-r), 1\}$ для всех $r \in(0, s)$. Уравнение (2.2) по сравнению с уравнением (2.1) обладает одним преимуществом: оно применимо ко всем точкам $(y, v)$, включая те из них, где функция $\pi$ недифоференцируема.

(Н2) Столкновения со стенками. На стенках $y=0$ и $y=1$

$$
\begin{aligned}
& \pi(0, v, \tau)=\pi(0,-v, \tau) \\
& \pi(1, v, \tau)=\pi(1,-v, \tau)
\end{aligned}
$$

(Н3) Столкновения с поринем. На поршне $y=Y(\tau)$

$$
\begin{array}{lll}
\pi(Y(\tau)-0, v, \tau)=\pi(Y(\tau)-0,2 W(\tau)-v, \tau) & \text { для } \quad v<W(\tau) \\
\pi(Y(\tau)+0, v, \tau)=\pi(Y(\tau)+0,2 W(\tau)-v, \tau) & \text { для } \quad v>W(\tau)
\end{array}
$$

где $v$ - скорость после столкновения, а $2 W(\tau)-v$-перед столкновением; здесь

$$
W(\tau)=\frac{d}{d \tau} Y(\tau)
$$

есть (детерминистская) скорость поршня.

Остается описать эволюцию $W(\tau)$. Предположим, что в момент времени $t$ положение поршня есть $Y$, а его скорость $W$. Поршень находится под воздействием частиц $(y, v)$, ударяюших его справа (тогда $y=Y+0$ и $v<W$ ) и слева (тогда $y=Y-0$ и $v>W)$.

(H4) Скорость поршня. Скорость $W=W(\tau)$ поршня должна удовлетворять уравнениям

$$
\int_{W}^{\infty}(v-W)^{2} \pi(Y-0, v, \tau) d v=\int_{-\infty}^{W}(v-W)^{2} \pi(Y+0, v, \tau) d v
$$

см. также ниже дополнительное требование $\left(\mathrm{H} 4^{\prime}\right)$.

В физических терминах (2.7) означает равенство давлений: поршень "выбирает" скорости $W$ так, что давление входяших частиц уравновешивается извне. Уравнение (2.7) является инструментом для нашего детерминистского приближения динамики поршня.

Мы можем скомбинировать из двух интегралов в (2.7) один, определяющий плотность частиц, сталкиваюшихся с поршнем (“плотность на поршне”), следуюшим образом:

$$
q(v, \tau ; Y, W)= \begin{cases}\pi(Y+0, v, \tau) & \text { при } v<W \\ \pi(Y-0, v, \tau) & \text { при } v>W .\end{cases}
$$

Тогда (2.7) может быть переписано как

$$
\int_{-\infty}^{\infty}(v-W(\tau))^{2} \operatorname{sign}(v-W(\tau)) q(v, \tau ; Y(\tau), W(\tau)) d v=0 .
$$


Мы также замечаем, что для $\tau>0$, когда (2.5) справедливо,

$$
W(\tau)=\frac{\int v \pi(Y-0, v, \tau) d v}{\int \pi(Y-0, v, \tau) d v}=\frac{\int v \pi(Y+0, v, \tau) d v}{\int \pi(Y+0, v, \tau) d v}
$$

т.е. скорость поршня является средней скоростью соседних с ним частищ с каждой из сторон.

Система (гидродинамических) уравнений, заданных в (Н1)-(Н4), замкнута и для подходящих начальных условий, как мы вскоре увидим, должна полностью определять функции $Y(\tau), W(\tau)$ и $\pi(y, v, \tau)$ для $\tau>0$.

Чтобы специфицировать начальные условия, мы положим $\pi(y, v, 0)=\pi_{0}(y, v)$ и $Y(0)=0.5$. Начальную скорость $W(0)$ специфицировать не нужно, она определяется "свободно" как решение уравнения (2.7) в момент времени $\tau=0$. Легко проверить, что начальная скорость $|W(0)|$ будет меньше, чем $v_{\min }$, на самом деле, $W(0) \rightarrow 0$ при $\varepsilon_{0} \rightarrow 0$ в (P5).

Сначала мы определим условия, при которых уравнение (2.7) имеет решение $W$. Пусть

$$
v_{\sup }^{-}(\tau)=\sup \{v: \pi(Y-0, v, \tau)>0\}
$$

(считаем, что супремум пустого множества есть $-\infty$ ) и

$$
v_{\text {inf }}^{+}(\tau)=\inf \{v: \pi(Y+0, v, \tau)>0\}
$$

(аналогично, инфимум пустого множества полагается равньм $+\infty$ ).

ЛЕмма 2.1. Возможнив три случая:

(a) если $v_{\mathrm{sup}}^{-}>v_{\mathrm{inf}}^{+}$или $v_{\mathrm{sup}}^{-}=v_{\mathrm{inf}}^{+} \in \mathbb{R}$, mо (2.7) имеет единственное решение $W \in\left[v_{\text {inf }}^{+}, v_{\text {sup }}^{-}\right]$

(b) если $v_{\mathrm{sup}}^{-}<v_{\mathrm{inf}}^{+}$, то решения (2.7) занимают цельй отрезок $\left[v_{\mathrm{sup}}^{-}, v_{\mathrm{inf}}^{+}\right]$;

(c) если $v_{\text {sup }}^{-}=v_{\text {inf }}^{+}=\infty$ или $v_{\text {sup }}^{-}=v_{\text {inf }}^{+}=-\infty$, то (2.7) не имеет действительных решений.

ДокАЗАТЕЛЬСТво. В случае (а) разность между левой и правой частями в (2.7) является непрерьвной и строго монотонно убываюшей функцией от $W$. При $W<$ $v_{\text {inf }}^{+}$эта разность положительна, а для $W>v_{\text {sup }}^{-}$- отрицательна. Оставшаяся часть доказательства проводится прямой проверкой.

Легко показать (мы не будем этого делать), что при наших предположениях (P1)$(\mathrm{P} 4)$ для каждого $\tau>0$ плотность $\pi(y, v, \tau)$ имеет компактный носитель в $(y, v)$-плоскости, т.е. $\pi(y, v, \tau) \equiv 0$ для всех $|v|>v_{\max }(\tau)$. Следовательно, случаю (с) "отсутствия решения" никогда нет места. Случай (b) многих решений очень маловероятен, но не невозможен. Если он происходит, то скорость $W(\tau)$ должна быть определена однозначно с помошью дополнительного требования:

$\left(\mathrm{H}^{\prime}\right)$ Если $W(\tau-0) \in\left[v_{\mathrm{sup}}^{-}, v_{\mathrm{inf}}^{+}\right]$, мы определяем $W(\tau)$ по непрерьвности: $W(\tau)=$ $W(\tau-0)$. Если $W(\tau-0)<v_{\text {sup }}^{-}$или $W(\tau-0)>v_{\text {inf }}^{+}$, мыполагаем $W(\tau)=v_{\text {sup }}^{-}$ или $W(\tau)=v_{\text {inf }}^{+}$соответственно.

Это завершает определение $W(\tau)$, начатое в $(\mathrm{H} 4)$.

Для кусочно гладких плотностей $\pi(y, v, \tau)$ общего положения скорость $W(\tau)$ непрерьвна, но в некоторых случаях ее непрерьвность может быть нарушена. Однако следуюшая простая лемма будет полезной. 
Лемма 2.2. Предположим, ито для каждого $\tau \in[a, b]$ плотность $\pi(y, v, \tau)$ является кусочно $C^{1}$-функиией и имеет конечное число $C^{1}$-гладких линий разрыва на $(y, v)$-плоскости с положительными наклонами, как мы требовали от $\pi_{0}(y, v)$ в $\S 1$. Тогда $W(\tau)$ будет непрерывна и кусочно дифференцируема на отрезке $[a, b]$.

Теперь мы прерываем изложение, чтобы сделать несколько замечаний. Масса поршня никогда не используется в наших уравнениях, потому что его макроскопическая масса равна нулю. В самом деле, для механической системы, описанной в $\S 1$, масса поршня $\sim L^{2}$, в то время как масса частиц газа $\sim L^{3}$; следовательно, относительная масса поршня исчезает при $L \rightarrow \infty$. Рассмотрим теперь обшую (макроскопическую) массу газа

$$
\mathscr{M}_{\mathrm{tot}}(\tau)=\int_{0}^{1} \int \pi(y, v, \tau) d v d y
$$

и отдельно массы в левой и правой частях

$$
\begin{aligned}
& \mathscr{M}_{L}(\tau)=\int_{0}^{Y(\tau)} \int \pi(y, v, \tau) d v d y \\
& \mathscr{M}_{R}(\tau)=\int_{Y(\tau)}^{1} \int \pi(y, v, \tau) d v d y
\end{aligned}
$$

и общую кинетическую энергию

$$
2 \mathscr{E}_{\mathrm{tot}}(\tau)=\int_{0}^{1} \int v^{2} \pi(y, v, \tau) d v d y
$$

Следуюшую лемму мы оставляем читателю в качестве (простого) упражнения.

Лемма 2.3. Величины $\mathscr{M}_{\text {tot }}, \mathscr{M}_{L}, \mathscr{M}_{R}$ и $\mathscr{E}_{\text {tot }}$ остаются постоянныцми при всех $\tau$. Основное уравнение (2.7) сохраняет также обший импульс $\iint v \pi(y, v, \tau) d v d y$, но
эта величина меняется при столкновениях со стенками.

ЗАмечАние. Ранее Лебовиц, Пясецкий и Синай [19] изучали динамику поршня, по сушеству, при тех же самых начальных условиях, что и наши (P1)-(P5). Они на эвристическом уровне объяснили, что динамика поршня может быть приближена некоторыми детерминистскими уравнениями в начальных (микроскопических) переменных $x$ и $t$. В действительности, настояшая работа возникла как продолжение [19]. Детерминистские уравнения в [19] соответствуют нашим (2.2)-(2.6) с очевидньм преобразованием назад к переменным $x, t$, но наше основное уравнение $(2.7)$ отлично в контексте [19] и имеет вид:

$$
\frac{d}{d t} V(t)=a\left[\int_{V}^{\infty}(v-V(t))^{2} \pi(Y-0, v, t) d v-\int_{-\infty}^{V}(v-V(t))^{2} \pi(Y+0, v, t) d v\right] .
$$


Здесь $X=X(t)$ и $V=V(t)=\dot{X}(t)$ обозначают детерминистские положение и скорость поршня, а $\pi(x, v, t)$-плотность газа (постоянная $a$ появляется в (1.4)). Мы отсылаем читателя к [19] за деталями и эвристическим вьводом уравнения (2.9). Поскольку уравнение уравнение (2.9), в отличие от нашего (2.7), является дифференциальньм уравнением, начальная скорость $V(0)$ должна быть задана отдельно; принято полагать $V(0)=0$. Уравнение (2.9) может быть сведено к (2.7) в пределе при $L \rightarrow \infty$ следуюшим образом. Можно показать (мы опускаем детали), что (2.9) есть диссипативное уравнение, решение которого с любыми (достаточно мальми) начальными условиями $V(0)$ сходится к решению уравнения $(2.7)$ за временной $t$-интервал $\sim \ln L$. Этот интервал имеет длину $\sim L^{-1} \ln L$ на оси $\tau$, и, таким образом, он обрашается в нуль при $L \rightarrow \infty$. Этим объясняется, почему мы заменяем (2.9) уравнением (2.7) и игнорируем начальное условие $V(0)$, когда работаем с термодинамическими переменными $\tau$ и $y$. По тем же причинам будет удобно заменить начальное значение скорости поршня $V(0)=0$ в механической модели $\S 1$ на $V(0)=W(0)$, см. теорему 3.5 ниже. Уравнение (2.9) не будет использоваться дальше в этой статье.

Мы теперь опишем более детально решение гидродинамических уравнений (H1)(H4). Предположим, что для некоторого $\tau>0$ плотность газа $\pi(y, v, \tau)$ удовлетворяет следуюшим требованиям, подобньм (P1)-(Р5), которые были наложены на начальную функцию в $\S 1$.

$\left(\mathrm{P}^{\prime}\right)$ Гладкость. $\pi(y, v, \tau)$ - кусочно $C^{1}$-функция с равномерно ограниченными частными производньми, т.е. $|\partial \pi / \partial y| \leqslant D_{1}^{\prime}$ и $|\partial \pi / \partial v| \leqslant D_{1}^{\prime}$ для некоторого $D_{1}^{\prime}>0$.

$\left(\mathrm{P} 2^{\prime}\right)$ Линии разрыва. $\pi(y, v, \tau)$ имеет конечное число $\left(\leqslant K_{1}^{\prime}\right)$ линий разрыва на $(y, v)$-плоскости со строго положительными наклонами (каждая линия задается уравнением $v=f(y)$, где $f(y)$ - функция класса $C^{1}$ и $0<c_{1}^{\prime}<f^{\prime}(y)<$ $\left.c_{2}^{\prime}<\infty\right)$.

(P3') Границы плотности. Пусть

$$
\pi(y, v, \tau)>\pi_{\min }^{\prime}>0 \text { при } v_{1}^{\prime}<|v|<v_{2}^{\prime}
$$

для некоторых $0<v_{1}^{\prime}<v_{2}^{\prime}<\infty$ и

$$
\sup _{y, v} \pi(y, v, \tau)=\pi_{\max }^{\prime}<\infty .
$$

(P4') "Обрезание скорости". Пусть

$$
\pi(y, v, \tau)=0 \text { при }|v| \leqslant v_{\min }^{\prime} \text { или }|v| \geqslant v_{\max }^{\prime}
$$

$$
\text { для некоторых } 0<v_{\min }^{\prime}<v_{\max }^{\prime}<\infty \text {. }
$$

Наконец, мы хотим предположить, подобно (Р5), что $\pi(y, v, \tau)$ почти симметрична около поршня, но это предположение требует небольшой дополнительной работы, так как поршень не обязан оставаться в средней точке $Y(0)=0.5$. Для каждого $Y \in(0,1)$ обозначим через $h_{Y}$ единственньй гомеоморфизм отрезка $[0,1]$ такой, что $h_{Y}(0)=1$, $h_{Y}(1)=0, h_{Y}(Y)=Y$ и $h_{Y}$ линеен на сегментах $[0, Y]$ и $[Y, 1]$. Затем мы рассмотрим $[0, Y] \times \mathbb{R}$ как многообразие, в котором точки $(0, v)$ и $(0,-v)$ отождествлены для всех $v>0$, и таким же образом поступим с точками $(Y, v)$ и $(Y,-v)$ для всех $v>0$. 
Аналогично, на многообразии $[Y, 1] \times \mathbb{R}$ отождествим точки $(1, v)$ и $(1,-v)$, а также точки $(Y, v)$ и $(Y,-v)$ для всех $v>0$. Мы обозначим $d_{Y}$ расстояние на каждом из этих двух многообразий, индуцированное евклидовой метрикой $\left(d y^{2}+d v^{2}\right)^{1 / 2}$. Причина, по которой мы нуждаемся в этом специальном расстоянии, станет ясна позже, в доказательстве предложения 2.10 .

(P5') Приближенное равенство давлений. Мы потребуем, чтобы для достаточно малого $\varepsilon_{0}^{\prime}>0$

$$
|Y(\tau)-0.5|<\varepsilon_{0}^{\prime}
$$

и для любой точки $(y, v)$ с $0 \leqslant y \leqslant 1$ и $v_{\min }^{\prime} \leqslant|v| \leqslant v_{\max }^{\prime}$ существовала другая точка $\left(y_{*}, v_{*}\right)$ "за поршнем" (т.е. такая, что $(y-Y)\left(y_{*}-Y\right)<0$, где $\left.Y=Y(\tau)\right)$, удовлетворяющая неравенствам

$$
d_{Y}\left(\left(y_{*}, v_{*}\right),\left(h_{Y}(y),-v\right)\right)<\varepsilon_{0}^{\prime}
$$

и

$$
\left|\pi(y, v, \tau)-\pi\left(y_{*}, v_{*}, \tau\right)\right|<\varepsilon_{0}^{\prime} .
$$

Кроме того, мы потребуем, чтобы было выполнено неравенство

$$
\varepsilon_{0}^{\prime}<C_{0}^{\prime} \varepsilon_{0}
$$

с некоторой постоянной $C_{0}^{\prime}>0$.

В действительности, отображение $(y, v) \mapsto\left(y_{*}, v_{*}\right)$, введенное в $\left(\mathrm{P} 5^{\prime}\right)$, которое мы будем обозначать через $R_{\tau}$, является взаимно однозначньм и будет явно построено ниже в доказательстве предложения 2.10 .

Мы опять рассматриваем $D_{1}^{\prime}, K_{1}^{\prime}, c_{1}^{\prime}, c_{2}^{\prime}, v_{1}^{\prime}, v_{2}^{\prime}, v_{\min }^{\prime}, v_{\max }^{\prime}, \pi_{\min }^{\prime}, \pi_{\max }^{\prime}$, а также $C_{0}^{\prime}$ как глобальные константы. Они должны быть ограничены на том временном интервале, на котором мы рассматриваем динамику (a $v_{\min }^{\prime}, \pi_{\min }^{\prime}$ должны быть отделены от нуля); значит, мы можем обрашаться с этими константами, как с не зависяшими от $\tau$. Согласно (2.16) $\varepsilon_{0}^{\prime}$, точно так же, как $\varepsilon_{0}$ в (Р5), есть дополнительный малый параметр.

Теперь мы получим достаточно элементарные, но важные следствия из указанных вьше предположений. Так как плотность $\pi(y, v, \tau)$ обрашается в нуль при $|v|<v_{\min }^{\prime}$, так же ведет себя и функция $q(v, \tau ; Y, W)$, определенная в (2.8). Кроме того, для всех $|W|<v_{\min }^{\prime}$ функция $q(v, \tau ; Y, W)$ не будет зависеть от $W$ и может быть переопределена следуюшим образом:

$$
q(v, \tau ; Y)= \begin{cases}\pi(Y+0, v, \tau) & \text { при } v<0 \\ \pi(Y-0, v, \tau) & \text { при } v>0 .\end{cases}
$$

$\mathrm{K}$ тому же уравнение (2.7) может быть упрощено: $\operatorname{sign}(v-W)$ может быть заменен на $\operatorname{sign} v$. Тогда раскрытие квадрата в (2.7) сводит его к обычному квадратному уравнению для $W$ :

$$
\mathscr{Q}_{0} W^{2}-2 \mathscr{Q}_{1} W+\mathscr{Q}_{2}=0,
$$


где

$$
\begin{aligned}
& \mathscr{Q}_{0}=\int \operatorname{sign} v \cdot q(v, \tau ; Y) d v, \\
& \mathscr{Q}_{1}=\int v \operatorname{sign} v \cdot q(v, \tau ; Y) d v, \\
& \mathscr{Q}_{2}=\int v^{2} \operatorname{sign} v \cdot q(v, \tau ; Y) d v
\end{aligned}
$$

с $Y=Y(\tau)$. Интегралы $\mathscr{Q}_{0}, \mathscr{Q}_{1}, \mathscr{Q}_{2}$ имеют следующий физический смысл:

$$
\begin{aligned}
& m \mathscr{Q}_{0}=m_{L}-m_{R}, \\
& m \mathscr{Q}_{1}=p_{L}-p_{R}, \\
& m \mathscr{Q}_{2}=2\left(e_{L}-e_{R}\right),
\end{aligned}
$$

где $m_{L}, p_{L}, e_{L}$ представляют собой обшую массу, импульс и энергию входяших частиц газа (на единицу длины) с левой стороны от поршня, а $m_{R}, p_{R}, e_{R}$ - такие же величины с правой стороны от него. Значение $\mathscr{Q}_{2}$ также представляет собой обшее давление, оказьваемое на поршень газом, если поршень не двигается. Конечно, если $\mathscr{Q}_{2}(\tau)=0$, мы должны иметь $W(\tau)=0$, что согласуется с (2.18). Приводимая далее лемма легко следует из $\left(\mathrm{P}^{\prime}\right)-\left(\mathrm{P} 5^{\prime}\right)$. Она означает, что функция $q(v, \tau ; Y(\tau))$ почти симметрична по $v$ около 0 .

ЛЕмма 2.4. Для любой определенной для $v>0$ гладкой функиии $f(v)$

$$
\left|\int_{0}^{\infty} f(v) q(v, \tau ; Y(\tau)) d v-\int_{-\infty}^{0} f(-v) q(v, \tau ; Y(\tau)) d v\right| \leqslant C_{f} \varepsilon_{0}
$$

где множитель $C_{f}>0$ зависит от $f$, но не от $\varepsilon_{0}$.

СоглАшениЕ. Мы назьваем постоянные, не зависяшие от нашего дополнительного малого параметра $\varepsilon_{0}$ в $(\mathrm{P} 5)$ или $\left(\mathrm{P} 5^{\prime}\right)$, глобальными постоянными (как $C_{f}$ в предыдушей лемме). Все постоянные в (P1)-(P5) и (P1')-(P5') являются глобальными, за исключением $\varepsilon_{0}$ и связанной с ней $\varepsilon_{0}^{\prime}$. Во многих случаях мы будем обозначать различные глобальные постоянные через $C_{i}, i \geqslant 0$, или просто через $C$.

Из леммы 2.4 вытекает, что $\mathscr{Q}_{0}$ и $\mathscr{Q}_{2}$ малы, более точно,

$$
\max \left\{\left|\mathscr{Q}_{0}\right|,\left|\mathscr{Q}_{2}\right|\right\} \leqslant C \varepsilon_{0},
$$

где $C>0$ - глобальная постоянная. В то же самое время предположение $\left(\mathrm{P}^{\prime}\right)$ гарантирует, что

$$
\mathscr{Q}_{1} \geqslant \mathscr{Q}_{1, \min }>0
$$

для другой глобальной постоянной $\mathscr{Q}_{1, \mathrm{~min}}$.

Если $\varepsilon_{0}$ достаточно мало, то существует единственньй корень квадратичного многочлена $(2.18)$ на интервале $\left(-v_{\min }^{\prime}, v_{\min }^{\prime}\right)$, который соответствует единственному решению уравнения (2.7). Так как этот корень меньше по абсолютной величине другого корня уравнения (2.18), то его можно выразить по формуле

$$
W=\frac{\mathscr{Q}_{1}-\sqrt{\mathscr{Q}_{1}^{2}-\mathscr{Q}_{0} \mathscr{Q}_{2}}}{\mathscr{Q}_{0}},
$$


где знак перед корнем - , а не + . Конечно, $(2.24)$ применимо, когда $\mathscr{Q}_{0} \neq 0$; при $\mathscr{Q}_{0}=0$ мы просто имеем

$$
W=\frac{\mathscr{Q}_{2}}{2 \mathscr{Q}_{1}} .
$$

СЛЕДСТВИЕ 2.5. Если $\varepsilon_{0}$ достаточно мало, то

$$
|W(\tau)| \leqslant \mathscr{B} \varepsilon_{0}<v_{\min }^{\prime} / 3
$$

для некоторой глобальной постоянной $\mathscr{B}>0$.

ДокАЗАТЕльство. Следует немедленно из соотношений (2.22)-(2.25).

Теперь мы сделаем важное замечание.

ЗАмечАНИЕ (о продолжении). Рассмотрим плотность входящих частиц газа слева от поршня, т.е. $\pi(y, v, \tau)$ для $y=Y(\tau)-0$ и $v>v_{\min }^{\prime}$. Эта функция "оканчивается" на поршне, т.е. имеет разрьв по $y$ при $y=Y(\tau)$. Но она может быть продолжена естественно гладким образом “через поршень" , т.е. для $y>Y(\tau)$, если проигнорировать взаимодействие газа, приходяшего слева, с поршнем на временах $s \in(\tau-\delta, \tau)$ и применить взамен правило (Н1), как если бы газ “прошел через поршень". Это определяет гладкое продолжение функции $\pi(y, v, \tau)$ из области $y \leqslant Y(\tau)$ на область $Y(\tau)<y<Y(\tau)+O(\delta)$ для всех $v \geqslant v_{\min }^{\prime}$. Это продолжение позволяет нам дифференцировать $q(v, \tau ; Y)$, определенное с помощью (2.17), по $Y$ для любых $v \geqslant v_{\min }^{\prime}$. Аналогичное продолжение может быть сделано для плотности $\pi(y, v, \tau)$ из области $y \geqslant Y(\tau)$ на область $Y(\tau)-O(\delta)<y<Y(\tau)$ для всех $v \leqslant-v_{\min }^{\prime}$; значит, $q(v, \tau ; Y)$ становится дифференцируемой по $Y$ для $v \leqslant-v_{\min }^{\prime}$. Мы замечаем, что наше продолжение может быть однозначно определено, поскольку оно нужно нам только для $|v| \geqslant v_{\min }^{\prime}$, в то время как скорость поршня остается меньше, чем $v_{\min }^{\prime}$.

Теперь величины $\mathscr{Q}_{0}, \mathscr{Q}_{1}$ и $\mathscr{Q}_{2}$, определенные в $(2.19)-(2.21)$, становятся дифференцируемыми по $Y$ для каждого фиксированного $\tau$, и из предположений $\left(\mathrm{P} 1^{\prime}\right)-\left(\mathrm{P} 4^{\prime}\right)$ легко следует, что

$$
\left|d \mathscr{Q}_{i} / d Y\right| \leqslant C_{1}, \quad i=0,1,2,
$$

где $C_{1}>0$ - глобальная постоянная.

СлЕДСТВИЕ 2.6. Ускорение поршня $A(\tau)=d W(\tau) / d \tau$ удовлетворяет неравенству

$$
|A(\tau)| \leqslant C \varepsilon_{0}
$$

с некоторой глобальной постоянной $C>0$.

ДокАЗАТЕльство. Дифференцируя квадратное уравнение (2.18) по $\tau$, мы получим

$$
A(\tau)=\frac{\left(d \mathscr{Q}_{0} / d \tau\right) W^{2}-2\left(d \mathscr{Q}_{1} / d \tau\right) W+\left(d \mathscr{Q}_{2} / d \tau\right)}{2\left(\mathscr{Q}_{1}-\mathscr{Q}_{0} W\right)} .
$$

Ясно, что знаменатель этой дроби отделен от нуля, а числитель имеет верхнюю границу порядка $\varepsilon_{0}$, поскльку $\left|d \mathscr{Q}_{i} / d \tau\right|=\left|\left(d \mathscr{Q}_{i} / d Y\right) W\right| \leqslant \mathrm{const} \cdot \varepsilon_{0}$, как следует из (2.27) и (2.26). Следствие доказано.

Более важно, что теперь мы можем доказьвать существование и единственность решения гидродинамических уравнений (H1)-(H4) до тех пор, пока условия (P1')$\left(\mathrm{P} 5^{\prime}\right)$ продолжают вьполняться: 
ЛЕмма 2.7. Если гидродинамические уравнения (H1)-(H4) имеют решения на интервале $0 \leqslant \tau \leqslant T$ и условия $\left(\mathrm{P}^{\prime}\right)-\left(\mathrm{P} 5^{\prime}\right)$ выполнены на этом интервале, тогда решение единственно в момент $\tau=T$ и может быть продолжено непосредственно за точку $\tau=T$.

ДокАЗАТЕльство. Только уравнение (2.6) является дифференциальньм в нашей системе $(\mathrm{H} 1)-(\mathrm{H} 4)$ и в нем $W(\tau)$ определяется по формулам $(2.24),(2.25)$ как корень квадратного уравнения (2.18). Ввиду замечания о продолжении (см. вьше) мы можем рассматривать $W$ как неявную функцию от $Y$, т.е. на самом деле $W=F(Y, \tau)$. Тогда дифференциальное уравнение (2.6) принимает канонический вид

$$
\frac{d}{d \tau} Y(\tau)=F(Y(\tau), \tau)
$$

Для того чтобы это уравнение имело единственное решение, достаточно, чтобы $F(Y, \tau)$ имела ограниченную частную производную по $Y$.

Так как $W$ - корень квадратного уравнения (2.18), мы получим, дифференцируя его по $Y$, что

$$
\frac{\partial F(Y, \tau)}{\partial Y}=\frac{\left(d \mathscr{Q}_{0} / d Y\right) W^{2}-2\left(d \mathscr{Q}_{1} / d Y\right) W+\left(d \mathscr{Q}_{2} / d Y\right)}{2\left(\mathscr{Q}_{1}-\mathscr{Q}_{0} W\right)} .
$$

Мы уже знаем, что знаменатель отделен от нуля. Из (2.27) следует, что числитель остается ограниченным сверху; поэтому

$$
\left|\frac{\partial F(Y, \tau)}{\partial Y}\right| \leqslant \kappa
$$

для некоторой глобальной постоянной $\kappa>0$. Лемма доказана.

Рассмотрим теперь эволюцию точки $(y, v)$ в области

$$
\mathscr{G}:=\{(y, v): 0 \leqslant y \leqslant 1\}
$$

происходящую по правилам (Н1)-(Н3) (свободное движение с постоянной скоростью и упругие столкновения со стенками и поршнем). Обозначим $\left(y_{\tau}, v_{\tau}\right)$ положение и скорость этой точки при $\tau \geqslant 0$. Тогда (H1) переходит в $\dot{y}_{\tau}=v_{\tau}$ и $\dot{v}_{\tau}=0$ при $y_{\tau} \notin\{0,1, Y(\tau)\},(\mathrm{H} 2)$ переходит в $\left(y_{\tau+0}, v_{\tau+0}\right)=\left(y_{\tau-0},-v_{\tau-0}\right)$ при $y_{\tau-0} \in\{0,1\}, \mathrm{a}$ (H3) - в

$$
\left(y_{\tau+0}, v_{\tau+0}\right)=\left(y_{\tau-0}, 2 W(\tau)-v_{\tau-0}\right)
$$

при $y_{\tau-0}=Y(\tau)$. Отметим, что (2.31) соответствует специальному случаю законов механического столкновения (1.2)-(1.3) с $\varepsilon=0$ (эквивалентно, $m=0$ ). Поэтому точка $(y, v)$ движется в $\mathscr{G}$, как если бы она представляла собой частицу газа с нулевой массой.

Движение точки $(y, v)$ описьвается однопараметрическим семейством преобразований $\mathscr{F}^{\tau}: \mathscr{G} \rightarrow \mathscr{G}$, заданных формулой $\mathscr{F}^{\tau}\left(y_{0}, v_{0}\right)=\left(y_{\tau}, v_{\tau}\right)$ для $\tau>0$. Мы будем также писать, что $\mathscr{F}^{-\tau}\left(y_{\tau}, v_{\tau}\right)=\left(y_{0}, v_{0}\right)$. Согласно $(\mathrm{H} 1)-(\mathrm{H} 3)$ плотность $\pi(y, v, \tau)$ удовлетворяет простому уравнению

$$
\pi\left(y_{\tau}, v_{\tau}, \tau\right)=\pi\left(\mathscr{F}^{-\tau}\left(y_{\tau}, v_{\tau}\right), 0\right)=\pi_{0}\left(y_{0}, v_{0}\right)
$$


для всех $\tau>0$. Легко видеть, что для каждого $\tau>0$ отображение $\mathscr{F}^{\tau}$ взаимно однозначно и сохраняет площадь, т.е. $\operatorname{det}\left|D \mathscr{F}^{\tau}(y, v)\right|=1$.

Далее, ввиду $(\mathrm{P} 4)$ начальная плотность $\pi_{0}(y, v)$ может быть положительной только в области

$$
\mathscr{G}^{+}:=\left\{(y, v): 0 \leqslant y \leqslant 1, v_{\min } \leqslant|v| \leqslant v_{\max }\right\} .
$$

Поэтому мы ограничимся рассмотрением точек $(y, v) \in \mathscr{G}^{+}$. Для любого $\tau>0$ образы этих точек будут заключены в область $\mathscr{G}^{+}(\tau):=\mathscr{F}^{\tau}\left(\mathscr{G}^{+}\right)$. В частности, $\pi(y, v, \tau)=0$ для $(y, v) \notin \mathscr{G}^{+}(\tau)$.

Мы теперь сделаем важное наблюдение. Если точка $\left(y_{\tau}, v_{\tau}\right)$ столкнулась с поршнем, который движется медленно, $|W(\tau)| \ll\left|v_{\tau}\right|$, то следуюшее столкновение этой точки с поршнем не может произойти слишком скоро: точка должна сначала продвинуться к стенке, отскочить от нее и затем вернуться назад к поршню, прежде чем удариться о него снова. Количественная формулировка этого наблюдения содержится в следуюшей лемме.

ЛЕмма 2.8. Пусть точка $\left(y_{\tau}, v_{\tau}\right) \in \mathscr{G}^{+}(\tau)$ сталкивается с поршнем, т.е. $y_{\tau}=Y(\tau)$. Тогда в интервале $(\tau, \tau+\Delta)$, где

$$
\Delta=\frac{1-2 \mathscr{B} \varepsilon_{0} \tau}{v_{\max }^{\prime}+3 \mathscr{B} \varepsilon_{0}}
$$

она не мохсет снова столкнуться с поршнем, т.е. $y_{s} \neq Y(s)$ для $s \in(\tau, \tau+\Delta)$ в предположсении, что $\left(\mathrm{P} 1^{\prime}\right)-\left(\mathrm{P} 5^{\prime}\right)$ выполняются в этом интервале.

ДокАЗАТЕЛьство. Скорость точки после столкновения самое меньшее $v_{\min }^{\prime}-$ $2 \mathscr{B} \varepsilon_{0}$ и самое большее $v_{\max }^{\prime}+2 \mathscr{B} \varepsilon_{0}$. С такой скоростью поршень не может догнать точку, так как $|W(\tau)|<v_{\min }^{\prime}-2 \mathscr{B} \varepsilon_{0}$ согласно (2.26). Итак, точка движется к стенке, отскакивает от нее и движется назад к поршню, и все это занимает время

$$
\Delta \geqslant 2 D /\left(v_{\max }^{\prime}+3 \mathscr{B} \varepsilon_{0}\right)
$$

где $D=\min \{Y(\tau), 1-Y(\tau)\} \geqslant 0.5-\mathscr{B} \varepsilon_{0} \tau$. Лемма доказана.

Таким образом, пока $\left(\mathrm{P} 1^{\prime}\right)-\left(\mathrm{P} 4^{\prime}\right)$ выполнены, столкновения каждой движушейся точки $\left(y_{\tau}, v_{\tau}\right) \in \mathscr{G}^{+}(\tau)$ с поршнем происходят в хорошо разделенные моменты времени, что позволяет нам эффективно их считать. Пусть для $(x, v) \in \mathscr{G}^{+}$

$$
N(y, v, \tau)=\#\left\{s \in(0, \tau): y_{s}=Y(s), v_{s} \neq W(s)\right\}
$$

- число столкновений точки $(y, v)$ с поршнем в интервале $(0, \tau)$. Для каждого $\tau>0$ мы разбиваем область $\mathscr{G}^{+}(\tau)$ на подобласти

$$
\mathscr{G}_{n}^{+}(\tau):=\left\{\mathscr{F}^{\tau}(y, v):(y, v) \in \mathscr{G}^{+} \& N(y, v, \tau)=n\right\}
$$

так что $\mathscr{G}_{n}^{+}(\tau)$ занято точками, испытавшими за интервал времени $(0, \tau)$ в точности $n$ столкновений с поршнем. 
Для каждого $n \geqslant 1$ мы определяем $\tau_{n}>0$ как первый момент времени, в которьй точка $\left(y_{\tau}, v_{\tau}\right) \in \mathscr{G}^{+}(\tau)$ сталкивается с поршнем в $(n+1)$-й раз, т.е.

$$
\tau_{n}=\sup \left\{\tau>0: \mathscr{G}_{n+1}^{+}(\tau)=\varnothing\right\}
$$

В частности, $\tau_{1}>0$ есть самый ранний момент времени, в которьй какая-либо точка $\left(y_{\tau}, v_{\tau}\right) \in \mathscr{G}^{+}(\tau)$ испытьвает свое первое перестолкновение с поршнем. Следовательно, никаких перестолкновений не происходит на интервале $\left[0, \tau_{1}\right)$, и мы назьваем его интервалом без перестолкновений. Аналогично, на интервале $\left(\tau_{1}, \tau_{2}\right)$ для каждой точки возможно не более одного перестолкновения с поршнем, и мы назьваем этот интервал интервалом одного перестолкновения.

Момент времени $\tau_{*}$, указанньй в теореме 1.2 , есть самое раннее время, когда точка $\left(y_{\tau}, v_{\tau}\right) \in \mathscr{G}^{+}(\tau)$ испытьвает или третье столкновение с поршнем, или второе столкновение с поршнем при условии, что первое столкновение произошло после момента времени $\tau_{1}$. Следовательно, $\tau_{*} \leqslant \tau_{2}$ и $\tau_{*}$, в действительности, очень близко к $\tau_{2}$, как видно из следующей леммы.

Лемма 2.9. Пусть $\left(\mathrm{P} 1^{\prime}\right)-\left(\mathrm{P} 5^{\prime}\right)$ выполнены на интервале $\left(0, n / v_{\max }+\delta\right)$ для некоторых $n \geqslant 1$ и $\delta>0$. Тогда для всех достаточно мальх $\varepsilon_{0}$

$$
\left|\tau_{k}-k / v_{\max }\right| \leqslant C \varepsilon_{0}
$$

для всех $1 \leqslant k \leqslant n$, где $C>0$ - глобальная постоянная, которая может зависеть от $n$. K тому же

$$
\left|\tau_{*}-2 / v_{\max }\right| \leqslant C \varepsilon_{0} .
$$

ДокАЗАТЕльство. Необходимые нижние границы для $\tau_{k}$ следуют из леммы 2.8 . Необходимые верхние границы получаются так же легко; мы опускаем детали.

Ясно, что гидродинамические уравнения (H1)-(H4) будут иметь единственное и "хорошее" решение, пока условия $\left(\mathrm{P} 1^{\prime}\right)-\left(\mathrm{P} 5^{\prime}\right)$ продолжают вьполняться с некоторьм мальм $\varepsilon_{0}$. Наша следуюшая цель - показать, что эти условия действительно вьполняются.

ПРЕДЛОЖЕНИЕ 2.10. Пусть $Т>0$. Если начальная плотность $\pi_{0}(y, v)$ удовлетворяет условиям (P1)-(P5) и $\varepsilon_{0}$ в (P5) достаточно мало (для данного T), то условия $\left(\mathrm{P}^{\prime}\right)-\left(\mathrm{P}^{\prime}\right)$ будут выполнены в интервале $0<\tau<T$.

ЗАмечАниЕ. Соответствуюшие глобальные постоянные в $\left(\mathrm{P} 1^{\prime}\right)-\left(\mathrm{P} 5^{\prime}\right)$ будут зависеть от $T$, как объясняется далее.

ДокАЗАТЕльство. Основная идея заключается в том, чтобы показать, что ограничения, наложенные на $\pi(y, v, \tau)$ в (P1)-(P5) при $\tau=0$, “ухудшаются" со временем очень медленно, так что (P1')-(P5') будут вьполняться ("распространяться") с соответствуюшими глобальными постоянными, медленно меняюшимися во времени.

Сначала мы отметим, что пока $\left(\mathrm{P} 1^{\prime}\right)-\left(\mathrm{P} 5^{\prime}\right)$ вьполнены, число столкновений растет по $\tau$ не быстрее чем линейно, т.е. на любом интервале $(0, \tau)$, на котором $\left(\mathrm{P} 1^{\prime}\right)-\left(\mathrm{P} 5^{\prime}\right)$ вьполнены, каждая движушаяся точка $\left(y_{s}, v_{s}\right) \in \mathscr{G}^{+}(s)$ испытьвает самое большее 
$\tau v_{\max }^{\prime}+1$ столкновений с поршнем при достаточно малом $\varepsilon_{0}$, см. леммы 2.8 и 2.9 . Далее, мы проверяем каждое из условий $\left(\mathrm{P} 1^{\prime}\right)-\left(\mathrm{P} 5^{\prime}\right)$ в отдельности и показываем, что каждое из них должно вьполняться вплоть до времени $T$ в предположении, что другие вьполнены.

Мы начинаем с (P1). Ввиду (Н1) мы имеем

$$
\frac{\partial \pi(y, v, \tau+s)}{\partial y}=\frac{\partial \pi(y-s v, v, \tau)}{\partial y}
$$

и

$$
\frac{\partial \pi(y, v, \tau+s)}{\partial v}=\frac{\partial \pi(y-s v, v, t)}{\partial v}-s \frac{\partial \pi(y-s v, v, \tau)}{\partial y}
$$

для $s>0$ таких, что движушаяся точка, расположенная в $(y, v)$ в момент времени $\tau+s$, не испытьвала столкновений с поршнем на интервале $(\tau, \tau+s)$. Итак, между столкновениями со стенками и поршнем частные производные от $\pi(y, v, \tau)$ могут расти по $\tau$ не быстрее чем линейно. Столкновения со стенками могут изменить только знак производных от $\pi$, но не их абсолютные значения.

Теперь рассмотрим эффект взаимодействия с поршнем. Мы оцениваем частные производные от $\pi(y, v, \tau)$ в точке $(y, v)$ после столкновения с поршнем в некоторый более ранний момент $s \in(0, \tau)$. Для простоты предположим, что никаких других столкновений движушейся точки $(y, v)$ с поршнем и стенками на интервале $(s, \tau)$ не происходит. Тогда $s$ удовлетворяет уравнению

$$
Y(s)=y-(\tau-s) v .
$$

Из (Н3) и (Н1) мы имеем

$$
\begin{aligned}
\pi(y, v, \tau) & =\pi(y-(\tau-s) v, v, s+0)=\pi(y-(\tau-s) v, 2 W-v, s-0) \\
& =\pi\left(y-(\tau-s) v-\left(s-s_{0}\right)(2 W-v), 2 W-v, s_{0}\right),
\end{aligned}
$$

где $s_{0}<s$ - произвольный более ранний момент времени (который мы считаем фиксированным), а $W=W(s)$ - скорость поршня во время столкновения. Положим $y_{0}=y-(\tau-s) v-\left(s-s_{0}\right)(2 W-v)$ и $v_{0}=2 W-v$. Тогда

$\frac{\partial \pi(y, v, \tau)}{\partial y}=\frac{\partial \pi\left(y_{0}, v_{0}, s_{0}\right)}{\partial y}\left[1+v \frac{d s}{d y}-2\left(s-s_{0}\right) \frac{d W}{d y}-(2 W-v) \frac{d s}{d y}\right]+\frac{\partial \pi\left(y_{0}, v_{0}, s_{0}\right)}{\partial v} \cdot 2 \frac{d W}{d y}$.

Дифференцирование (2.33) по у дает

$$
\frac{d Y}{d s} \cdot \frac{d s}{d y}=1+v \frac{d s}{d y}
$$

откуда

$$
\frac{d s}{d y}=\frac{1}{W-v}
$$

Кроме того,

$$
\frac{d W}{d y}=\frac{d W}{d s} \cdot \frac{d s}{d y}=\frac{A}{W-v},
$$


где $A=A(s)$ - ускорение поршня в момент столкновения. Далее, пока $\left(\mathrm{P} 1^{\prime}\right)-\left(\mathrm{P} 5^{\prime}\right)$ вьполнены, $W=O\left(\varepsilon_{0}\right)$ и $A=O\left(\varepsilon_{0}\right)$; следовательно, $d s / d y=-v^{-1}+O\left(\varepsilon_{0}\right)$ и, таким образом,

$$
\frac{\partial \pi(y, v, \tau)}{\partial y}=\frac{\partial \pi\left(y_{0}, v_{0}, s_{0}\right)}{\partial y}+O\left(\varepsilon_{0}\right)
$$

Другими словами, поршень (благодаря его низкой скорости и ускорению) действует почти как стенка, которая только изменяет знак $\partial \pi / \partial y$. Аналогичные вычисления (мы опускаем их) справедливы для частной производной по $v$.

Значит, пока $\left(\mathrm{P} 1^{\prime}\right)-\left(\mathrm{P} 5^{\prime}\right)$ вьполняются, плотность $\pi(y, v, \tau)$ остается кусочно $C^{1}$ функцией и ее частные производные растут по $\tau$ не быстрее чем линейно.

Теперь мы проверим условие $\left(\mathrm{P} 2^{\prime}\right)$. Мы начинаем с трех специальных линий разрыва, которые не появляются явно в (P2). Они возникают немедленно при отражениях от стенок и поршня при $\tau=0$, так как начальная плотность $\pi(y, v, 0)$ не удовлетворяет (H2), (Н3). Эти линии разрыва: $y=0, y=0.5, y=1$ при $\tau=0$, и их образы при $\tau>0$ будут наклонными линиями:

$$
y=v \tau, \quad y=0.5+v \tau, \quad y=1+v \tau
$$

соответственно, см. рис. 2. Таким образом, их наклон в любой момент времени положителен и постоянен: $d y / d v=\tau$. Он не отделен от нуля при $\tau \rightarrow 0$, так что условие $\left(\mathrm{P} 2^{\prime}\right)$ нарушается при малых $\tau$; но станет ясно непосредственно далее, почему это не должно нас беспокоить.

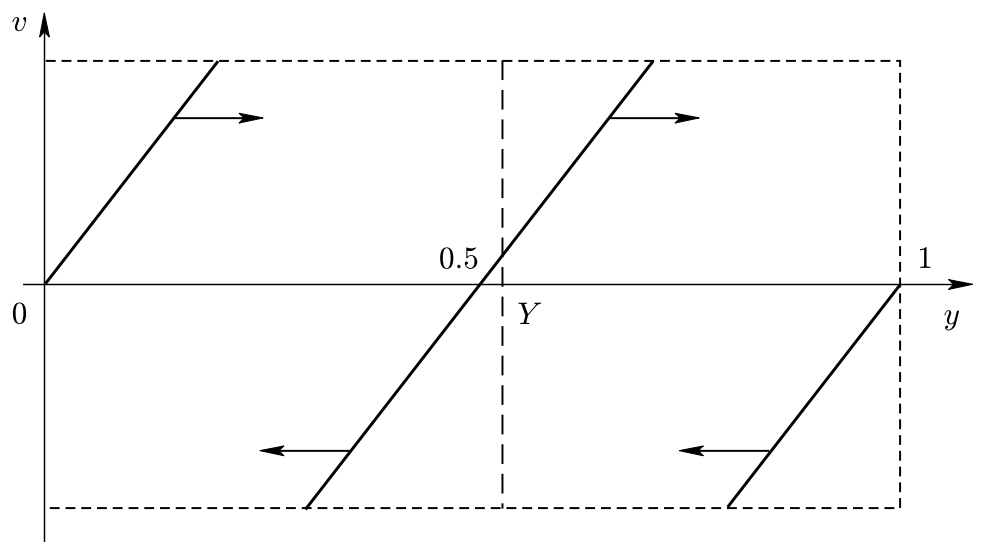

Рис. 2. Наклонные линии разрыва. Пунктирная вертикальная линия показьвает положение поршня

Линии разрьва (2.34) существуют только в области $v_{\min } \leqslant|v| \leqslant v_{\max }$ (когда $p \equiv 0)$, и поэтому они не могут пересекать поршень $y=Y(\tau)$ для малых $\tau$. Должно пройти некоторое время, по крайней мере

$$
\tau^{*}=\frac{0.5}{v_{\max }+\mathscr{B} \varepsilon_{0}}>0,
$$


прежде чем какая-либо из этих сингулярных линий “достигнет” поршня и эффект этого пересечения должен будет проявиться. В это время наклоны этих линий будут отделены от нуля: $d y / d v \geqslant \tau^{*}>0$, и, значит, $\left(\mathrm{P} 2^{\prime}\right)$ будет вьполнено.

Мы рассмотрим теперь эволющию всех кривых разрьва функции $\pi(y, v, \tau)$ при возрастании $\tau$. Пусть кривая разрыва функции $\pi(y, v, s)$ в момент времени $s$ задана уравнением $y=g_{s}(v)$ и ее наклон $h_{s}(v)=d g_{s}(v) / d v$. Так как кривая и ее наклон изменяются со временем, функции $g_{s}$ и $h_{s}$ зависят от $s$. Согласно $(2.2)$ между столкновениями с поршнем и со стенками мы имеем $g_{s+r}(v)=g_{s}(v)+v r$ и, значит,

$$
\frac{d g_{s}(v)}{d s}=v \quad \text { и } \frac{d h_{s}(v)}{d s}=1
$$

Следовательно, между столкновениями с поршнем наклон кривых разрьва растет линейно по $\tau$ (отметим, что, в частности, он остается положительным).

Пусть теперь кривая $y=g_{s}(v)$ пересекает поршень в некоторой точке

$$
g_{s}(v)=Y(s)
$$

(это уравнение определяет $v$ как функцию от $s$ ). После столкновения с поршнем эта точка преобразуется в $(Y, 2 W-v)$ согласно правилу (Н3); здесь $W=W(s)$ - скорость поршня. Если $\tau>s$ фиксировано, то образ нашей точки в момент времени $\tau$ есть $(Y+(\tau-s)(2 W-v), 2 W-v)$. Такие точки образуют кривую на $(y, v)$-плоскости, параметризованную параметром $s$ (временем столкновения). Это будет кривая разрьва плотности $\pi(y, v, \tau)$ в момент времени $\tau$. Пусть $y_{s}=Y+(\tau-s)(2 W-v)$ и $v_{s}=2 W-v$ - координаты точки на этой кривой. Чтобы вычислить наклон $d y_{s} / d v_{s}$ этой кривой, мы сначала продифференцируем $y_{s}$ и $v_{s}$ по параметру $s$ :

$$
\frac{d y_{s}}{d s}=W+(\tau-s)\left[2 \frac{d W}{d s}-\frac{d v}{d s}\right]-(2 W-v)=v-W+(\tau-s)\left[2 A-\frac{d v}{d s}\right]
$$

и

$$
\frac{d v_{s}}{d s}=2 \frac{d W}{d s}-\frac{d v}{d s}=2 A-\frac{d v}{d s}
$$

где $A=A(s)$ - ускорение поршня (в момент столкновения $s$ ). Дифференцирование (2.36) по $s$ и использование (2.35) дает

$$
\frac{d g_{s}(v)}{d s} \cdot \frac{d v}{d s}+v=W
$$

отсюда

$$
\frac{d v}{d s}=\frac{W-v}{h_{s}(v)} .
$$

Поэтому наклон нашей сингулярной кривой в момент времени $\tau$ есть

$$
\frac{d y}{d v}(\tau)=\frac{(v-W)\left[h_{s}(v)+\tau-s\right]+2 A h_{s}(v)(\tau-s)}{v-W+2 A h_{s}(v)} .
$$

При вьполнении $\left(\mathrm{P}^{\prime}\right)-\left(\mathrm{P} 5^{\prime}\right)$ мы имеем $W=O\left(\varepsilon_{0}\right)$ и $A=O\left(\varepsilon_{0}\right)$; тогда

$$
\frac{d y}{d v}(\tau)=h_{s}(v)+\tau-s+O\left(\varepsilon_{0}\right) .
$$

Следовательно, каждое столкновение с поршнем добавляет только поправку порядка $O\left(\varepsilon_{0}\right)$ к линейному росту наклонов кривых разрьва.

Далее мы проверим условия $\left(\mathrm{P} 3^{\prime}\right)-\left(\mathrm{P} 5^{\prime}\right)$, опираясь на следующую лемму. 
Лемма 2.11. Пусть (P1')-(P5') выполнены на интервале $(0, \tau)$. Тогда для каждой точки $(y, v) \in \mathscr{G}^{+}(\tau)$ существует другая точка $\left(y_{0}, v_{0}\right) \in \mathscr{G}^{+}$такая, что $\pi(y, v, \tau)=\pi\left(y_{0}, v_{0}, 0\right) u$

$$
\left\|v|-| v_{0}\right\|=2\left(v_{\max }^{\prime} \tau+1\right) \mathscr{B} \varepsilon_{0} .
$$

ДокАЗАТЕЛЬСТво. Мы полагаем $\left(y_{0}, v_{0}\right)=\mathscr{F}^{-\tau}(y, v)$ и используем $(2.32)$. При каждом столкновении точки $\left(y_{0}, v_{0}\right)$ с поршнем ее скорость $|v|$ изменяется на $2 W \leqslant$ $2 \mathscr{B} \varepsilon_{0}$ согласно $(2.31)$ и $(2.26)$, а число столкновений ограничено числом $v_{\max }^{\prime} \tau+1$. Лемма доказана.

Из леммы 2.11 немедленно следует, что $\left(\mathrm{P}^{\prime}\right)$ и $\left(\mathrm{P} 4^{\prime}\right)$ продолжают вьполняться с глобальньми постоянными $v_{1}^{\prime}, v_{2}^{\prime}, v_{\min }^{\prime}$ и $v_{\max }^{\prime}$, медленно меняюшимися во времени они изменяются самое большее на $C T \varepsilon_{0}$ на интервале $(0, T)$, где $C>0$ - некоторая глобальная постоянная. В частности, $v_{1}^{\prime}$ и $v_{\min }^{\prime}$ остаются положительными, если $\varepsilon_{0}$ достаточно мало. Постоянные $\pi_{\min }^{\prime}$ и $\pi_{\max }^{\prime}$ не меняются вовсе.

Для проверки $\left(\mathrm{P} 5^{\prime}\right)$ мы явно построим отображение $R_{\tau}:(y, v) \mapsto\left(y_{*}, v_{*}\right)$, входящее в (2.14) и (2.15); оно определяется здесь как $R_{\tau}=\mathscr{F}^{\tau} \circ R_{0} \circ \mathscr{F}^{-\tau}$, где $R_{0}(y, v)=$ $(1-y,-v)$ есть простое отражение относительно поршня при $\tau=0$. Теперь $(2.13)$ следует из (2.26) и (2.15) следует из (Р5).

Наконец, мы получим (2.14) из леммы 2.11. Пусть $(y, v)$ - движушаяся точка в момент времени $\tau$ и $\left(y_{0}, v_{0}\right)=\mathscr{F}^{-\tau}(y, v) \in \mathscr{G}^{+}-$ее прообраз в нулевой момент времени. Сравним эволюцию точки $\left(y_{0}, v_{0}\right)$ и ее зеркального образа $R_{0}\left(y_{0}, v_{0}\right)=$ $\left(1-y_{0},-v_{0}\right) \in \mathscr{G}^{+}$относительно поршня в интервале $(0, \tau)$. Ввиду $(2.13)$ и $(2.26)$ эти две точки будут сталкиваться со стенками и поршнем в моменты времени, отличаюшиеся самое большее на $O\left(\varepsilon_{0}\right)$. И их скорости будут также отличаться самое большее на $O\left(\varepsilon_{0}\right)$; следовательно, в момент времени $T$ они будут располагаться почти симметрично относительно поршня, с точностью $O\left(\varepsilon_{0}\right)$. Из этого вытекает $(2.14)$.

Отметим, что за данное время $T$ две упомянутые вьше движушиеся точки могли испытать разное число столкновений, поскольку одна точка может столкнуться с поршнем или со стенкой, в то время как другая точка может только быть близка к столкновению. Для того чтобы позаботиться о такой возможности, мы ввели в (P5') специальное расстояние $d_{Y}$. Предложение 2.10 доказано.

В следуюшей теореме мы объединяем наши главные результаты.

ТеОРема 2.12. Пусть $T>0$ задано. Если начальная плотность $\pi_{0}(y, v)$ удовлетворяет условиям (P1)-(P5) с достаточно мальим $\varepsilon_{0}$, то

(а) решение наших гидродинамических уравнений (H1)-(H4) существует и единственно на интервале $(0, T)$;

(b) плотность $\pi(y, v, \tau)$ удовлетворяет $\left(\mathrm{P} 1^{\prime}\right)-\left(\mathrm{P} 5^{\prime}\right)$ при всех $0<\tau<T$;

(c) скорость поршня и его ускорение остаются мальмми, $|W(\tau)|=O\left(\varepsilon_{0}\right)$ u $|A(\tau)|=O\left(\varepsilon_{0}\right) ;$ положсение пориня остается близким к средней точке 0.5 в том смысле, что $|Y(\tau)-0.5|=O\left(\varepsilon_{0}\right)$ при всех $0<\tau<T$;

(d) $\left|\tau_{k}-k / v_{\max }\right|=O\left(\varepsilon_{0}\right)$ nри всеx $1 \leqslant k \leqslant T v_{\max }$, u ecлu $T v_{\max }>2$, то и $\left|\tau_{*}-\tau_{2}\right|=O\left(\varepsilon_{0}\right)$. 
СлЕДСТВИЕ 2.13. Если $\varepsilon_{0}=0$, так что начальная плотность $\pi_{0}(y, v)$ симметрична относительно поршня, то решение тривиально: $Y(\tau) \equiv 0.5$ и $W(\tau) \equiv 0$ при всех $\tau>0$.

Наконец, объясним причину, по которой мы предполагаем, что все кривые разрыва начальной плотности $\pi(y, v)$ должны иметь положительный наклон. Казалось бы, можно позволить $\pi(y, v)$ иметь более обшие линии разрьва, например, разрешить такой плотности быть гладкой для $v_{\min }<|v|<v_{\max }$ и резко падать в 0 при $v=v_{\min }$ и $v=v_{\max }$. Следуюший пример показьвает, почему это неприемлемо.

ПРИмЕР. Предположим, что начальная плотность $\pi_{0}(y, v)$ имеет горизонтальную прямую разрьва $v=v_{0}\left(\right.$ скажем, $v_{0}=v_{\min }$ или $\left.v_{0}=v_{\max }\right)$. После взаимодействия с поршнем образ этой прямой разрьва может осциллировать вверх или вниз из-за флуктуаций ускорения поршня (рис. 3). Со временем эта осциллирующая кривая будет “путешествовать” к стенке и возврашаться назад к поршню, испытывая на своем пути некоторые искажения из-за разности скоростей в ее точках (рис. 3). Когда эта кривая возврашается к поршню, у нее могут появиться “точки поворота”, где касательная к ней вертикальна, или даже вертикальные отрезки положительной длины. Так появляются нежелательные сингулярности или даже разрьвы скорости и ускорения поршня. То же явление может также произойти, когда линия разрыва начальной плотности $\pi_{0}(y, v, \tau)$ имеет отрицательньй наклон.

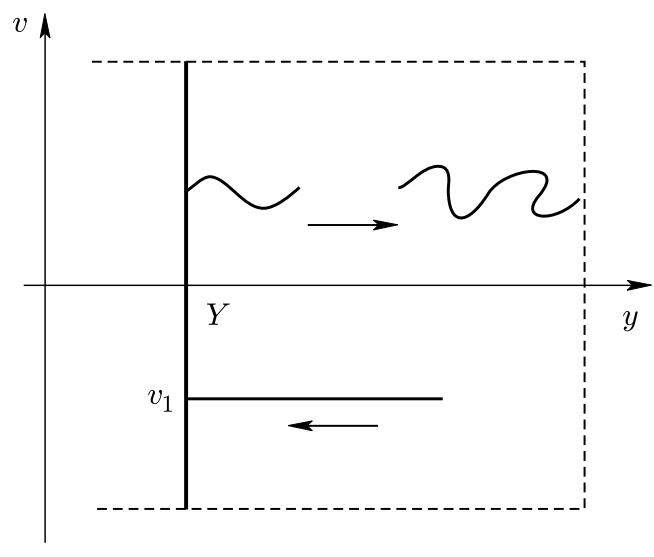

Рис. 3. Горизонтальная прямая разрьва (внизу) уходит от поршня осциллирующей кривой (вверху)

\section{§ 3. Динамика перед первым перестолкновением}

В этом параграфе мы начинаем изучение механической модели поршня в идеальном газе, описанной в $\S 1$. Мы покажем, что случайная траектория поршня, задаваемая функциями $Y_{L}(\tau, \omega)=X_{L}(\tau L, \omega) / L$ и $W_{L}(\tau, \omega)=V_{L}(\tau L / \omega)$, см. (1.6), сходится на интервале без столкновений $\left(0, \tau_{1}\right)$ при $L \rightarrow \infty$ по вероятности к решению $Y(\tau)$ и $W(\tau)$ гидродинамических уравнений, найденных в предыдущем параграфе. 
СоГлАшЕнИЕ. Для краткости обозначений мы не будем указьвать на зависимость от $L$ и $\omega$ в наших выражениях, если это не приведет к путанице. Например, мы будем писать $X(t)$ и $V(t)$ вместо $X_{L}(t, \omega)$ и $V_{L}(t, \omega)$ соответственно.

Мы будем работать с микроскопическим временем $t$. Прежде всего мы определим “микроскопическую” плотность газа, которую мы будем обозначать $p(x, v, t)$ для всех $t \geqslant 0$. Для $t=0$ мы полагаем $p(x, v, 0)=\pi_{0}(x / L, v)$, см. (1.7). Для $t>0$ эволюция плотности определяется правилами, подобньми (H1)-(Н3): свободное движение между столкновениями,

$$
p(x, v, t)=p(x-v s, v, t-s)
$$

для $s>0$ такого, что $x-v r \notin\{0, X(t-r), L\}$ для всех $r \in(0, s)$; отражения от стенок,

$$
p(0, v, t)=p(0,-v, t) \quad \text { и } p(L, v, t)=p(L,-v, t)
$$

и упругие столкновения с поршнем,

$$
p(X(t) \pm 0, v, t)=p(X(t) \pm 0,2 V(t)-v, t) .
$$

Так как последнее равенство включает в себя случайные функции $X(t)$ и $V(t)$, плотность $p(x, v, t)$ будет зависеть от $\omega$, т.е. она будет случайной функцией.

Эволющия плотности $p(x, v, t)$ может быть описана с помощью однопараметрического семейства преобразований $F^{t}$, подобного семейству $\mathscr{F}^{\tau}$, определенному в $§ 2$. Пусть $(x, v)$ - точка области

$$
G:=\{(x, v): 0 \leqslant x \leqslant L\} .
$$

Ее траектория $\left(x_{t}, v_{t}\right)$ для $t>0$ определяется как свободное движение внутри сосуда: $\dot{x}_{t}=v_{t}$ и $\dot{v}_{t}=0$ при $x_{t} \notin\{0, X(t), L\}$, отражениями от стенок: $\left(x_{t+0}, v_{t+0}\right)=$ $\left(x_{t-0}, v_{t-0}\right)$ при $x_{t-0} \in\{0, L\}$, и столкновениями с поршнем:

$$
\left(x_{t+0}, v_{t+0}\right)=\left(x_{t-0}, 2 V(t)-v_{t-0}\right)
$$

при $x_{t-0}=X(t)$. Семейство преобразований $F^{t}$ определяется как $F^{t}\left(x_{0}, v_{0}\right)=\left(x_{t}, v_{t}\right)$ для $t>0$. Мы будем также использовать запись $F^{-t}\left(x_{t}, v_{t}\right)=\left(x_{0}, v_{0}\right)$. Теперь мы просто получаем:

$$
p\left(x_{t}, v_{t}, t\right)=p\left(F^{-t}\left(x_{t}, v_{t}\right), 0\right)=p\left(x_{0}, v_{0}, 0\right) .
$$

Заметим, что для каждого $t>0$ отображение $F^{t}$ есть биекция на $G$ и оно сохраняет площадь, т.е.

$$
\operatorname{det}\left|D F^{t}(x, v)\right|=1 \text {. }
$$

Подчеркнем, что преобразование $F^{t}$, точно так же как и плотность $p(x, v, t)$, случайно, т.е. зависит от $\omega$. 
ЗАмечАнИЕ. Скорость поршня $V(t)$ является кусочно постоянной функцией, задаваемой в моменты столкновения с атомами газа по правилам (1.2)-(1.3). Если $t-$ момент такого столкновения, тогда $V(t)$ в уравнении (3.3) должно быть заменено средним односторонних предельных значений $(V(t+0)+V(t-0)) / 2$. Эта модификация важна, так как она делает правило (3.3) эквивалентным $(1.2)-(1.3)$, когда $\left(x_{t}, v_{t}\right)$ представляет реальную частицу газа массы $m$. В противном случае это соответствует движению частицы нулевой массы, и мы можем называть такую частицу виртуальной.

В силу $(\mathrm{P} 4)$ начальная плотность $p(x, v, 0)$ может быть положительной только в области

$$
G^{+}:=\left\{(x, v): 0 \leqslant x \leqslant L, v_{\min } \leqslant|v| \leqslant v_{\max }\right\},
$$

которая содержит все частищы газа при $t=0$. Для любого $t>0$ область $G^{+}(t):=$ $F^{t}\left(G^{+}\right)$содержит все реальные частицы газа в момент времени $t$ и $p(x, v, t)=0$ для всех $(x, v) \notin G^{+}(t)$.

Для каждой точки $(x, v) \in G$ и $t>0$ мы определяем число столкновений с поршнем в интервале $(0, t)$

$$
N(x, v, t)=\#\left\{s \in(0, t): x_{s}=X(s), v_{s} \neq V(s)\right\} .
$$

Затем мы разбиваем область $G$ на подобласти

$$
G_{n}(t):=\left\{F^{t}(x, v):(x, v) \in G \& N(x, v, t)=n\right\}
$$

и полагаем $G_{n}^{+}(t):=G^{+}(t) \cap G_{n}(t)$. Область $G_{n}^{+}(t)$ занята точками, испытавшими в точности $n$ столкновений в интервале времени $(0, t)$.

Мы подчеркиваем, что наши преобразования $F^{t}$ и области $G_{n}(t)$ и $G_{n}^{+}(t)$ зависят от $\omega$, т.е. случайны. Заметим, однако, что они полностью определяются траекторией поршня, т.е. функщией $X(s), 0<s<t$.

Семейство преобразований $\mathscr{F}^{\tau}: \mathscr{G} \rightarrow \mathscr{G}$, введенное в $\S 2$, индуцирует детерминистское семейство $\widetilde{F}^{t}: G \rightarrow G$, определяемое следующим образом. Если $\mathscr{F}^{\tau}(y, v)=$ $\left(y_{\tau}, v_{\tau}\right)$, то $\widetilde{F}^{\tau L}(y L, v):=\left(y_{\tau} L, v\right)$. Преобразование $\widetilde{F}^{t}$ определяет детерминистскую функцию плотности на $G$ как $\widetilde{p}(x, v, t):=p\left(\widetilde{F}^{t}(x, v), 0\right) ;$ с плотностью $\pi(y, v, \tau)$ из 2 ее связывает соотношение $\widetilde{p}(x, v, t)=\pi(x / L, v, t / L)$. Мы также положим $\widetilde{G}^{+}(t):=$ $\mathscr{F}^{t}\left(G^{+}\right)$.

Частицы газа в $G_{0}(t)$ определяют пуассоновский процесс, как показьвает следующая лемма. Пусть $\omega \in \Omega$ и $t>0$. Фиксируем траекторию поршня $X(s), 0<s<t$. Она полностью определяет область $G_{0}(t)$ и плотность $p(x, v, t)$.

ЛЕмма 3.1. Условное распределение частии газа в $G_{0}(t)$ (при заданной траектории поршня $X(s), 0<s<t)$ является пуассоновским с плотностью $L^{2} p(x, v, t)$.

ДокАЗАТЕльство. Пусть $D \subset G_{0}(t)$ - произвольная область. Тогда прообраз $F^{-(t-s)}(D)$ находится на положительном расстоянии от $X(s)$ при всех $s \in(0, t)$. Следовательно, частищы, выходящие из области $F^{-1}(D)$ и заканчивающие свое движение в $D$, не могут воздействовать на поршень в течение интервала времени $(0, t)$. Таким образом, число частиц в $D$ в момент времени $t$, будучи равным числу частиц в $F^{-1}(D)$ 
в момент времени 0, не зависит от траектории поршня и, значит, является пуассоновской случайной величиной с параметром

$$
\lambda_{D}(t)=L^{2} \iint_{F^{-1}(D)} p(x, v, 0) d x d v=L^{2} \iint_{D} p(x, v, t) d x d v
$$

Равенство этих интегралов следует из (3.5) и (3.6).

ЗАмЕчАНИЕ. Для любой области $D \subset G_{0}(t)$ ее прообраз $F^{-1}(D)$ действительно не зависит от $\omega$. В самом деле, пусть $F_{0}^{t}$ - другое семейство преобразований на $G$, определяемое свободным движением на всем интервале $0<x<L$ и упругими отражениями только на стенках $x=0$ и $x=L$ (как будто поршня нет). Тогда мы имеем $F^{-1}(D)=F_{0}^{-1}(D)$ для любой области $D \subset G_{0}(t)$.

Для $n \geqslant 1$ мы определяем $T_{n}$ как наименшшее время, через которое поршень взаимодействует с точками из $G_{n}^{+}(t)$ (таким образом, возникает область $G_{n+1}^{+}(t)$ ), или, эквивалентно,

$$
T_{n}=\sup _{t>0}\left\{G_{n+1}^{+}(t)=\varnothing\right\} .
$$

Временные моменты $T_{n}=T_{n}(\omega)$ являются случайными аналогами моментов $\tau_{n}$ из $\S 2$. $\mathrm{B}$ частности, $T_{1}$ есть время первого перестолкновения в системе (с реальной или виртуальной частищей).

ЛЕмма 3.2. Для всех $\omega \in \Omega$

$$
T_{1} \leqslant T_{1, \max }:=L / v_{\max }
$$

ДокАЗАТЕльство. Самые быстрые частицы $(x, v) \in G^{+}$, которые сталкиваются с поршнем в момент времени 0 , будут двигаться со скоростью $v_{\max }$ и снова столкнутся с поршнем в момент $t$, для которого $v_{\max } t+|X(t)-L / 2|=L$. Для всех таких $t$ мы имеем

$$
T_{1} \leqslant t=\frac{L-|X(t)-L / 2|}{v_{\max }} .
$$

Лемма доказана.

В течение интервала времени $\left(0, T_{1}\right)$ поршень взаимодействует с частицами в $G_{0}^{+}(t)$. Обозначим

$$
\begin{array}{r}
\mathscr{X}_{0}(t)=\left\{(x, v): x=X(t)+0,-v_{\max }<v<-v_{\min }\right\} \\
\cup\left\{(x, v): x=X(t)-0, v_{\min }<v<v_{\max }\right\}
\end{array}
$$

объединение двух односторонних окрестностей поршня, содержаших все "входяшие" частицы, которые вот-вот столкнутся с поршнем.

Для того чтобы изучить динамику поршня в интервале без перестолкновений, удобно предположить, что поршень движется достаточно медленно и взаимодействует только с начальными частицами, которые начинают движение в $G^{+}$при $t=0$. Подынтервал $\left(0, S_{1}\right) \subset\left(0, T_{1}\right)$, где поршень удовлетворяет этим требованиям, будет называться "медленным" интервалом. 
ОПРЕДЕЛЕНИЕ. Мы определим $\left(0, S_{1}\right) \subset\left(0, T_{1}\right)$ как максимальный временной интервал, в котором

(a) $|V(t)|<v_{\min }$;

(b) $\mathscr{X}_{0}(t) \subset G_{0}(t)$.

Заметим, что по условию (b) все входяшие частицы, близкие к соударению с поршнем в момент времени $t$, начали движение в $G^{+}$в нулевой момент времени и вообше не взаимодействовали с поршнем во временном интервале $(0, t)$.

Почти все наши рассмотрения в этом параграфе будут ограничены во времени "медленным" интервалом $\left(0, S_{1}\right)$. Но в конце параграфа мы увидим, что для типичных $\omega$ "медленньй" интервал совпадает со всем интервалом $\left(0, T_{1}\right)$, т.е. $S_{1}=T_{1}$.

Для каждого $t \in\left(0, S_{1}\right)$ мы определяем плотность столкновений частиц на поршне $q(v, t) \mathrm{KaK}$

$$
q(v, t)= \begin{cases}p(X(t)-0, v, t) & \text { при } v>0, \\ p(X(t)+0, v, t) & \text { при } v<0\end{cases}
$$

(ср. с (2.17)). Затем мы определяем, подобно (2.10)-(2.21) в $§ 2$,

$$
\begin{aligned}
Q_{0}(t) & =\int \operatorname{sign} v \cdot q(v, t) d v, \\
Q_{1}(t) & =\int v \operatorname{sign} v \cdot q(v, t) d v, \\
Q_{2}(t) & =\int v^{2} \operatorname{sign} v \cdot q(v, t) d v .
\end{aligned}
$$

Так как $p(x, v, t)$, ограниченная на область $G_{0}(t)$, совпадает с $\widetilde{p}(x, v, t)=\pi(x / L, v$, $t / L)$, из условий $\left(\mathrm{P} 1^{\prime}\right)-\left(\mathrm{P} 2^{\prime}\right)$ вытекает следуюшая лемма.

ЛЕмма 3.3. Плотность $p(x, v, t)$, ограниченная на область $G_{0}(t)$, является кусочно $C^{1}$-гладкой функиией на $(x, v)$-плоскости. Кроме того, $|\partial p(x, v, t) / \partial x| \leqslant$ $D_{1}^{\prime} / L$. Линии разрыва функиии $p(x, v, t)$ внутри области $G_{0}(t)$ имеют наклон порядка $O(1 / L)$ (для больших $L$ они почти параллельны оси $x)$.

В силу этой леммы величины $Q_{i}, i=0,1,2$, как функции положения поршня $X$, являются гладкими и имеют производные

$$
\left|\frac{\partial Q_{i}}{\partial X}\right| \leqslant \frac{\text { const }}{L}
$$

где const является глобальной постоянной.

Следующая теорема дает основную техническую оценку этого параграф̆а.

ТеОрема 3.4. Для достаточно больших L существует множсество $\Omega_{0}^{*} \subset \Omega$ начальных конфигурачий частич газа такое, что

(i) для некоторой постоянной $c>0$

$$
\mathrm{P}\left(\Omega_{0}^{*}\right)>1-L^{-c \ln \ln L}
$$


(ii) для каждой конфигурации $\omega \in \Omega_{0}^{*}$ и каждого интервала времени

$$
(t, t+\Delta t) \subset\left(0, S_{1}\right)
$$

такого, что

$$
\frac{1}{L^{2}}<\Delta t<\frac{1}{L^{2 / 3} \ln L}
$$

изменение скорости пориня в $(t, t+\Delta)$ удовлетворяет равенству

$$
V(t+\Delta)-V(t)=\mathscr{D}(t) \Delta t+\chi
$$

$2 \partial e$

$$
\mathscr{D}(t) / a=Q_{0}(t) V^{2}(t)-2 Q_{1}(t) V(t)+Q_{2}(t)
$$

$u$

$$
|\chi| \leqslant C \frac{\ln L \sqrt{\Delta t}}{L}
$$

с некоторой постоянной $C>0$.

ЗАмЕчАниЕ. Функция $\mathscr{D}(t) / a$ в $(3.16)$ - это случайный аналог квадратичного полинома из (2.18). Член $\mathscr{D}(t) \Delta t$ в (3.15) есть главная (“детерминистская") компонента динамики скорости поршня. Член $\chi$ представляет случайные флуктуации.

ДокАЗАТЕЛЬСТво. Множество $\Omega_{0}^{*}$ будет состоять из всех конфигураций, удовлетворяюших некоторым требованиям. Мы начнем с предварительных требований.

Рассмотрим дискретное множество моментов времени $t_{i}=i / L^{2}$, где $i=0,1, \ldots, I$ и $I=\left[T_{1, \max } L^{2}\right]$. Разобьем область $G$ на полосы $S_{j}:=\left\{(x, v): j / L^{2} \leqslant x<(j+1) / L^{2}\right\}$, где $j=0,1, \ldots, L^{3}-1$. Для каждых $i$ и $j$ обозначим через $N_{i, j}$ число частиц газа в области $S_{j} \cap G_{0}\left(t_{i}\right)$ в момент времени $t_{i}$. Наши предварительные требования состоят в том, что

$$
N_{i, j} \leqslant \ln L
$$

для всех $0 \leqslant i \leqslant I$ и $0 \leqslant j<L^{3}$. Мы видим, что $N_{i, j}$ равняется числу частиц газа в области $F^{-t_{i}}\left(S_{j} \cap G_{0}\left(t_{i}\right)\right)$ в нулевой момент времени и

$$
F^{-t_{i}}\left(S_{j} \cap G_{0}\left(t_{i}\right)\right) \subset F_{0}^{-t_{i}}\left(S_{j}\right) .
$$

Значит, $N_{i, j}$ не превосходит числа частиц газа в $F_{0}^{-t_{i}}\left(S_{j}\right)$ в момент времени 0, которьй мы обозначим $\widetilde{N}_{i, j}$. Далее, $\widetilde{N}_{i, j}$ - пуассоновская случайная величина, параметр $\lambda_{i, j}$ которой ограничен:

$$
\lambda_{i, j} \leqslant L^{2} \pi_{\max }\left|S_{j}\right| \leqslant 2 \pi_{\max }\left(v_{\max }-v_{\min }\right) .
$$

Согласно следствию А.4 для каждых $i, j$ наше требование (3.18) не будет вьполнено с вероятностью $<L^{-d \ln \ln L}$ для некоторого $d>0$. Обшее число пар $i, j$ равняется $L^{3} I=L^{5} T_{1, \max } \leqslant L^{6} /\left(2 v_{\max }\right)$. Следовательно, все наши предварительные требования выполнены с вероятностью $>1-L^{-c^{\prime}} \ln \ln L$ длянекоторой глобальной постоянной $c^{\prime}>0$. 
Теперь мы возврашаемся к доказательству (3.14)-(3.16). Пусть $i=\left[L^{2} t\right]$ и $t_{1}=(i+1) / 2$. Заметим, что $t_{1}-t \leqslant L^{-2}$. Из наших предварительных требований можно легко вьвести, что число частиц газа, сталкиваюшихся с поршнем в интервале времени $\left(t, t_{1}\right)$, меньше const $\cdot \ln L$. Следовательно, скорость поршня $V$ не меняется больше чем на const $\cdot \ln L \cdot L^{-2}$ в течение этого интервала. Эта величина меньше гранищы на $\chi$ в (3.17) для всех $\Delta t$, удовлетворяюших (3.14). Поэтому мы можем игнорировать интервал $\left(t, t_{1}\right)$ и предположить, что $t=t_{1}$. Отметим, что величины $Q_{0}, Q_{1}, Q_{2}$ в (3.16) будут изменяться также в результате подстановки $t=t_{1}$, но только на величину $<$ const $/ L^{3}$ в силу леммы 3.3 . Очевидно, что изменение тоже может быть проигнорировано. Поэтому мы предположим, что $t=i / L^{2}$ для некоторого $i=0,1, \ldots, I$.

Теперь мы сформулируем наши главные требования. Мы снова разобьем ось $x$ на интервалы $S_{j}:=\left\{j / L^{2} \leqslant x<(j+1) / L^{2}\right\}$, где $j=0,1, \ldots, L^{3}-1$. Для каждого $j$ полагаем $x_{j}=j / L^{2}$. Для каждого целого $p,|p| \leqslant v_{\min } L^{2}$, полагаем $v_{p}=p / L^{2}$ и для каждого целого $1 \leqslant q \leqslant L^{2}$ полагаем $d_{q}=q / L^{2}$. Теперь для каждой тройки $(j, p, q)$ мы определяем две трапецеидальные области на $(x, v)$-плоскости (см. рис. 4 ниже):

$$
\begin{aligned}
& D_{j, p, q}^{-}=\left\{(x, v): \frac{v-v_{p}}{x-x_{j}}<-\frac{1}{d_{q}}, v_{\min }<v<v_{\max }\right\}, \\
& D_{j, p, q}^{+}=\left\{(x, v): \frac{v-v_{p}}{x-x_{j}}<-\frac{1}{d_{q}},-v_{\max }<v<-v_{\min }\right\}
\end{aligned}
$$

(здесь $-1 / d_{q}-$ наклон скошенных сторон этих трапеций). Для каждой тройки $(j, p, q)$ мы определяем две полосы:

$$
\begin{aligned}
& U_{j, p, q}^{-}=\left\{(x, v):\left|x-x_{j}+d_{q}\left(v-v_{p}\right)\right|<10 v_{\max } / L^{2}, v_{\min }<v<v_{\max }\right\}, \\
& U_{j, p, q}^{+}=\left\{(x, v):\left|x-x_{j}+d_{q}\left(v-v_{p}\right)\right|<10 v_{\max } / L^{2},-v_{\max }<v<-v_{\min }\right\} .
\end{aligned}
$$

Заметим, что $U_{j, p, q}^{ \pm}$являются окрестностями наклонных сторон трапеший $D_{j, p, q}^{ \pm}$.

Рассмотрим все моменты времени $t_{i}=i / L^{2}$ для $i=0,1, \ldots, I$. Обозначим через $N_{i, j, p, q}^{ \pm}$число частиц в области $F_{0}^{-t_{i}}\left(D_{j, p, q}^{ \pm}\right)$в момент времени 0 и через $M_{i, j, p, q}^{ \pm}$- число частищ в области $F_{0}^{-t_{i}}\left(U_{j, p, q}^{ \pm}\right)$в момент времени 0 . Они являются пуассоновскими случайными величинами. Параметры величин $N_{j, p, q}^{ \pm}$суть

$$
\lambda_{i, j, p, q}^{ \pm}=\mathrm{E}\left(N_{i, j, p, q}^{ \pm}\right)=L^{2} \int_{F_{0}^{-t_{i}}\left(D_{j, p, q}^{ \pm}\right)} p(x, v, 0) d x d v
$$

Можно непосредственно проверить, что $\lambda_{i, j, p, q}^{ \pm}$равномерно ограничен низу положительной постоянной (даже для наименьшего $d_{p}$, т.е. для $d_{p}=1 / L^{2}$ ) в силу предположения (1.8) на начальную плотность. Заметим также, что параметры для $M_{i, j, p, q}^{ \pm}$ равномерно ограничены сверху величиною

$$
\mathrm{E}\left(M_{i, j, p, q}^{ \pm}\right)<\pi_{\max }\left|U_{i, j, p, q}^{ \pm}\right|<20 v_{\max }\left(v_{\max }-v_{\min }\right) \pi_{\max } .
$$


Наши главные требования состоят в том, что

$$
\left|N_{i, j, p, q}^{ \pm}-\lambda_{i, j, p, q}^{ \pm}\right| \leqslant \ln L \sqrt{\lambda_{i, j, p, q}^{ \pm}}
$$

и

$$
M_{i, j, p, q}^{ \pm} \leqslant \ln L .
$$

По лемме А. 3 и следствию А.4 вероятность того, что хотя бы одно из этих требований не выполняется, будет меньше, чем $L^{-d \ln \ln L}$ с некоторой постоянной $d>0$. Общее число четверок $(i, j, p, q)$ не превьшает $L^{9} T_{1, \max } \leqslant L^{A^{\prime \prime}}$ с некоторьм фиксированньм $A^{\prime \prime}>0$. Следовательно, все наши главные требования вьполняются с вероятностью $>1-L^{-c^{\prime \prime} \ln \ln L}$ для некоторой постоянной $c^{\prime \prime}>0$.

Дополнительно, пусть

$$
Z_{i, j, p, q}^{ \pm}=\sum_{(x, v) \in F_{0}^{-t_{i}}\left(D_{j, p, q}^{ \pm}\right)} v
$$

в момент времени 0. Это есть "интегрированная" пуассоновская случайная величина, как определено в приложении. (Технически, мы требуем здесь, что одна из сторон области должна быть на оси $x$, но может случиться так, что область $F_{0}^{-t_{i}}\left(D_{j, p, q}^{ \pm}\right)$ пересекает либо стенку $x=0$, либо стенку $x=L$, тогда две стороны области лежат на оси $x$; в этом случае нам нужно заменить $t_{i}$ близким временем $t_{i^{\prime}}<t_{i}$ так, чтобы одна сторона области $F_{0}^{-t_{i}}\left(D_{j, p, q}^{ \pm}\right)$лежала целиком на оси $x$, и определить $Z_{i, j, p, q}^{ \pm}$ для момента времени $t_{i}-t_{i^{\prime}}$ вместо 0 ; необходимо произвести некоторые очевидные изменения, но мы опускаем детали.) Равенства, полученные в приложении, дают

$$
\begin{aligned}
\mathrm{E}\left(Z_{i, j, p, q}^{ \pm}\right) & =L^{2} \int_{F_{0}^{-t_{i}}\left(D_{j, p, q}^{ \pm}\right)} v p(x, v, 0) d x d v \\
\mathrm{D}\left(Z_{i, j, p, q}^{ \pm}\right) & =L^{2} \int_{F_{0}^{-t_{i}}\left(D_{j, p, q}^{ \pm}\right)} v^{2} p(x, v, 0) d x d v .
\end{aligned}
$$

Наше последнее требование состоит в том, что

$$
\left|Z_{i, j, p, q}^{ \pm}-\mathrm{E}\left(Z_{i, j, p, q}^{ \pm}\right)\right| \leqslant \ln L \sqrt{\mathrm{D}\left(Z_{i, j, p, q}^{ \pm}\right)}
$$

для всех $i, j, p, q$. Вероятность невыполнения этих требований оценивается точно так же, как и выше, с помошью леммы А.6.

Теперь мы переходим к оценке скорости поршня в интервале времени $(t, t+\Delta t)$. Напомним, что $t=t_{i}$ для некоторого $i=0,1, \ldots, I$.

Схема разложения скорости. Здесь мы получаем общую формулу для скорости поршня, которую используем в доказательствах нескольких теорем. Из законов упругого столкновения следует [19], что

$$
V(t+\Delta t)=(1-\varepsilon)^{k} V(t)+\varepsilon \sum_{j=1}^{k}(1-\varepsilon)^{k-j} v_{j} .
$$


3 десь $k$ - число частиц, сталкивающихся с поршнем в интервале времени $(t, t+\Delta t)$, а $v_{j}-$ их скорости, занумерованные в порядке их столкновений. Уравнение (3.26) может быть легко проверено индукцией по $k$.

Мы модифицируем формулу (3.26) следуюшим образом:

$$
V(t+\Delta t)=(1-\varepsilon k) V(t)+\varepsilon \sum_{j=1}^{k} v_{j}+\chi^{(1)}+\chi^{(2)},
$$

где

$$
\begin{aligned}
& \chi^{(1)}=V(t)\left[(1-\varepsilon)^{k}-1+\varepsilon k\right], \\
& \chi^{(2)}=\varepsilon \sum_{j=1}^{k} v_{j}\left[(1-\varepsilon)^{k-j}-1\right] .
\end{aligned}
$$

Предположим, что флуктуации скорости $V(s)$ на интервале $(t, t+\Delta t)$ ограничены:

$$
\sup _{s \in(t, t+\Delta t)}|V(s)-V(t)| \leqslant \delta V .
$$

Рассмотрим две области на $(x, v)$-плоскости:

$$
\begin{aligned}
& D_{1}=\left\{(x, v): \frac{v-V(t)-(\operatorname{sign} v) \delta V}{x-X(t)}<-\frac{1}{\Delta t}, v_{\min }<|v|<v_{\max }\right\}, \\
& D_{2}=\left\{(x, v): \frac{v-V(t)+(\operatorname{sign} v) \delta V}{x-X(t)}<-\frac{1}{\Delta t}, v_{\min }<|v|<v_{\max }\right\} .
\end{aligned}
$$

Каждая из них является объединением двух трапеций $D_{i}=D_{i}^{+} \cup D_{i}^{-}, i=1,2$, где $D_{i}^{-}$обозначает верхнюю, а $D_{i}^{+}$- нижнюю трапецию, см. рис. 4 .

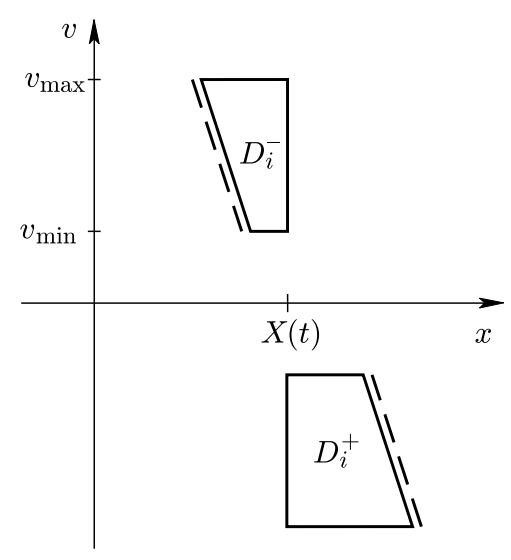

Рис. 4. Область $D_{1}$ ограничена сплошными прямыми. Область $D_{2}$ ограничена пунктирными прямыми 
Заметим, что $D_{1} \subset D_{2}$. Из (3.28) вытекает, что все частищы в области $D_{1}$ обязательно сталкиваются с поршнем в интервале времени $(t, t+\Delta t)$. Более того, траектория каждой точки $(x, v) \in D_{1}$ ударяет поршень за время, меньшее $\Delta t$; следовательно, $D_{1} \subset G_{0}(t)$ по условию (b) в определении "медленного" интервала $\left(0, S_{1}\right)$. Из (3.28) следует также, что все частищы, действительно сталкивающиеся с поршнем в интервале $(t, t+\Delta t)$, содержатся в $D_{2}$ (хотя не обязательно $D_{2} \subset G_{0}(t)$ ).

Теперь мы получим верхнюю границу для $k$. Так как скорости частиц газа и поршня ограничены величиной $v_{\max }$, то все частищы, сталкиваюшиеся с поршнем в интервале $(t, t+\Delta t)$, содержатся в области $S^{\prime} \cap G_{0}(t)$, где

$$
S^{\prime}=\left\{(x, v):|x-X(t)|<2 v_{\max } \Delta t\right\} .
$$

Следовательно, $k$ не превосходит числа частиц в области $F_{0}^{-t}\left(S^{\prime}\right)$ в нулевой момент времени. Из наших предварительных требований вытекает, что

$$
k \leqslant 2 v_{\max } \Delta t L^{2} \ln L .
$$

Это позволяет нам ограничить величины $\chi^{(1)}$ и $\chi^{(2)}$ для больших $L$ следуюшим образом:

$$
\left|\chi^{(u)}\right| \leqslant v_{\max } \varepsilon^{2} k^{2} \leqslant 4 a^{2} v_{\max }^{3}(\ln L)^{2}(\Delta t)^{2}
$$

для $u=1,2$. Из (3.27) также следует, что

$$
\delta V \leqslant 5 a v_{\max } \Delta t \ln L .
$$

Мы обозначим через $k_{r}^{ \pm}$число частиц в областях $D_{r}^{ \pm} \cap G_{0}(t)$ для $r=1,2$ в момент времени $t=t_{i}$. Мы обозначаем также через $k^{-}$число частиц, сталкиваюшихся с поршнем "слева", и через $k^{+}$- такое число "справа" (конечно, $k^{-}+k^{-}=k$ ). Ясно, что

$$
k_{1}^{ \pm} \leqslant k^{ \pm} \leqslant k_{2}^{ \pm}
$$

Так как $D_{1} \subset G_{0}(t)$, то $k_{1}^{ \pm}$равно числу частиц в области $F_{0}^{-t}\left(D_{1}^{ \pm}\right)$в момент времени 0 . Аналогично, $k_{2}^{ \pm}$равно числу частиц в области $F_{0}^{-t}\left(D_{2}^{ \pm} \cap G_{0}(t)\right)$ в момент времени 0.

Трапеции $D_{r}^{ \pm}, r=1,2$, могут быть хорошо апроксимированы некоторьми трапешиями $D_{j, p, q}^{ \pm}$, определенньми ранее в наших основных требованиях. На самом деле, горизонтальные стороны $v= \pm v_{\min }$ и $v= \pm v_{\max }$ являются обшими для всех трапеций, только вертикальная сторона $x=X(t)$ и наклонная сторона нуждаются в аппроксимации. Симметрическая разность между $D_{r}^{ \pm}$и аппроксимирующей $D_{j, p, q}^{ \pm}$будет лежать внутри полос $S_{j}$ и $U_{j, p, q}^{ \pm}$, также определенных ранее. Тогда наши главные требования будут гарантировать, что

$$
k_{1}^{ \pm} \leqslant \lambda_{1}^{ \pm}-\ln L \sqrt{\lambda_{1}^{ \pm}} \text {и } \quad k_{2}^{ \pm} \leqslant \lambda_{2}^{ \pm}+\ln L \sqrt{\lambda_{2}^{ \pm}},
$$

где

$$
\begin{aligned}
& \lambda_{1}^{ \pm}=L^{2} \int_{F_{0}^{-t}\left(D_{1}^{ \pm}\right)} p(x, v, 0) d x d v=L^{2} \int_{D_{1}^{ \pm}} p(x, v, t) d x d v \\
& \lambda_{2}^{ \pm}=L^{2} \int_{F_{0}^{-t}\left(D_{2}^{ \pm} \cap G_{0}(t)\right)} p(x, v, 0) d x d v \leqslant \lambda_{1}^{ \pm}+L^{2} \pi_{\max }\left|D_{2}^{ \pm} \backslash D_{1}^{ \pm}\right|
\end{aligned}
$$


(мы воспользовались ограниченностью плотности (1.9)). Площадь области $D_{2}^{ \pm} \backslash D_{1}^{ \pm}$ ограничена:

$$
\left|D_{2}^{ \pm} \backslash D_{1}^{ \pm}\right|<4 v_{\max } \delta V \Delta t
$$

Из (1.9) также следует, что

$$
\lambda_{1}^{ \pm} \leqslant L^{2} \pi_{\max }\left|D_{1}^{ \pm}\right| \leqslant 2 \pi_{\max } v_{\max }^{2} L^{2} \Delta t .
$$

Комбинируя полученные оценки, приходим к неравенству

$$
\left|k^{ \pm}-\lambda_{1}^{ \pm}\right| \leqslant \text {const } \cdot\left(L \ln L \sqrt{\Delta t}+L^{2} \delta V \Delta t\right) .
$$

Теперь мы обратимся к величине

$$
Z=\sum_{j=1}^{k} v
$$

также входящей в основное уравнение (3.27). Снова можно воспользоваться разложением $Z=Z^{-}+Z^{+}$, где $Z^{-}$и $Z^{+}$обозначают суммы скоростей частищ, сталкиваюшихся с поршнем "слева" и "справа" соответственно. Мы полагаем

$$
Z_{1}^{ \pm}=\sum_{(x, v) \in D_{1}^{ \pm}} v
$$

(в момент времени $t$ ). Анализ, подобньй предыдущему, и требование (3.25) вместе с формулами (3.23) и (3.24) дают

$$
\left|Z^{ \pm}-\mathrm{E}\left(Z_{1}^{ \pm}\right)\right| \leqslant \text {const } \cdot\left(L \ln L \sqrt{\Delta t}+L^{2} \delta V \Delta t\right) .
$$

Теперь мы комбинируем (3.27) со всеми имеюшимися оценками и получаем, что

$$
V(t+\Delta)-V(t)=-\varepsilon \lambda_{1} V(t)+\varepsilon \mathrm{E}\left(Z_{1}\right)+\chi^{(3)},
$$

где

$$
\begin{gathered}
\lambda_{1}=\lambda_{1}^{+}+\lambda_{1}^{-}=L^{2} \int_{D_{1}} p(x, v, t) d x d v \\
\mathrm{E}\left(Z_{1}\right)=\mathrm{E}\left(Z_{1}^{+}\right)+\mathrm{E}\left(Z_{1}^{-}\right)=L^{2} \int_{D_{1}} v p(x, v, t) d x d v, \\
\left|\chi^{(3)}\right| \leqslant \text { const } \cdot\left[L^{-1} \ln L \sqrt{\Delta t}+\delta V \Delta t+(\ln L)^{2}(\Delta t)^{2}\right] .
\end{gathered}
$$

Использование леммы 3.3 (как мы уже это делали при вьводе (3.13) для того, чтобы ограничить возможные флуктуации плотности $p(x, v, t)$ в $\left.D_{1}\right)$ дает

$$
\begin{aligned}
\int_{D_{1}} p(x, v, t) d x d v & =\left(Q_{1}(t)-Q_{0}(t)\right) V(t) \Delta t+\chi^{(4)}, \\
\int_{D_{1}} v p(x, v, t) d x d v & =\left(Q_{2}(t)-Q_{1}(t)\right) V(t) \Delta t+\chi^{(5)}
\end{aligned}
$$


где

$$
\left|\chi^{(u)}\right| \leqslant \text { const } \cdot\left[(\Delta t)^{2} / L+\delta V \Delta t\right]
$$

c $u=4,5$.

Таким образом, мы получим

$$
V(t+\Delta t)-V(t)=\mathscr{D}(t) \Delta t+\chi
$$

где

$$
|\chi| \leqslant \text { const } \cdot\left[L^{-1} \ln L \sqrt{\Delta t}+\delta V \Delta t+(\ln L)^{2}(\Delta t)^{2}\right]
$$

Используя (3.14) и (3.33), легко включить второе и третье слагаемое в (3.42) в первое. Теорема 3.4 доказана.

Для следующей теоремы мы перепишем (Р5) в микроскопических координатах:

$$
|p(x, v, 0)-p(L-x,-v, 0)|<\varepsilon_{0} .
$$

Мы также предполагаем, что начальная скорость поршня $V(0)=W(0)$, а не 0 , см. замечание $\S 2$ и другое замечание ниже.

Теорема 3.5. Предположим, что $\varepsilon_{0}>0$ достаточно мало. Для всех достаточно больших $L$, для каждой конфигурачии $\omega \in \Omega_{0}^{*}$ и для всех $t \in\left(0, S_{1}\right)$

(i) существует такая постоянная $B>0$, что

$$
|V(t)|<B \varepsilon_{0}
$$

(ii) существует такая постоянная $C_{0}>0$, что

$$
\left|V(t)-V_{0}(t)\right|<\frac{C_{0}(\ln L)^{3 / 2}}{L^{2 / 3}},
$$

где $V_{0}(t)$ определяется равенством

$$
V_{0}(t)=\frac{Q_{1}(t)-\sqrt{Q_{1}^{2}(t)-Q_{0}(t) Q_{2}(t)}}{Q_{0}(t)},
$$

когда $Q_{0}(t) \neq 0$, и равенством

$$
V_{0}(t)=\frac{Q_{2}(t)}{2 Q_{1}(t)}
$$

в противном случае. 
ДокАЗАТЕЛЬСТво. Мыначнем с доказательства (i) и получим (ii) как "побочньй результат".

Предположим, что (i) неверно, и пусть $t_{*}<S_{1}$ - время, при котором (3.44) нарушается впервые, т.е.

$$
\left|V\left(t_{*}+0\right)\right| \geqslant B \varepsilon_{0}
$$

Так как (3.44) верно при $t<t_{*}$, положение поршня удовлетворяет неравенству

$$
|X(t)-L / 2| \leqslant B \varepsilon_{0} t
$$

для всех $t<t_{*}$. Предположим на время, что поршень не двигался вовсе, т.е. $X(s)=$ $L / 2$ для всех $0<s<t$. Тогда по (3.43) плотность $q(v, t)$ газа "на поршне" была бы почти симметрической, т.е.

$$
|q(v, t)-q(-v, t)|<\varepsilon_{0} .
$$

Следовательно, мы имели бы

$$
\left|Q_{i}(t)\right|<C_{1} \varepsilon_{0}, \quad i=0,2,
$$

с $C_{1}=v_{\max }^{3}$. Мы подчеркиваем, что $C_{1}$ не зависит от выбора $B$ в (3.44). Далее мы введем некоторые дополнительные постоянные $C_{i}, i \geqslant 2$, такие, что ни одна из них не будет зависеть от $B$.

Если же в действительности поршень двигается и покрьвает расстояние $X(t)-$ $L / 2$, то из (3.13) и (3.50) следует

$$
\left|Q_{i}(t)\right|<C_{1} \varepsilon_{0}+C_{2} L^{-1}|X(t)-L / 2|, \quad i=0,2,
$$

с некоторой постоянной $C_{2}>0$. В то же самое время $Q_{1}(t)$ остается ограниченной сверху и снизу положительными постоянными для всех $t<t_{*}$ :

$$
0<Q_{1, \min } \leqslant Q_{1}(t) \leqslant Q_{1, \max }<\infty,
$$

где $Q_{1, \min }$ и $Q_{1, \max }$ - постоянные, определяемые $\pi_{\min }$ и $\pi_{\max }$ в (P3).

Из (3.49), (3.51) и (3.8) мы получаем

$$
\left|Q_{i}(t)\right|<\left(C_{1}+B v_{\max }^{-1}\right) \varepsilon_{0}, \quad i=0,2,
$$

для всех $t<t_{*}$. Из этого и из (3.52) следует, что квадратичньй полином (3.16) имеет два действительных корня и наименьший из них (более близкий к нулю) дается равенствами (3.46), (3.47), ср. (2.24)-(2.25) из §2. Ввиду (2.22) и (3.52) мы получаем, что

$$
\left|V_{0}(t)\right|<C_{3} \varepsilon_{0}+C_{4} L^{-1}|X(t)-L / 2|
$$

для всех $t<t_{*}$ и для некоторых постоянных $C_{3}, C_{4}>0$ (не зависяших от выбора $B$ ).

Мы замечаем, что из (3.54) и (3.8) следует ограниченность $V_{0}(t)$ :

$$
\left|V_{0}(t)\right| \leqslant \text { const }=C_{3} \varepsilon_{0}+C_{4} v_{\max }^{-1}
$$

для всех $t<t_{*}$. 
Далее, нам необходимо оценить производную $d V_{0}(t) / d t$. (Замечание: функция $V_{0}(t)$, определенная равенствами (3.46), (3.47), непрерьвна и кусочно дифференцируема, ее не следует путать со скоростью поршня $V(t)$, которая кусочно постоянна и, следовательно, даже не непрерьвна.) Из (3.13) следует, что

$$
\left|\frac{d Q_{i}(t)}{d t}\right|=\left|\frac{d Q_{i}(t)}{d X} \cdot V(t)\right| \leqslant \frac{\text { const } \cdot \varepsilon_{0}}{L}
$$

для $i=0,1,2$. Дифференцирование квадратного уравнения

$$
Q_{0}(t) V_{0}^{2}(t)-2 Q_{1}(t) V_{0}(t)+Q_{2}(t)=0
$$

по $t$ дает

$$
\frac{d V_{0}(t)}{d t}=\frac{\left(d Q_{0} / d t\right) V_{0}^{2}-2\left(d Q_{1} / d t\right) V_{0}+\left(d Q_{2} / d t\right)}{2\left(Q_{1}-Q_{0} V_{0}\right)} .
$$

Из (2.22), (3.52), (3.55) и (3.56) мы имеем

$$
\left|\frac{d V_{0}(t)}{d t}\right| \leqslant \frac{E_{0} \varepsilon_{0}}{L}
$$

где $E_{0}>0$ - постоянная.

Теперь рассмотрим определенную в (3.16) величину $\mathscr{D}$ как функщию от $V$ (с фиксированными $\left.Q_{i}, i=0,1,2\right)$. Ее производная есть

$$
\frac{\partial \mathscr{D}}{\partial V}=2 a\left[Q_{0} V-Q_{1}\right]
$$

Ввиду (3.52) и (3.53) сушествуют положительные постоянные $0<E_{1}<E_{2}$ такие, что для всех $t<t_{*}$

$$
-E_{2}<\frac{\partial \mathscr{D}}{\partial V}<-E_{1}
$$

Следовательно, по теореме о среднем значении для всех $t<t_{*}$

$$
-E_{2}<\frac{\mathscr{D}(t)}{V(t)-V_{0}(t)}<-E_{1}
$$

Теперь мы докажем (3.45) для всех $t<t_{*}$ с некоторой постоянной $C_{0}>0$, не зависяшей от выбора $B$ в (3.44). Напомним, что мы положили начальную скорость поршня $V(0)=W(0)$ и что $W(0)=V_{0}(0)$, см. $\S 2$. Теперь, от противного, пусть $t \in\left(0, t_{*}\right)-$ время, при котором (3.45) нарушается впервые. Обозначим через

$$
\Delta_{0}=\frac{1}{L^{3 / 2} \ln L}
$$

максимально допустимое приращение времени в теореме 3.4. Пусть $s=t-\Delta_{0}$. Из теоремы 3.4 следует, что

$$
V(t)=V(s)+\mathscr{D}(s) \Delta_{0}+\chi
$$


где

$$
|\chi| \leqslant \frac{C \sqrt{\Delta_{0}} \ln L}{L}=\frac{C \sqrt{\ln L}}{L^{4 / 3}} .
$$

Из (3.58) получаем, что

$$
V_{0}(t)=V_{0}(s)+\chi_{0}
$$

$\mathrm{c}$

$$
\left|\chi_{0}\right| \leqslant \frac{E_{0} \varepsilon_{0} \Delta_{0}}{L}=\frac{E_{0} \varepsilon_{0}}{L^{5 / 3} \ln L} .
$$

Для краткости положим $U(s)=V(s)-V_{0}(s)$ для всех $s$. Вычитая (3.61) из (3.60), получим

$$
U(t)=U(s)+\mathscr{D}(s) \Delta_{0}+\chi^{\prime}
$$

с $\chi^{\prime}=\chi-\chi_{0}$, так что для больших $L$

$$
\left|\chi^{\prime}\right| \leqslant \frac{2 C \sqrt{\ln L}}{L^{4 / 3}}
$$

Предположим без потери обшности, что $U(t)>0$. Так как $(3.45)$ не вьполняется в момент времени $t$, мы имеем

$$
U(t) \geqslant \frac{C_{0}(\ln L)^{3 / 2}}{L^{2 / 3}}
$$

Рассмотрим два случая. Если $U(s) \leqslant 0$, тогда в силу $(3.59)$

$$
U(t) \leqslant|\mathscr{D}(s)| \Delta_{0}+\left|\chi^{\prime}\right| \leqslant E_{2}|U(s)| \Delta_{0}+\left|\chi^{\prime}\right| \ll \frac{(\ln L)^{3 / 2}}{L^{2 / 3}}
$$

для больших $L$, что противоречит (3.64). Если $U(s)>0$, тогда снова из (3.62) и (3.59) получаем

$$
U(t)<U(s)\left[1-E_{1} \Delta_{0}\right]+\chi^{\prime}
$$

следовательно,

$$
U(s)>\frac{U(t)-\chi^{\prime}}{1-E_{1} \Delta_{0}}>\left(U(t)-\chi^{\prime}\right)\left(1+E_{1} \Delta_{0}\right)>U(t)+U(t) E_{1} \Delta_{0}-2 \chi^{\prime} .
$$

Теперь если $C_{0}$ в $(3.45)$ достаточно велико, скажем, $C_{0}=5 C / E_{1}$, то $U(t) E_{1} \Delta_{0}>2 \chi^{\prime}$ по (3.64) и (3.63). Из этого факта и из (3.65) следует $U(s)>U(t)$, так что (3.45) нарушается в более ранний момент времени $s<t$, что противоречит сделанному предположению. Таким образом, (3.45) доказано для всех $t<t_{*}$ и $C_{0}=5 C / E_{1}$.

Комбинируя (3.54) с (3.45), получим для больших $L$ и для всех $t<t_{*}$

$$
|d X(t) / d t|<2 C_{3} \varepsilon_{0}+C_{4} L^{-1}|X(t)-L / 2| .
$$

Используя стандартное неравенство Гронуолла из теории дифференциальных уравнений (см., например, лемму 2.1 в [26]), получим

$$
|X(t)-L / 2|<2 \varepsilon_{0} C_{3} C_{4}^{-1} L\left(e^{C_{4} L^{-1} t}-1\right)
$$


и

$$
|V(t)|<2 \varepsilon_{0} C_{3} e^{C_{4} L^{-1} t}
$$

для всех $t<t_{*}$. Из $(3.8)$

$$
|V(t)|<2 \varepsilon_{0} C_{3} e^{C_{4} v_{\max }^{-1}}
$$

Теперь мы выбираем $B=3 C_{3} e^{C_{4} v_{\max }^{-1}}$. Ясно, что тогда (3.66) будет противоречить (3.48). Таким образом завершается доказательство (3.44) для всех $t<S_{1}$. Теорема 3.5 теперь доказана.

СлЕДСТВИЕ 3.6. Предположим, что $\varepsilon_{0}>0$ в (1.11) достаточно мало. Тогда для всех больиих $L$ и всех $\omega \in \Omega_{0}^{*}$ имеем $S_{1}=T_{1}$, т.е. предыдущие теоремь справедливь для целого интервала без перестолкновений $\left(0, T_{1}\right)$.

ДокАЗАТЕльство. Напомним, что “медленньй” интервал $\left(0, S_{1}\right) \subset\left(0, T_{1}\right)$ определяется двумя условиями (a) и (b). Если $S_{1}<T_{1}$, то или (a), или (b) не выполняется в $S_{1}$. Ясно, что (а) не может перестать вьполняться "резко", так как (3.44) справедливо для всех $t<S_{1}$.

Предположим, что (b) нарушается при некотором $s<T_{1}$, в то время как (a) еще выполнено. Нарушение условия (b) означает, что в момент времени $s$ поршень "сталкивается" с точкой $(x, v)$ такой, что $v_{\min }<|v|<v_{\max }$ и $(x, v) \notin G_{0}(s)$. Следовательно, обрашенная траектория $F^{-(s-t)}(x, v), 0<t<s$, точки $(x, v)$ ударяется о поршень в некоторьй момент времени $t>0$. В течение интервала времени $(0, t)$ поршень проходит расстояние $|X(t)-L / 2| \leqslant B \varepsilon_{0} t$, а в интервале времени $(t, s)$ наша точка проходит расстояние $|v|(s-t)<v_{\max }(s-t)$. Следовательно,

$$
\begin{aligned}
L & \leqslant|X(t)-L / 2|+|v|(s-t)+|X(s)-L / 2| \\
& \leqslant|X(s)-L / 2|+v_{\max } s-\left(v_{\max }-B \varepsilon_{0}\right) t .
\end{aligned}
$$

С другой стороны, так как $s<T_{1}$, мы имеем

$$
L>|X(s)-L / 2|+v_{\max } s
$$

см. доказательство леммы 3.2. Это противоречие показьвает, что (b) не может не вьполняться. Доказательство следствия 3.6 закончено.

ЗАмЕчАНИЕ. Мы здесь положили начальную скорость поршня $V(0)=W(0)$, в то время как в $\S 1$ она была равна 0 . Если $V(0)=0$, тогда из теоремы 3.4 следовало бы, что $V(t)$ сходится к $V_{0}(t)$ экспоненциально быстро по $t$, пока она остается $\delta$-близкой к $V_{0}(t)$ с $\delta=C_{0}(\ln L)^{3 / 2} L^{-2 / 3}$. С учетом этого факта все наши результаты будут применимы без изменений. Начальньй интервал, на котором эта сходимость имеет место, будет порядка $\ln L$, а в гидродинамическом времени он есть $L^{-1} \ln L$, что исчезает при $L \rightarrow \infty$. Вот почему мы просто для наибольшего удобства положили здесь $V(0)=W(0)$.

Следуюшая теорема улучшает результаты теорем 3.4 и 3.5 . 
Теорема 3.7. Предположим, что $\varepsilon_{0}>0$ в (1.11) достаточно мало. Тогда существует такая постоянная $C>0$, что для всех больших $L$, всех $\omega \in \Omega_{0}^{*} u$ всех $t<T_{1}$

$$
\left|V(t)-V_{0}(t)\right|<\frac{C \ln L}{L}
$$

и для любого интервала времени $(t, t+\Delta t) \subset\left(0, T_{1}\right)$ такого, что $L^{-2}<\Delta t \leqslant 1$,

$$
|V(t+\Delta t)-V(t)|<C \frac{\ln L \sqrt{\Delta t}}{L} .
$$

ДоКАЗАТЕЛЬСТвО. Из нашего выбора начальной скорости $V(0)=V_{0}(0)$ прямо следует (3.67), по крайней мере, для маленьких $t$. Предположим, что (3.67) нарушается при некотором $t_{*}<T_{1}$ и $t_{*}-$ наименьшее время такого нарушения. Не теряя обшности, предположим, что

$$
V\left(t_{*}\right)-V_{0}\left(t_{*}\right) \geqslant \frac{C \ln L}{L} .
$$

Пусть $0<t_{0}<t_{*}-$ наибольшее время, при котором

$$
V\left(t_{0}\right)-V_{0}\left(t_{0}\right)<\frac{C \ln L}{2 L} .
$$

Тогда для всех $t \in\left(t_{0}, t_{*}\right)$

$$
V\left(t_{0}\right)-V_{0}\left(t_{0}\right) \leqslant V(t)-V_{0}(t) \leqslant V\left(t_{*}\right)-V_{0}\left(t_{*}\right) .
$$

Пусть

$$
\Delta t=\min \left\{1, t_{*}-t_{0}\right\} .
$$

Мы будем изучать динамику поршня в интервале времени $\left(t_{0}, t_{0}+\Delta t\right)$. Прямо из (3.58) мы имеем

$$
\left|V_{0}(t)-V_{0}\left(t_{0}\right)\right|<\delta V:=E_{0} \varepsilon_{0} L^{-1} \Delta t
$$

для всех $t \in\left(t_{0}, t_{0}+\Delta t\right)$. Следовательно, из (3.71) вытекает

$$
V(t)>V\left(t_{0}\right)-\delta V .
$$

Заметим, что $\Delta t$ не очень мало, по крайней мере, $\Delta t>\left(L^{2 / 3} \ln L\right)^{-1}$. В самом деле, если бы это было не так, то, положив $t_{*}=t_{0}+\Delta t$, мы из (3.69), (3.70) и (3.73) заключили бы, что

$$
V\left(t_{*}\right)-V\left(t_{0}\right) \geqslant 2^{-1} C L^{-1} \ln L-E_{0} \varepsilon_{0} L^{-5 / 3} .
$$

Последнее противоречило бы теореме 3.4 , так как $\mathscr{D}\left(t_{0}\right)<0$ (потому что $V\left(t_{0}\right)>$ $\left.V_{0}\left(t_{0}\right)\right)$. 
Теперь мы разовьем обобшенную версию разложения скорости (3.27) в теореме 3.4 . Мы разобьем интервал $\left(t_{0}, t_{0}+\Delta t\right)$ на подьнтервалы длины $\delta$ с концами $t_{i}=t_{0}+i \delta$, $i=0,1, \ldots, I$, где $I=\Delta t / \delta$. Мы выберем $\delta$ так, что

$$
\frac{0.5}{L \ln L}<\delta<\frac{1}{L \ln L}
$$

и $\Delta t / \delta$ - целое (для удобства). Предварительные требования в доказательстве теоремы 3.4 позволяют нам подобрать время так, что $L^{2} t_{0}$ и $L^{2} \delta$ будут цельми, поэтому $L^{2} t_{i}$ будет целым для каждого $i$. Теперь применим к каждому подынтервалу $\left(t_{i}, t_{i+1}\right)$ длины $\delta$ разложение скорости из доказательства теоремы 3.4. В частности, из (3.27) следует, что

$$
V\left(t_{i+1}\right)-V\left(t_{i}\right)=-\varepsilon k_{i} V\left(t_{i}\right)+\varepsilon \sum_{j=1}^{k_{i}} v_{j}+\chi_{i}^{(1)},
$$

где $k_{i}$ - число частиц, сталкивающихся с поршнем в интервале времени $\left(t_{i}, t_{i+1}\right)$, и $v_{j}, 1 \leqslant j \leqslant k_{i}$, - их скорости. Флуктуационный член $\chi_{i}^{(1)}$ может быть ограничен выражением из (3.32):

$$
\left|\chi_{i}^{(1)}\right| \leqslant 8 a^{2} v_{\max }^{3}(\ln L)^{2} \delta^{2} .
$$

Благодаря (3.74) разложение (3.76) может быть переписано как

$$
V\left(t_{i+1}\right)-V\left(t_{i}\right) \leqslant-\varepsilon k_{i} V\left(t_{0}\right)+\varepsilon \sum_{j=1}^{k_{i}} v_{j}+\chi_{i}^{(1)}+\chi_{i}^{(2)}
$$

$\mathrm{c}$

$$
\left|\chi_{i}^{(2)}\right| \leqslant \varepsilon k_{i} \delta V \leqslant 2 a v_{\max } E_{0} \varepsilon_{0} L^{-1} \ln L \Delta t \delta,
$$

где на последнем шаге мы использовали (3.31) и (3.73). Суммирование (3.78) по $i$ дает

$$
V\left(t_{0}+\Delta t\right)-V\left(t_{0}\right) \leqslant-\varepsilon k V\left(t_{0}\right)+\varepsilon \sum_{j=1}^{k} v_{j}+\chi^{(3)},
$$

где $k$ - число частиц, сталкиваюшихся с поршнем в интервале времени $\left(t_{0}, t_{0}+\Delta t\right)$, $v_{j}, 1 \leqslant j \leqslant k,-$ их скорости и

$$
\begin{aligned}
\left|\chi^{(3)}\right| & \leqslant 8 a^{2} v_{\max }^{3}(\ln L)^{2} \delta \Delta t+2 a v_{\max } E_{0} \varepsilon_{0} L^{-1} \ln L(\Delta t)^{2} \\
& \leqslant\left(8 a^{2} v_{\max }^{3}+2 a v_{\max } E_{0} \varepsilon_{0}\right) L^{-1} \ln L(\Delta t)
\end{aligned}
$$

(здесь мы использовали (3.73) и предположение $\Delta t \leqslant 1$ ). Разложение (3.79) может быть проанализировано подобно (3.27) в доказательстве теоремы 3.4. Определим на $(x, v)$-плоскости область

$$
D_{1}=\left\{(x, v): \frac{v-V\left(t_{0}\right)+\delta V}{x-X\left(t_{0}\right)}<-\frac{1}{\Delta t}, v_{\min }<|v|<v_{\max }\right\} .
$$

Она является объединением двух трапеций: $D_{1}=D_{1}^{+} \cup D_{1}^{-}$, где $D_{1}^{-}$обозначает верхнюю и $D_{1}^{+}$- нижнюю трапецию, см. рис. 4 . Изграницы (3.74) следует, что все частицы 
в области $D_{1}^{+}$обязательно сталкиваются с поршнем в интервале времени $\left(t_{0}, t_{0}+\Delta t\right)$, а все частищы, действительно сталкиваюшиеся с поршнем слева от него в том же интервале времени, содержатся в $D_{1}^{-}$.

Так как $v>V\left(t_{0}\right)$ для всех частиц $(x, v) \in D_{1}^{-}$и $v<V\left(t_{0}\right)$ для всех $(x, v) \in D_{1}^{+}$, мы можем отбросить из (3.79) частищы, не принадлежащие к $D_{1}^{+}$, и одновременно добавить к (3.79) частицы, принадлежашие к $D_{1}^{-}$, но не сталкивающиеся с поршнем. Эта процедура только увеличивает правую часть в (3.79), следовательно,

$$
V\left(t_{0}+\Delta t\right)-V\left(t_{0}\right) \leqslant-\varepsilon k_{1} V\left(t_{0}\right)+\varepsilon \sum_{j=1}^{k_{1}} v_{j}+\chi^{(3)},
$$

где $k_{1}$ - число частищ в $D_{1}$ в момент времени $t_{0}$ и суммирование проводится по всем таким частицам. Пусть $Z_{1}=\sum_{(x, v) \in D_{1}} v$. Точно так же, как в доказательстве теоремы 3.4, наши главные требования здесь гарантируют, что

$$
\left|k_{1}-\mathrm{E}\left(k_{1}\right)\right| \leqslant c_{3} L \ln L \sqrt{\Delta t}
$$

и

$$
\left|Z_{1}-\mathrm{E}\left(Z_{1}\right)\right| \leqslant c_{4} L \ln L \sqrt{\Delta t}
$$

для некоторых постоянных $c_{3}, c_{4}>0$, не зависяших от выбора $C$ в $(3.67)$, которьй мы еще не сделали. Теперь, вычисляя средние значения величин $k_{1}$ и $Z_{1}$, как и в доказательстве теоремы 3.4, мы приходим к неравенству

$$
V\left(t_{0}+\Delta t\right)-V\left(t_{0}\right) \leqslant \mathscr{D}\left(t_{0}\right) \Delta t+\chi^{(3)}+\chi^{(4)}
$$

$\mathrm{c}$

$$
\left|\chi^{(4)}\right| \leqslant a\left(c_{3}+c_{4}\right) L^{-1} \ln L \sqrt{\Delta t}+c_{5} \delta V \Delta t,
$$

где $c_{5}>0$ - постоянная, не зависящая от выбора $C$ в (3.67). Последний член в $(3.84)$ возникает благодаря добавке $\delta V$ к скорости $V\left(t_{0}\right)$ в $(3.81)$. Этот последний член не превосходит $c_{5} E_{0} \varepsilon_{0} L^{-1}(\Delta t)^{2}$ и, так как $\Delta t \leqslant 1$, может быть включен в первьй член из (3.84). Напомним, что $V\left(t_{0}\right)-V_{0}\left(t_{0}\right) \approx 2^{-1} C L^{-1} \ln L>0$ (здесь мы имеем приближение с точностью до величины порядка $1 / L^{2}$, так как скорость поршня при каждом столкновении изменяется на $\left.O\left(1 / L^{2}\right)\right)$. Тогда прямо из $(3.59)$ мы имеем

$$
\mathscr{D}\left(t_{0}\right) \leqslant-E_{1}\left(V\left(t_{0}\right)-V_{0}\left(t_{0}\right)\right) \approx 2^{-1} C E_{1} L^{-1} \ln L .
$$

Таким образом, комбинирование полученных выше оценок дает

$$
V\left(t_{0}+\Delta t\right)-V\left(t_{0}\right) \leqslant 2^{-1} C E_{1} L^{-1} \ln L \Delta t+\chi^{(5)}
$$

c $\chi^{(5)}=\chi^{(3)}+\chi^{(4)}$, ограниченным в силу $(3.80)$ и (3.84):

$$
\left|\chi^{(5)}\right| \leqslant c_{6}\left(L^{-1} \ln L \Delta t+L^{-1} \ln L \sqrt{\Delta t}\right),
$$

где постоянная $c_{6}>0$ не зависит от выбора $C$ в (3.67). Теперь мы выбираем постоянную $C$ :

$$
C=\max \left\{c_{6}, 6 E_{1}^{-1} c_{6}\right\}
$$


Тогда из (3.85) вытекает, что

$$
V\left(t_{0}+\Delta t\right)-V\left(t_{0}\right) \leqslant c_{6} L^{-1} \ln L(-2 \Delta t+\sqrt{\Delta t})
$$

и, следовательно, из (3.73)

$V\left(t_{0}+\Delta t\right)-V_{0}\left(t_{0}+\Delta t\right) \leqslant V\left(t_{0}\right)-V_{0}\left(t_{0}\right)+c_{6} L^{-1} \ln L(-2 \Delta t+\sqrt{\Delta t})+E_{0} \varepsilon_{0} L^{-1} \Delta t$.

Мы имеем теперь два случая. Первьй: $t_{*}-t_{0} \leqslant 1$, следовательно, $\Delta t \leqslant 1$. Выражение $-2 \Delta t+\sqrt{\Delta t}$ имеет максимум, равный $1 / 8$, в точке $\Delta t=1 / 16$. Тогда из $(3.87)$ вытекает

$$
V\left(t_{*}\right)-V_{0}\left(t_{*}\right)<V\left(t_{0}\right)-V_{0}\left(t_{0}\right)+8^{-1} c_{6} L^{-1} \ln L+E_{0} \varepsilon_{0} L^{-1} .
$$

Это противоречит (3.69) и (3.70), когда $L$ достаточно велико (вспомним наш выбор $C$ в $(3.86))$. Рассмотрим второй случай: $t_{*}-t_{0}>1$. Тогда $\Delta t=1$ и для больших $L$ из (3.87) следует:

$$
V\left(t_{0}+1\right)-V_{0}\left(t_{0}+1\right)<V\left(t_{0}\right)-V_{0}\left(t_{0}\right)-2^{-1} c_{6} L^{-1} \ln L,
$$

что противоречит (3.71). Этим завершается доказательство (3.67).

Теперь докажем (3.68). Если $\Delta t<\left(L^{2 / 3} \ln L\right)^{-1}$, то мы можем из теоремы 3.4 получить:

$$
|V(t+\Delta t)-V(t)| \leqslant|\mathscr{D}(t)| \Delta t+C L^{-1} \ln L \sqrt{\Delta t} .
$$

Из полученных ранее оценок (3.59) и (3.67) вытекает, что

$$
|\mathscr{D}(t)| \leqslant E_{2}\left|V(t)-V_{0}(t)\right| \leqslant C E_{2} L^{-1} \ln L
$$

откуда следует (3.68) (с несколько большей постоянной $C$, чем выше).

Теперь пусть $\left(L^{2 / 3} \ln L\right)^{-1} \leqslant \Delta t \leqslant 1$. Без потери обшности предположим, что $V(t+\Delta t)>V(t)$. Кроме того, мы можем предположить, что

$$
V(s)>V(t) \text { для } s \in(t, t+\Delta t) \text {. }
$$

Если же это не так, мы можем заменить $t$ на $t^{\prime}=\max \{s<t+\Delta: V(s) \leqslant V(t)\}$ и доказать (3.68) для меньшего интервала $\left(t^{\prime}, t+\Delta t\right)$.

Далее наш план состоит в том, чтобы применить некоторые оценки из доказательства неравенства (3.67) и затем следовать доказательству теоремы 3.4 . Положим $t_{0}=t$ и разобьем интервал $\left(t_{0}, t_{0}+\Delta t\right)$ на подьштервалы длины $\delta$, удовлетворяюшие $(3.75)$. Тогда снова мы имеем разложения (3.76) и (3.77). Из (3.89) мы имеем $V\left(t_{i}\right)>V\left(t_{0}\right)$ для всех $i$; следовательно, из (3.76) вытекает, что

$$
V\left(t_{i+1}\right)-V\left(t_{i}\right) \leqslant-\varepsilon k_{i} V\left(t_{0}\right)+\varepsilon \sum_{j=1}^{k_{i}} v_{j}+\chi_{i}^{(1)} .
$$


Суммирование этих неравенств по $i$ дает (3.79) с границей (3.80), в которой второй член может быть просто отброшен, так как $\chi_{i}^{(2)}$ отсутствует. Далее, возможные флуктуации скорости поршня $V(s)$ в интервале времени $\left(t_{0}, t_{0}+\Delta t\right)$ могут быть оценены с помошью (3.67) и (3.73):

$$
\left|V(s)-V\left(t_{0}\right)\right| \leqslant \delta V:=2 C L^{-1} \ln L+E_{0} \varepsilon_{0} L^{-1} \Delta t
$$

для всех $s \in\left(t_{0}, t_{0}+\Delta t\right)$. Затем мы оцениваем случайные величины $k$ и $Z=\sum_{j} v_{j}$, как и в доказательстве теоремы 3.4 , начиная с построения двух областей $D_{1}$ и $D_{2}$ с помощью (3.29) и (3.30) и т. п. Повторяя почти дословно аргументы из доказательства теоремы 3.4, мы приходим к аналогу (3.41):

$$
V(t+\Delta t)-V(t)<\mathscr{D}(t) \Delta t+\chi^{\prime}
$$

с оценкой

$$
\left|\chi^{\prime}\right| \leqslant \text { const } \cdot\left[L^{-1} \ln L \sqrt{\Delta t}+\delta V \Delta t+L^{-1} \ln L \Delta t\right],
$$

вместо (3.32), где последний член возникает из (3.80) (отметим, что (3.32) не было бы более достаточным, так как $\Delta$ велико - вот почему нам было нужно разбить интервал $(t, t+\Delta t)$ на меньшие интервалы). Мы комбинируем полученные оценки с (3.88), чем завершаем доказательство (3.68) и теоремы 3.7 .

Наконец, мы доказьваем сходимость случайных траекторий поршня при $L \rightarrow \infty$ к решению $Y(\tau), W(\tau)$ гидродинамических уравнений из $§ 2$.

Теорема 3.8. Предположим, что $\varepsilon_{0}>0$ в (1.11) достаточно мало. Тогда для всех больших $L$ и всех $\omega \in \Omega_{0}^{*}$ существует постоянная $C>0$ такая, что

$$
\begin{aligned}
\left|Y_{L}(\tau, \omega)-Y(\tau)\right| & \leqslant \frac{C \ln L}{L}, \\
\left|W_{L}(\tau, \omega)-W(\tau)\right| & \leqslant \frac{C \ln L}{L}
\end{aligned}
$$

для всех $0<\tau<\min \left\{\tau_{1}, T_{1} / L\right\}$ u

$$
\left|\tau_{1}-T_{1} / L\right| \leqslant \frac{C \ln L}{L} .
$$

ДокАЗАТЕЛЬСТво. В $\S 2$ мы определили функцию $F(Y, \tau)$ так, что гидродинамическое решение $Y(\tau)$ удовлетворяет уравнению

$$
d Y(\tau) / d \tau=F(Y, \tau), \quad Y(0)=1 / 2
$$

(см. (2.29)). Из теоремы 3.7 следует, что для всех $\omega \in \Omega_{0}^{*}$ случайная траектория удовлетворяет уравнению

$$
\partial Y_{L}(\tau, \omega) / \partial \tau=F(Y, \tau)+\chi(\tau, \omega), \quad Y_{L}(0, \omega)=1 / 2,
$$


где

$$
|\chi(\tau, \omega)| \leqslant \frac{C \ln L}{L} .
$$

Вспомним, что $|\partial F(Y, \tau) / \partial Y| \leqslant \kappa\left(\right.$ см. (2.30)). Поэтому разность $Z_{L}(\tau, \omega):=Y_{L}(\tau, \omega)$ - $Y(\tau)$ удовлетворяет неравенству

$$
\left|Z_{L}^{\prime}(\tau, \omega)\right| \leqslant \kappa\left|Z_{L}(\tau, \omega)\right|+\frac{C \ln L}{L}
$$

и $Z_{L}(0, \omega)=0$. С помошю стандартного неравенства Гронуолла из теории дифференщиальных уравнений (см., например, лемму 2.1 в [26]) мы имеем

$$
\begin{aligned}
& \left|Z_{L}(\tau, \omega)\right| \leqslant \frac{C \ln L}{\kappa L}\left(e^{\kappa \tau}-1\right), \\
& \left|Z_{L}^{\prime}(\tau, \omega)\right| \leqslant \frac{C \ln L}{L} e^{\kappa \tau}
\end{aligned}
$$

для всех $\tau<\min \left\{\tau_{1}, T_{1} / L\right\}$, откуда следует (3.90) и (3.91).

Наконец, мы проверяем (3.92). Согласно (3.91) случайные флуктуации скорости поршня ограничены величиной $C L^{-1} \ln L$. Значит, случайные флуктуации скоростей частиц, которые сталкивались с поршнем один раз, ограниченывеличиной $2 C L^{-1} \ln L$. Случайные фллутуации положений и поршня и частиц в каждый момент времени $t<\min \left\{\tau_{1} L, T_{1}\right\}$ ограничены теми же величинами (быть может, сотличньм значением постоянной $C$ ) по координате $y=x / L$. С другой стороны, относительная скорость поршня и частиц отделена от нуля (скажем, $v_{\min }-4 B \varepsilon_{0}>0$ ). Следовательно, время первого перестолкновения $T_{1} / L$ может отличаться от $\tau_{1}$, самое большее, на const $\cdot L^{-1} \ln L$. Теорема 3.8 доказана.

\section{§ 4. Динамика меж,ду первым и вторым перестолкновениями}

В этом параграфе мы изучаем интервал $\left(\tau_{1}, \tau_{2}\right)$ с одним перестолкновением, в котором частицы газа испытьвают второе столкновение (т.е. первое перестолкновение) с поршнем.

Частицы, которые столкнулись с поршнем, не образуют больше пуассоновского процесса, поэтому их распределение значительно труднее контролировать. В этом заключается наша главная трудность. С другой стороны, нам будет достаточно значительно более слабых оценок, чем полученные в предыдущем параграфе. Кроме того, многие аргументы и построения в этом параграфе аналогичны тем, что были в $\S 3$, и мы опускаем некоторые детали. Мы сконцентрируемся на новых идеях.

Начиная с этого места и далее наш анализ будет ограничен конфигурациями $\omega \in \Omega_{0}^{*}$. Позже мы наложим дополнительные ограничения на $\omega$.

Напомним, что для $\omega \in \Omega_{0}^{*}$ скорость поршня мала, $|V(t)|<B \varepsilon_{0}$ (см. (3.44)), на интервале $\left(0, T_{1}\right)$ без перестолкновений. Следовательно, скорости частиц газа, испытьваюших одно столкновение с поршнем в интервале $\left(0, T_{1}\right)$, ограничены:

$$
v_{1, \min }<|v|<v_{1, \max }
$$

где

$$
v_{1, \min }:=v_{\min }-2 B \varepsilon_{0} \quad \text { и } \quad v_{1, \max }:=v_{\max }+2 B \varepsilon_{0} .
$$


Наименьшее время второго столкновения $T_{2}=T_{2}(\omega)$ определяется равенством: $T_{2}=$ $\sup _{t>0}\left\{G_{3}^{+}=\varnothing\right\}$ (см. (3.7)). Из (3.44) и (4.1), как в доказательстве леммы 3.2, может быть легко получена следующая оценка:

$$
T_{2} \leqslant \frac{L}{v_{\max }}+\frac{L}{v_{\max }-2 B \varepsilon_{0}} \leqslant \frac{3 L}{v_{\max }} .
$$

Пусть $(x, v) \in G^{+}$и $\left(x_{t}, v_{t}\right)=F^{t}(x, v)$ для $t>0$. Обозначим через

$$
s_{1}(x, v)=\min \left\{t: x_{t}=X(t)\right\}
$$

время первого столкновения с поршнем. Область

$$
G_{*}^{+}(t):=\left\{\left(x_{t}, v_{t}\right) \in G_{1}^{+}(t): s_{1}(x, v)<T_{1}\right\}
$$

занята точками, испытавшими к моменту времени $t$ одно столкновение с поршнем, которое случилось прежде $T_{1}$. Опустив верхний индекс + в предыдущих формулах, мы определяем $G_{*}(t)$. Пусть $T_{*} \leqslant T_{2}-$ самьй ранний момент времени, в которьй поршень взаимодействует с частицами

$$
(x, v) \in\left[G_{1}^{+}(t) \backslash G_{*}^{+}(t)\right] \cup G_{2}^{+}(t) .
$$

Время $T_{*}$ является случайным аналогом времени $\tau_{*}$, введенного в $\S 2$. В интервале $\left(T_{1}, T_{*}\right)$ поршень взаимодействует с частицами из $G_{0}^{+}(t) \cup G_{*}^{+}(t)$, поэтому их скорости должны быть ограничены неравенством (4.1). Обозначим через

$$
\begin{aligned}
\mathscr{X}_{1}(t)= & \left\{(x, v): x=X(t)+0,-v_{1, \max }<v<-v_{1, \min }\right\} \\
& \cup\left\{(x, v): x=X(t)-0, v_{1, \min }<v<v_{1, \max }\right\}
\end{aligned}
$$

объединение двух односторонних окрестностей, непосредственно примькающих к поршню, которое содержит все "входяшие" частицы для каждого $t \in\left(T_{1}, T_{*}\right)$.

Снова, как и в предыдушем параграфе, мы определяем подьнтервал $\left(T_{1}, S_{2}\right) \subset$ $\left(T_{1}, T_{*}\right)$, в котором поршень движется достаточно медленно.

ОПРЕДЕЛЕНИЕ. Пусть $\left(T_{1}, S_{2}\right) \subset\left(T_{1}, T_{*}\right)$ - максимальньй интервал времени, в котором:

(a) $|V(t)|<v_{1, \min }$;

(b) $\mathscr{X}_{1}(t) \subset G_{0}(t) \cup G_{*}(t)$.

Условие (b) означает, что частишы со скоростями $v_{1, \min }<|v|<v_{1, \max }$, которые близки к столкновению с поршнем в момент времени $t$, провзаимодействовали с ним в интервале $(0, t)$ самое большее один раз и это взаимодействие, если оно произошло, случилось прежде $T_{1}$.

Далее, мы оцениваем, как велик интервал $\left(T_{1}, S_{2}\right)$. Предположим, что

$$
|V(t)-W(t / L)| \leqslant \Delta
$$

для всех $t<S_{2}$ и некоторого малого $\Delta$ (мы позже оценим $\Delta$ и покажем, что $\Delta \rightarrow 0$ при $L \rightarrow \infty)$. Согласно (2.26) из этого непосредственно вытекает, что $|V(t)| \leqslant \Delta+\mathscr{B} \varepsilon_{0}$. Интегрирование (4.5) по $t$ дает

$$
|X(t)-L Y(t / L)| \leqslant t \Delta
$$

и, следовательно,

$$
|X(t)-L / 2| \leqslant\left(\Delta+\mathscr{B} \varepsilon_{0}\right) t .
$$


ПРЕДЛОЖЕНИЕ 4.1. Если (4.5) справедливо для $t<S_{2}$ при некотором малом $\Delta>0$, тогда $T_{2}-S_{2} \leqslant C L\left(\Delta+\varepsilon_{0}\right)$, где $C>0-$ постоянная.

ДокАЗАТЕЛЬство. Если $S_{2}=T_{2}$, утверждение тривиально. Если $S_{2}<T_{2}$, тогда либо $S_{2}<T_{*}$ и, тем самым, хотя бы одно из условий (a) или (b) предыдущего определения не выполнено в момент $S_{2}$, либо $S_{2}=T_{*}<T_{2}$. Заметим, что условие (а) не может нарушиться резко, так как мы предполагаем, что $|V(t)| \leqslant \Delta+\mathscr{B} \varepsilon_{0}$ на $\left(0, S_{2}\right)$, т.е. $V(t)$ остается малым. Если (b) нарушается, тогда в момент времени $S_{2}$ поршень сталкивается с точкой $(x, v)$ такой, что $v_{1, \min } \leqslant|v| \leqslant v_{1, \max }$, и пройденная траектория $\left(x_{t}, v_{t}\right):=F^{t-S_{2}}(x, v), t \in\left(0, S_{2}\right)$, которой ударяется о поршень в некоторьй момент времени $t_{1} \geqslant T_{1}$. Если $S_{2}=T_{*}<T_{2}$, тогда в момент времени $S_{2}$ поршень испытывает перестолкновение с частицей газа $(x, v)$, траектория которой в прошлом $\left(x_{t}, v_{t}\right):=F^{t-S_{2}}(x, v)$ сталкивается с поршнем первый раз в некоторый момент времени $t_{1} \geqslant T_{1}$. В последнем случае в силу (4.5)

$$
|v| \leqslant v_{\max }+2\left|V\left(t_{1}\right)\right| \leqslant v_{\max }+2\left(\Delta+\mathscr{B} \varepsilon_{0}\right) .
$$

В любом из двух рассмотренных случаев траектория $\left(x_{t}, v_{t}\right)$ сталкивается с поршнем дважды - первый раз в момент времени $t_{1} \geqslant T_{1}$ и второй раз в момент времени $t_{2}=S_{2}$. Обозначим через $X_{1}, X_{2}$ положение поршня и через $V_{1}, V_{2}$ его скорости в моменты времени $t_{1}, t_{2}$ соответственно. Без потери обшности предположим, что наша траектория лежит справа от поршня. Заметим, что для скорости $|v|=\left|v_{t}\right|$ при $t_{1}<t<t_{2}$ выполнено неравенство:

$$
|v| \leqslant \max \left\{v_{1, \max }, v_{\max }+2\left(\Delta+\mathscr{B} \varepsilon_{0}\right)\right\} \leqslant v_{\max }+2\left(\Delta+B_{1} \varepsilon_{0}\right)
$$

с $B_{1}=\max \{B, \mathscr{B}\}$. Тогда из очевидного тождества

$$
|v|\left(t_{2}-t_{1}\right)=\left(L-X_{1}\right)+\left(L-X_{2}\right)
$$

следует в силу (4.6) и (4.7), что

$$
t_{2}-t_{1} \geqslant \frac{L-\left(\Delta+\mathscr{B} \varepsilon_{0}\right)\left(t_{1}+t_{2}\right)}{v_{\max }+2\left(\Delta+B_{1} \varepsilon_{0}\right)} .
$$

С другой стороны, рассмотрим частищу, которая испытывает самое первое перестолкновение с поршнем (это происходит в момент времени $T_{1}$ ). После этого столкновения частица приобретает скорость, для которой $\left|v\left(T_{1}+0\right)\right| \geqslant v_{\max }-2\left|V\left(T_{1}\right)\right|$. Следующее столкновение этой частицы с поршнем происходит после $T_{2}$. Поэтому

$$
\begin{aligned}
T_{2}-T_{1} & \leqslant \frac{L / 2+\left|X\left(T_{1}\right)-L / 2\right|+L / 2+\left|X\left(T_{2}\right)-L / 2\right|}{v_{\max }-2\left|V\left(T_{1}\right)\right|} \\
& \leqslant \frac{L+\left(\Delta+\mathscr{B} \varepsilon_{0}\right)\left(T_{1}+T_{2}\right)}{v_{\max }-2\left(\Delta+\mathscr{B} \varepsilon_{0}\right)} .
\end{aligned}
$$

Комбинируя найденные оценки, получим, что

$$
T_{2}-S_{2} \leqslant\left(T_{2}-T_{1}\right)-\left(t_{2}-t_{1}\right) \leqslant C L\left(\Delta+\varepsilon_{0}\right)
$$

с некоторым $C>0$, определяемым постоянными $\mathscr{B}$ и $B$. Предложение 4.1 доказано. 
Далее мы изучим динамику в интервале времени $\left(T_{1}, S_{2}\right)$. Мы снова определяем плотность сталкиваюшихся частиц "на поршне" $q(v, t)$ с помошњю уравнения $(3.9)$ и функции $Q_{i}(t), i=0,1,2$, с помошњю (3.10)-(3.12). Подчеркнем, что теперь, в отличие от предыдушего параграфа, плотность $p(x, v, t)$ существенно зависит от $\omega$ (по крайней мере, для $\left.(x, v) \in G_{*}^{+}(t)\right)$; следовательно, $q(v, t)$ и $Q_{i}(t)$ будут зависеть от $\omega$ не только через положение поршня $X(t)$, но также через плотность $p(x, v, t)$ окружаюших частиц.

В предыдущих параграф̆ах мы ввели также детерминистскую плотность $\widetilde{p}(x, v, t)$. Теперь мы можем определить соответствующую детерминистскую плотность “на поршне":

$$
\widetilde{q}(v, t)= \begin{cases}\widetilde{p}(X(t)+0, v, t) & \text { при } v<0, \\ \widetilde{p}(X(t)-0, v, t) & \text { при } v>0\end{cases}
$$

(ср. с $(2.17))$, и детерминистские функции $\widetilde{Q}_{i}(t), i=0,1,2$, с помошњю уравнений, подобных (3.10)-(3.12), но с “волной" над соответствующими функциями. Мы используем “волну”, чтобы отличать такие детерминистские функции от случайных.

Сравним случайные функции $p, q, Q_{i}$ с их детерминистскими аналогами на интервале $\left(T_{1}, S_{2}\right)$. Так как преобразования $F^{t}: G^{+} \rightarrow G^{+}(t)$ и $\widetilde{F}^{t}: \widetilde{G}^{+} \rightarrow \widetilde{G}^{+}(t)$ (напомним, что $\left.\widetilde{G}^{+}(t)=\widetilde{F}^{t}\left(G^{+}\right)\right)$являются обратимыми сохраняюшими меру отображениями, то таким же является отображение

$$
\Psi^{t}=\widetilde{F}^{t} \circ F^{-t}
$$

которое переводит $G^{+}(t)$ в $\widetilde{G}^{+}(t)$. Следующая лемма легко следует из теоремы 3.8 .

ЛЕмма 4.2. Пусть $t<S_{2} u \omega \in \Omega_{0}^{*}$. Для каждого $(x, v) \in G_{\infty}^{+}(t)$ положим $(\widetilde{x}, \widetilde{v})=\Psi^{t}(x, v)$. Тогда

$$
|x-\widetilde{x}| \leqslant C \ln L \quad u \quad|v-\widetilde{v}| \leqslant C L^{-1} \ln L
$$

с некоторой постоянной $C>0$. Мы также имеем

$$
p(x, v, t)=p(\widetilde{x}, \widetilde{v}, t) .
$$

Свойства плотности $p(x, v, t)$, сформулированные в лемме 3.3 , очевидным образом справедливы для детерминистской плотности $\widetilde{p}(x, v, t)$ во всей области $\widetilde{G}^{+}$. А именно, плотность $\widetilde{p}(x, v, t)$ является кусочно гладкой $C^{1}$-функцией с $|\partial \widetilde{p}(x, v, t) / \partial x| \leqslant D_{1}^{\prime} / L$ и линии разрьва плотности $\widetilde{p}(x, v, t)$ имеют наклон порядка $O(1 / L)$ (т.е. они почти параллельны оси $x)$. Таким образом, как в (3.13), мы имеем

$$
\left|\frac{\partial \widetilde{Q}_{i}}{\partial X}\right| \leqslant \frac{\text { const }}{L}
$$

Лемма 4.2 позволяет нам сравнивать $Q_{i}$ и $\widetilde{Q}_{i}$ как функции от $X$ следуюшим образом. 
Лемма 4.3. Пусть $t<S_{2} u \omega \in \Omega_{0}^{*}$. Тогда для $i=0,1,2$

$$
\left|Q_{i}(t)-\widetilde{Q}_{i}(t)\right| \leqslant C L^{-1} \ln L .
$$

Заметим, что $\widetilde{Q}_{i}(t)$ определяется через плотность $\widetilde{q}(v, t)$, которая использует (случайное) положение поршня $X(t)$ для данного $\omega($ см. (4.8)). Предыдушая лемма 4.2 показывает, что зависимость $Q_{i}(t)$ от $\omega$ через $p(x, v, t)$ (которая самазависит от $\omega$ ) очень слабая, так как $L^{-1} \ln L$ мало. Другими словами, плотность $p(x, v, t)$ газа, окружаюшего поршень, флуктуирует очень мало в зависимости от $\omega$. Следуюшая теорема является аналогом теоремы 3.4 .

Tеорема 4.4. Для всех достаточно больших L существует множество $\Omega_{1}^{*} \subset \Omega_{0}^{*}$ начальных конфигурачий частич такое, что

(i) существует такая постоянная $c>0$, что

$$
\mathrm{P}\left(\Omega_{1}^{*}\right)>1-L^{-c \ln \ln L} ;
$$

(ii) для каждой конфигурации $\omega \in \Omega_{1}^{*}$ и каждого интервала времени

$$
(t, t+\Delta t) \subset\left(T_{1}, S_{2}\right)
$$

такого, что

$$
\frac{(\ln L)^{2}}{L^{1 / 3}} \leqslant \Delta t \leqslant \frac{1}{L^{1 / 7}},
$$

изменение скорости поршня удовлетворяет равенству

$$
V(t+\Delta t)-V(t)=\widetilde{\mathscr{D}}(t) \Delta t+\chi,
$$

ฉde

$$
\widetilde{\mathscr{D}}(t)=a\left[\widetilde{Q}_{0}(t) V^{2}(t)-2 \widetilde{Q}_{1}(t) V(t)+\widetilde{Q}_{2}(t)\right]
$$

$u$

$$
|\chi| \leqslant C \frac{\ln L(\Delta t)^{1 / 4}}{L^{1 / 4}}
$$

с некоторой глобальной постоянной $C>0$.

ЗАмЕчАНИЕ. Отметим, что наша оценка (4.14) на случайные флуктуации, представленные в $\chi$, значительно слабее, чем (3.17) в теореме 3.4. Как станет ясным из доказательства, это связано с недостатком контроля за большими уклонениями для распределения частиц газа. 
ДокАЗАТЕльство. Наши аргументы в основном построены на идеях доказательства теоремы 3.4. Но они включаются в значительной степени в новую конструкцию, которую мы описьваем в деталях. Первый шаг состоит в схеме разложения скорости (cм. (3.27)):

$$
V(t+\Delta t)=(1-\varepsilon k) V(t)+\varepsilon \sum_{j=1}^{k} v_{j}+\chi^{(1)}+\chi^{(2)} .
$$

Поправки $\chi^{(1)}$ и $\chi^{(2)}$ определены в $\S 3$ после (3.27) и ограничены:

$$
\left|\chi^{u}\right| \leqslant v_{\max } \varepsilon^{2} k^{2}
$$

для $u=1,2$ (см. (3.32)). Мы увидим далее, что

$$
k \leqslant \text { const } \cdot L^{2} \Delta t
$$

и, следовательно,

$$
\left|\chi^{u}\right| \leqslant \text { const } \cdot(\Delta t)^{2}
$$

для $u=1,2$.

Нам нужна теперь грубая оценка сверху на возможные флуктуации $\delta V$ скорости поршня $V(s)$ в интервале $(t, t+\Delta t)$, как они были определены в (3.28). Можно легко вьвести из (4.15), что

$$
\delta V \leqslant 2 v_{\max } \varepsilon k \leqslant \text { const } \cdot \Delta t .
$$

Определим две области $D_{1}=D_{1}^{+} \cup D_{1}^{-}$и $D_{2}=D_{2}^{+} \cup D_{2}^{-}$на $(x, v)$-плоскости уравнениями $(3.29),(3.30)$, в которых $v_{\min }$ и $v_{\max }$ должны быть заменены на $v_{1, \min }$ и $v_{1, \max }$ соответственно. Как было объяснено в $\S 3$, все частишы из $D_{1}$ (в момент времени $t$ ) обязательно столкнутся с поршнем в интервале $(t, t+\Delta)$. А все частицы, действительно столкнувшиеся с поршнем в течение этого интервала времени, содержатся в $D_{2}$. Значит, мы имеем

$$
k_{1}^{ \pm} \leqslant k^{ \pm} \leqslant k_{2}^{ \pm}
$$

где $k^{ \pm}, k_{1}^{ \pm}, k_{2}^{ \pm}$определены в тексте, окружающем (3.34). Это дает верхнюю и нижнюю оценки для числа сталкивающихся частищ.

Наш следующий шаг состоит в оценке чисел $k_{i}^{ \pm}, i=1,2$. Так как распределения $k_{i}^{ \pm}$больше не являются пауссоновскими, мы оцениваем их, используя новый подход. Пусть $D$ - одна из четырех трапеций $D_{i}^{ \pm}, i=1,2$; и пусть $k_{D, \omega}-$ (случайное) число частиц в $D$ в момент времени $t$. Очевидно, $k_{D, \omega}$ равно числу частиц в $F^{-t}(D)$ в нулевой момент времени.

Отметим, что трапеция $D$ имеет высоту $v_{1, \max }-v_{1, \min }=$ const и ширину $O(\Delta t)$; значит, ее площадь ограничена:

$$
d_{1} \Delta t \leqslant|D| \leqslant d_{2} \Delta t
$$

где $0<d_{1}<d_{2}<\infty$-некоторые постоянные.

Изучим область $F^{-t}(D)$ более подробно. Так как $(t, t+\Delta t) \subset\left(T_{1}, S_{2}\right)$, из условия (b) определения $S_{2}$ следует, что $D \subset G_{0}(t) \cup G_{*}(t)$. Положим $D_{0}=D \cap G_{0}(t)$ 
и $D_{1}=D \cap G_{*}(t)$, тогда $F^{-1}(D)=F^{-1}\left(D_{0}\right) \cup F^{-1}\left(D_{1}\right)$. Чтобы подчеркнуть зависимость потока $F^{t}$ от $\omega$, будем писать $F_{\omega}^{t}$ вместо $F^{t}$. Часть $F_{\omega}^{-t}\left(D_{0}\right)$ получается простым линейньм преобразованием области $D_{0}$ (благодаря отсутствию столкновений с поршнем частиц из $D_{0}$ ), поэтому эта часть в действительности не зависит от $\omega$. Часть $F_{\omega}^{-t}\left(D_{1}\right)$ также получается переносом области $D_{1}$, по времени назад, но вдоль этого пути происходит одно столкновение с поршнем. Так как положение и скорость поршня случайны, область $F_{\omega}^{-t}(D)$ будет зависеть от $\omega$. Следовательно, область $D_{\omega}:=F_{\omega}^{-t}(D)$ будет зависеть от $\omega$. Начальное число частищ $k_{D, \omega}$ в случайно выбранной области $D_{\omega}$, конечно, не будет пуассоновской случайной величиной.

Для оценки $k_{D, \omega}$ мы зафиксируем некоторое $\Delta t$, удовлетворяющее (4.11), и построим конечное число областей $D_{n} \subset G, 1 \leqslant n \leqslant N_{*}$, обладающих следуюшим свойством. Для каждого $\omega \in \Omega_{0}^{*}$, каждого $t \in\left(T_{1}, S_{2}-\Delta t\right)$ и каждой трапеции $D$, определенной выше, сушествуют две области $D_{n^{\prime}}, D_{n^{\prime \prime}}$ такие, что

$$
D_{n^{\prime}} \subset F_{\omega}^{-t}(D) \subset D_{n^{\prime \prime}}
$$

и плошадь их разности относительно мала:

$$
\left|D_{n^{\prime \prime}} \backslash D_{n^{\prime}}\right| \leqslant \chi^{(3)}\left|D_{\omega}\right|,
$$

где $\chi^{(3)} \rightarrow 0$ при $L \rightarrow \infty$. Мы будем говорить, что $D_{n^{\prime}}$ и $D_{n^{\prime \prime}}$ аппроксимируют $D_{\omega}$ "изнутри" и "извне" соответственно. Для данного $\Delta t$ обозначим совокупность областей $D_{n}$ как

$$
\mathscr{C}=\mathscr{C}_{\Delta t}=\left\{D_{n}\right\}_{n=1}^{N_{*}} .
$$

Мы отложим на время построение областей $D_{n}$ и извлечем непосредственную пользу из указанных аппроксимаций. Из включения (4.21) вытекает, что

$$
k_{n^{\prime}, \omega} \leqslant k_{D, \omega} \leqslant k_{n^{\prime \prime}, \omega}
$$

где $k_{n, \omega}$ - (случайное) число частиц в области $D_{n}$ в нулевой момент времени. Так как $D_{n}$ для каждого $n$ фиксировано (не зависит от $\omega$ ), случайная величина $k_{n, \omega}$ имеет пуассоновское распределение со средним значением

$$
\lambda_{n}:=L^{2} \int_{D_{n}} p(x, v, 0) d x d v .
$$

Согласно лемме А.2

$$
\mathrm{P}\left(\omega:\left|k_{n, \omega}-\lambda_{n}\right|>B \sqrt{\lambda_{n}}\right) \leqslant 2 \varepsilon^{-c B^{2}}
$$

для любого $B<b \sqrt{\lambda_{n}}$, где $b>0$ - постоянная и $c=c(b)>0$ - другая постоянная. Значение $B=B_{\Delta t}$ (одно для всех $D_{n}$ из $\mathscr{C}_{\Delta t}$ ) мы выберем позднее. В силу (4.20) достаточно потребовать, чтобы

$$
B_{\Delta t}<L \sqrt{\Delta t} .
$$

Затем мы определяем $\Omega_{1}^{*}(\Delta t)$ как множество конфигураций $\omega \in \Omega_{0}^{*}$, удовлетворяющих неравенству

$$
\left|k_{n, \omega}-\lambda_{n}\right| \leqslant B_{\Delta t} \sqrt{\lambda_{n}} \text { для } 1 \leqslant n \leqslant N_{*} .
$$


Благодаря (4.24) получаем

$$
\mathrm{P}\left(\Omega_{0}^{*} \backslash \Omega_{1}^{*}(\Delta t)\right) \leqslant 2 N_{*} e^{-c B_{\Delta t}^{2}} .
$$

Из (4.23) и (4.26) для всех $\omega \in \Omega_{1}^{*}(\Delta t)$ следует

$$
\lambda_{n^{\prime}}-B_{\Delta t} \sqrt{\lambda_{n^{\prime}}} \leqslant k_{D, \omega} \leqslant \lambda_{n^{\prime \prime}}+B_{\Delta t} \sqrt{\lambda_{n^{\prime \prime}}} .
$$

Далее, рассмотрим величину

$$
\lambda_{D, \omega}=L^{2} \int_{F_{\omega}^{-1}(D)} p(x, v, 0) d x d v=L^{2} \int_{D} p(x, v, t) d x d v .
$$

Прямо из включений (4.21) мы получаем

$$
\lambda_{n^{\prime}} \leqslant \lambda_{D, \omega} \leqslant \lambda_{n^{\prime \prime}}
$$

Из (4.22) следует, что

$$
\left(1-c \chi^{(3)}\right) \lambda_{D, \omega} \leqslant \lambda_{n^{\prime}} \leqslant \lambda_{n^{\prime \prime}} \leqslant\left(1+c \chi^{(3)}\right) \lambda_{D, \omega}
$$

с некоторой постоянной $c>0$ (определяемой по $\pi_{\max }, \pi_{\min }, v_{1}$ и $v_{2}$ в $\left.(\mathrm{P} 3)\right)$.

Рассмотрим детерминированную величину

$$
\widetilde{\lambda}_{D}=L^{2} \int_{D} \widetilde{p}(x, v, t) d x d v .
$$

Из леммы 4.2 и свойств функции $\widetilde{p}(x, v, t)$ легко следует, что

$$
\left|\lambda_{D, \omega}-\widetilde{\lambda}_{D}\right| \leqslant C L^{-1} \ln L \widetilde{\lambda}_{D}
$$

для некоторой постоянной $C>0$. Комбинируя (4.29) и (4.30), мы приходим к соотношению

$$
\left(1-\chi^{(4)}\right) \widetilde{\lambda}_{D} \leqslant \lambda_{n^{\prime}} \leqslant \lambda_{n^{\prime \prime}} \leqslant\left(1+\chi^{(4)}\right) \widetilde{\lambda}_{D}
$$

c

$$
\chi^{(4)}=\mathrm{const} \cdot\left(\chi^{(3)}+L^{-1} \ln L\right) .
$$

Из (4.28) и (4.31) мы получим желаемую оценку числа $k_{D, \omega}$.

Теперь мы построим области $D_{n}$, апроксимируюшие области $D_{\omega}=F_{\omega}^{-t}(D)$ для всех $\omega \in \Omega_{0}^{*}$ и всех трапеций $D$, определенных вьше. Сначала мы зафиксируем $\omega \in \Omega_{0}^{*}$ и трапецию $D$ и построим две специальные области $D^{\prime}, D^{\prime \prime}$, которые аппроксимируют $D_{\omega}=F_{\omega}^{-t}(D)$ изнутри и извне, т.е. такие, что $D^{\prime} \subset D_{\omega} \subset D^{\prime \prime}$.

Область $D_{\omega}$ получается переносом $D$ по времени назад. Мы рассмотрим ее "траекторию" $D_{s}^{-}=F_{\omega}^{s-t}(D)$ для $0<s<t$, так что $D_{0}^{-}=D_{\omega}$ и $D_{t}^{-}=D$. Мы изучаем форму области $D_{s}^{-}$и то, как она меняется, когда $s$ пробегает интервал от $t$ к 0 . Напомним, что трапеция $D$ соседствует с поршнем в момент времени $t$ (она почти упирается в поршень в это время). Когда $s$ идет от $t$ вниз, область $D_{s}^{-}$отходит от поршня и движется к стенке (как в фильме, прокручиваемом в обратном направлении). В течение 
этого периода отображение $F_{\omega}^{-(t-s)}$, ограниченное на область $D$, линейно и, следовательно, $D_{s}^{-}$остается трапецией. Но так как скорости точек $(x, v) \in D$ изменяются (от $v_{1, \min }$ до $\left.v_{1, \max }\right)$, трапеция будет “скашиваться" - ее "внешняя" сторона $|v|=v_{1, \max }$ будет двигаться в направлении стенки быстрее ее другой стороны $|v|=v_{1, \min }$. К тому времени, когда она достигнет стенки, $D_{s}^{-}$становится длинной косой трапецией, вытянутой на расстояние $O(L)$ вдоль оси $x$. Каждая вертикальная прямая (параллельная оси $v$ ) будет пересекать $D_{s}^{-}$по сегменту длины $O(\Delta t / L)$. Когда $s$ идет дальше вниз, происходит столкновение со стенкой и новая часть области $D_{s}^{-}$возникает прямо через ось $x$, двигаясь теперь в направлении поршня. По форме она будет также длинной узкой трапецией, чья вертикальная “толшина" имеет порядок $O(\Delta t / L)$. По сушеству, она будет такой весь путь (длины $O(L)$ ) от стенки до поршня и соприкасается с поршнем в некоторый момент времени $s_{*}=s_{*}(D)$ (см. рис. 5). Отметим, что до этого времени $D_{s}^{-}$полностью не зависит от $\omega$.

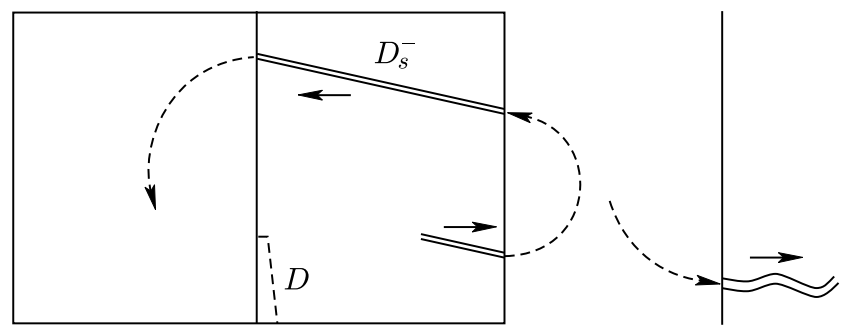

Рис. 5. Область $D_{s}^{-}$. Стрелки показьвают ее движение, когда $s$ убывает. "Выходящая" часть области $D_{s}^{-}$, когда она отходит от поршня, показана справа

После этого столкновения с поршнем появляется новая часть области $D_{s}^{-}$через ось $x$, удаляюшаяся от поршня и двигаюшаяся к стенке. Это есть самая интересная часть, она будет определяться положением и скоростью поршня; следовательно, она будет действительно зависеть от $\omega$. Напомним, что часть области $D_{s}^{-}$, двигающаяся к поршню до столкновения ("входяшая" часть), представляет собой узкую трапецию, ограниченную двумя почти горизонтальньми прямыми с наклоном $O(1 / L)$, находящимися на расстоянии $O(\Delta t / L)$ друг от друга. Мы назовем их верхней и нижней границами области $D_{s}^{-}$. Часть этой области, уходяшая от поршня после столкновения ("выходящая" часть), также имеет верхнюю и нижнюю границы (из обрашения скоростей при столкновении, хотя верхняя граница после столкновения есть образ нижней границы до столкновения, и наоборот), см. рис. 5. Границы выходящей части области $D_{s}^{-}$являются нерегулярными и зависят от $\omega$. Они и нуждаются в аккуратной аппроксимации.

Форма выходяшей гранищы области $D_{s}^{-}$будет определяться скоростью поршня $V(u)$ в интервале $s<u<s_{*}$. Так как столкновение между $D_{s}^{-}$происходит в интервале без столкновений $\left(s_{*}<T_{1}\right)$, мы можем применить результаты $\S 3$. По теореме 3.7 скорость поршня ведет себя как гёльдеровская непрерьвная ${ }^{3}$ функция:

$$
|V(u+h)-V(u)| \leqslant C L^{-1} \ln L \sqrt{h}=: d_{h}
$$

\footnotetext{
${ }^{3}$ Для любого $d>0 d$-плотные множества в пространстве гёльдеровских непрерьвных функций были построены Колмогоровым и Тихомировьм [16]; наши построения здесь аналогичны.
} 
для всех $h \in\left(L^{-2}, 1\right)$. Мы будем рассматривать только такие $h$, для которых $L^{-a_{1}}<$ $h<L^{-a_{2}}$ для некоторых $0<a_{2}<a_{1}<2$. Заметим, что в этом случае

$$
L d_{h} / h \rightarrow \infty \text { и } d_{h} / h \rightarrow 0
$$

при $L \rightarrow \infty$.

Рассмотрим верхнюю границу выходяшей области $D_{s}^{-}$после столкновения. Это непрерьвная кривая, которая может быть параметризована временем столкновения (или временем контакта между $D_{s}^{-}$и поршнем), обозначим его $u$, и тогда она становится параметрической кривой $\left(x_{s}(u), v_{s}(u)\right)$ с параметром $u \in\left(s, s_{*}\right)$. Это означает, в частности , что

$$
F^{w}\left(x_{s}(u), v_{s}(u)\right)=\left(x_{s+w}(u), v_{s+w}(u)\right)
$$

для всех $s<u<s_{*}$ и $w \leqslant u-s$, и точка

$$
F^{u-s}\left(x_{s}(u), v_{s}(u)\right)=\left(x_{u}(u), v_{u}(u)\right)
$$

есть конец верхней границы области $D_{u}^{-}$, т.е. $x_{u}(u)-$ координата поршня в момент времени $u$.

УТВЕРЖДЕНИЕ 1. Для каждого $u \in\left(0, s_{*}-h\right) u$ всех достаточно больиих $L$

$$
\left|x_{u+h}(u+h)-x_{u}(u)\right| \leqslant B \varepsilon_{0} h
$$

$$
\left|v_{u+h}(u+h)-v_{u}(u)\right| \leqslant 3 d_{h} .
$$

ДокАЗАТЕльство. Первая оценка означает, что $|X(u+h)-X(u)| \leqslant B \varepsilon_{0} h$, и следует из (3.44). Чтобы доказать второе неравенство, мы воспользуемся правилом скоростей при столкновении

$$
v_{u}(u)=-v_{u}^{-}(u)+2 V(u)
$$

где $v_{u}^{-}(u)$ есть $v$-координата нижней границы "входящей" части области $D_{u}$, при которой она соприкасается с поршнем (в момент времени $u$ ). Подобным образом,

$$
v_{u+h}(u+h)=-v_{u+h}^{-}(u+h)+2 V(u+h) .
$$

Так как нижняя граница “входящей” части области $D_{s}^{-}-$это прямая линия с наклоном $O(1 / L)$, а скорость поршня ограничена, то

$$
\left|v_{u+h}^{-}(u+h)-v_{u}^{-}(u)\right| \leqslant \text { const } \cdot h / L \leqslant d_{h},
$$

где последнее неравенство следует из (4.34). Из этого и из (4.33) следует (4.36). Утверждение доказано.

Мы фиксируем некоторое $h$, для которого

$$
(\Delta t / L)^{1 / 2} \leqslant h \leqslant 2(\Delta t / L)^{1 / 2} .
$$


Из наших ограничений (4.11) на $\Delta t$ следует, что $L^{-2 / 3} \leqslant h \leqslant L^{-4 / 7}$; значит, (4.34) вьполнено. Заметим также, что

$$
d_{h} /(\Delta t / L) \rightarrow 0 \text { при } L \rightarrow \infty,
$$

следовательно, $d_{h}$ будет значительно меньше “толшины” области $D_{s}^{-}$.

Теперь мы наложим на $(x, v)$-плоскость решетку с шагом $r h$ по $x$ и $r d_{h}$ по $v$, где $r>0$ - достаточно малая постоянная (например, $r=B \varepsilon_{0} / 10$ ). Узлы решетки имеют вид

$$
\left(x_{i}, v_{j}\right)=\left(r h_{i}, r d_{h} j\right), \quad i, j \in \mathbb{Z} .
$$

Сейчас мы готовы определить гранищу области $D^{\prime} \subset D_{0}^{\prime}=F_{\omega}^{-t}(D)$, апроксимируюшей $D_{0}^{-}$изнутри. Мы начнем с верхней гранищы "выходящей" части области $D_{0}^{-}$. Она параметризована как $\left(x_{0}(u), v_{0}(u)\right)$ (см. выше) с $0<u<s_{*}$. Мы рассмотрим дискретные значения параметра $u_{k}=k h, k=0,1, \ldots,\left[s_{*} / h\right]$. Для каждого такого $k$ определим точку

$$
F^{k h}\left(x_{0}(k h), v_{0}(k h)\right)=\left(x_{k h}(k h), v_{k h}(k h)\right) .
$$

Это есть конщевая точка верхней границы области $D_{k h}^{-}$, так что $x_{k h}(k h)=X(k h)$ - координата поршня в момент времени $k h$. Затем мы выберем узел решетки (4.39), ближайший к точке $\left(x_{k h}(k h), v_{k h}(k h)\right)$ и лежаший по ту же сторону от поршня, что и область $D_{k h}^{-}$. Назовем этот узел $\left(x_{k}^{s}, v_{k}^{s}\right)$. Далее мы сдвигаем этот узел вниз (вдоль оси $v$ ) на фиксированное расстояние $10 d_{h}$ и получаем сдвинутую точку $x_{k}^{a}, v_{k}^{a}$ :

$$
x_{k}^{a}=x_{k}^{s}, \quad v_{k}^{a}=v_{k}^{s}-10 d_{h} .
$$

Заметим, что из утверждения 1 (и малости $r$ ) следует

$$
\left|x_{k}^{a}-x_{k+1}^{a}\right|=\left|x_{k}^{s}-x_{k+1}^{s}\right| \leqslant 2 B \varepsilon_{0} h
$$

и

$$
\left|v_{k}^{a}-v_{k+1}^{a}\right|=\left|v_{k}^{s}-v_{k+1}^{s}\right| \leqslant 4 d_{h} .
$$

Пусть

$$
\left(x_{k}^{0}, v_{k}^{0}\right):=F^{-k h}\left(x_{k}^{a}, v_{k}^{a}\right) .
$$

Соединим точки $\left(x_{k}^{0}, v_{k}^{0}\right)$ и $\left(x_{k+1}^{0}, v_{k+1}^{0}\right)$ прямолинейньм отрезком, которьй обозначим $L_{k}$. Затем верхнюю границу области $D^{\prime}$ составим из отрезков $L_{k}$, т.е. положим ее равной

$$
\bigcup_{k=0}^{\left[s_{*} / h\right]-1} L_{k} .
$$

УТВЕРЖДЕНИЕ 2. Верхняя граница области $D^{\prime}$ челиком лежит внутри области $D_{0}^{-}=F_{\omega}^{-t}(D)$. 
ДокАЗАТЕЛЬСтво. Достаточно показать, что каждьй отрезок $L_{k}$ лежит внутри $D_{0}^{-}$. Легко видеть, что ограничение на $L_{k}$ отображения $F^{k h}$ линейно и образ $L_{k}^{a}:=$ $F^{k h}\left(L_{k}\right)$ есть прямолинейный отрезок, соединяюший точки

$$
\left(x_{k}^{a}, v_{k}^{a}\right) \text { и } F^{-h}\left(x_{k+1}^{a}, v_{k+1}^{a}\right)=\left(x_{k+1}^{a}-v_{k+1}^{a} h, v_{k+1}^{a}\right) .
$$

Обозначим через

$$
\left|L_{k}^{a}\right|_{x}=\left|x_{k+1}^{a}-x_{k}^{a}-v_{k+1}^{a} h\right|, \quad\left|L_{k}^{a}\right|_{v}=\left|v_{k+1}^{a}-v_{k}^{a}\right|
$$

длины проекций отрезка $L_{k}^{a}$ на оси $x$ и $v$ соответственно. Заметим, что $v_{1, \min }-B \varepsilon_{0} \leqslant$ $\left|v_{k+1}^{a}\right| \leqslant v_{1, \max }+B \varepsilon_{0}$. Следовательно, из (4.35) вытекает, что

$$
\left(v_{1, \min }-2 B \varepsilon_{0}\right) h \leqslant\left|L_{k}^{a}\right|_{x} \leqslant\left(v_{1, \max }+2 B \varepsilon_{0}\right) h,
$$

т.е. $\left|L_{k}^{a}\right|_{x}$ имеет порядок $h$. Из (4.36) $\left|L_{k}^{a}\right|_{v}<3 d_{h}$. Следовательно, в силу (4.34) наклон отрезка $L_{k}^{a}$ равен $O\left(d_{h} / h\right) \rightarrow 0$. Таким образом, $L_{k}^{a}-$ почти горизонтальный отрезок. Неравенство (4.36) показывает, что верхняя граница $\left(x_{k h}(u), v_{k h}(u)\right)$ области $D_{k h}^{-}$при $k h \leqslant u \leqslant k h+h$, которая лежит прямо над $L_{k}^{a}$, осциллирует в $v$-направлении не более чем на $3 d_{h}$. Сдвиг (4.40) тогда обеспечивает, что отрезок $L_{k}^{a}$ лежит целиком ниже верхней границы области $D_{k h}^{-}$. Из (4.38) следует, что область $D_{k h}^{-}$значительно "толще" расстояния между ее верхней границей и $L_{k}^{a}$; поэтому $L_{k}^{a}$ целиком лежит внутри этой области. Следовательно, $L_{k}=F^{-k h}\left(L_{k}^{a}\right)$ целиком лежит внутри $D_{0}^{-}=F^{-k h}\left(D_{k h}^{-}\right)$; утверждение доказано.

Из уже приведенных аргументов также ясно, что отрезок $L_{k}^{a}$ находится на расстоянии $<20 d_{h}$ ниже действительной верхней границы области $D_{k h}^{-}$, так что площадь между ними ограничена const $\cdot h d_{h}$. Следовательно, общая площадь между верхними гранищами областей $D_{0}^{-}$и $D^{\prime}$ не превосходит

$$
\text { const } \cdot h d_{h} \cdot\left(s_{*} / h\right) \leqslant \text { const } \cdot L d_{h} .
$$

Тем же самьм образом строится нижняя граница новой области $D^{\prime}$. Единственная разница состоит в том, что мы сочетаем отобранные узлы решетки (4.39) с их сдвигами вверх, так что соединяюший их отрезок будет снова находиться внутри $D_{0}^{-}$. Верхняя и нижняя части границы дадут нам новую область, аппроксимируюшую "входяшую" часть области $D_{0}^{-}$после столкновения с поршнем, т.е. часть $F^{-t}\left(D \cap G_{*}(t)\right)$. Отметим, что эта часть может сама испытать одно столкновение со стенкой (в интервале $\left(0, s_{*}\right)$ ); тогда она будет состоять из двух связных компонент, расположенных поперек оси $x$.

Остается аппроксимировать "хорошую" часть области $F_{\omega}^{-}(D)=D_{0}^{-}$, которая не взаимодействует с поршнем, т.е. часть $F_{\omega}^{-t}\left(D \cap G_{0}(t)\right)$. Эта часть, как описывалось вьше, состоит из одной или двух трапеций, ограниченных двумя почти горизонтальными прямыми на расстоянии $O(\Delta t / L)$ друг от друга. Это является легкой задачей. Мы просто заменяем верхнюю (нижнюю) границу области $F^{-t}\left(D \cap G_{0}(t)\right)$ многоугольной линией, соединяюшей некоторые узлы решетки (4.39), выбранные по одному на каждой вертикальной прямой $x=x_{i}$, пересекающей область $D_{s}^{-}$и лежашей внутри (вне) этой области не дальше чем на расстоянии $d_{h}$ от настоящей гранишы (напомним, что $r$ в (4.39) очень мало, так что описанньй выбор возможен). Так строится 
граница области $D^{\prime}$. Отметим, что если мы занумеруем выбранные точки последовательно (скажем, слева направо) и обозначим их $\left(x_{k}^{s}, v_{k}^{s}\right)$, то для соседних узлов будут снова вьполнены соотношения (4.41) и (4.42).

Описанная вьше сложная конструкшия определяет область $D^{\prime} \subset D_{\omega}=F_{\omega}^{-t}(D)$ такую, что

$$
\left|D_{\omega} \backslash D^{\prime}\right| \leqslant \text { const } \cdot d_{h} L=\text { const } \cdot \ln L \sqrt{h} .
$$

Совершенно аналогичньм образом мы построим другую область $D^{\prime \prime}$, которая приближает $D_{\omega}$ извне и для которой вьполнено:

$$
\left|D^{\prime \prime} \backslash D_{\omega}\right| \leqslant \text { const } \cdot d_{h} L=\text { const } \cdot \ln L \sqrt{h}
$$

(мы должны только сдвинуть узлы вдоль верхней границы вверх, а вдоль нижней границы - вниз, так что новые границы будут находиться полностью вне $\left.D_{\omega}\right)$. Так как $|D|=O(\Delta t)$, из неравенств $(4.43),(4.44)$ следует, что

$$
\left|D^{\prime \prime} \backslash D^{\prime}\right| \leqslant \text { const } \cdot \ln L \sqrt{h}(\Delta t)^{-1}|D|,
$$

поэтому мы получаем (4.22) с

$$
\chi^{(3)}=\text { const } \cdot \ln L \sqrt{h}(\Delta t)^{-1} .
$$

Отметим, что $\chi^{(3)} \rightarrow 0$ при $L \rightarrow \infty$ благодаря выбору $h$ в $(4.37)$ и ограничениям на $\Delta t$ B (4.11).

Наши области $D^{\prime}, D^{\prime \prime}$ построены вокруг $D_{\omega}$; следовательно, они зависят от $\omega$. Однако границы областей $D^{\prime}, D^{\prime \prime}$ являются многоугольными линиями, вершины которых выбираются из узлов фиксированной решетки (4.39). Поэтому обшее число различнbl $x$ областей $D^{\prime}, D^{\prime \prime}$ конечно. Мы оценим их число $N_{*}$.

Прежде всего, общее число узлов решетки (4.39) в подходящей области $0<x<L$, $|v|<v_{1, \max }$ равно

$$
K_{1}=\text { const } \cdot(L / h) \cdot\left(2 v_{\max } / d_{h}\right) \leqslant \text { const } \cdot L\left(h d_{h}\right)^{-1} .
$$

Напомним, что область $D_{0}=F^{-t}(D)$ состоит самое большее из четырех связных компонент. Часть $F^{-t}\left(D \cap G_{*}(t)\right)$, или “выходящая" часть области $D_{0}$, возникающая после столкновения с поршнем, состоит не более чем из двух компонент. И часть $F^{-t}\left(D \cap G_{0}(t)\right)$ состоит не более чем из двух компонент, каждая из которых является регулярной трапецией. Верхняя (нижняя) часть границы области $D^{\prime}\left(D^{\prime \prime}\right)$ будет состоять, самое большее, из четырех непересекаюшихся многоугольных линий, построенных на узлах решетки (4.39). В каждой многоугольной линии в качестве первой точки может быть взят любой из $K_{1}$ узлов решетки (см. (4.46)). Но другие точки строятся последовательно, и каждая точка должна быть построена из узла, выбранного из

$$
K_{0}:=\frac{4 B \varepsilon_{0}}{r} \cdot \frac{8}{r}=\mathrm{const}
$$

ближайших соседей предшествуюшего узла, выбранного в соответствии с (4.41), (4.42). Ясно, что каждая многоугольная линия имеет не более

$$
M:=\text { const } \cdot L / h
$$


ребер (вершин). Поэтому общее число способов, какими можно построить одну многоугольную линию, не превосходит $K_{1} K_{0}^{M}$. Общее число способов построить всю границу области $D^{\prime}\left(D^{\prime \prime}\right)$ меньше $K_{1}^{8} K_{0}^{8 M}$. Это определяет границу сверху для числа различных областей $D^{\prime}\left(D^{\prime \prime}\right)$ :

$$
N_{*} \leqslant K_{1}^{8} K_{0}^{8 M} \leqslant \text { const } \cdot L^{8}\left(h d_{h}\right)^{-8} e^{\text {const } \cdot L / h} .
$$

Конечно, экспоненщиальный множитель доминирует здесь, и в него могут быть включены все остальные сомножители.

Мы теперь укажем значение $B_{\Delta t}$ в (4.25)-(4.28):

$$
B_{\Delta t}=c_{7} \sqrt{L / h}
$$

с достаточно малой постоянной $c_{7}>0$. Тогда из (4.27) и (4.48) следует, что

$$
\mathrm{P}\left(\Omega_{0}^{*} \backslash \Omega_{1}^{*}(\Delta t)\right) \leqslant \text { const } \cdot e^{-c_{8} L / h}
$$

с некоторой постоянной $c_{8}>0$. Вспоминая, что для $h$ вьполнено (4.37), получим

$$
\mathrm{P}\left(\Omega_{0}^{*} \backslash \Omega_{1}^{*}(\Delta t)\right) \leqslant \text { const } \cdot e^{- \text {const } \cdot L^{3 / 2}(\Delta t)^{-1 / 2}} .
$$

Так как для $\Delta t$ справедливо (4.11), мы имеем

$$
\mathrm{P}\left(\Omega_{0}^{*} \backslash \Omega_{1}^{*}(\Delta t)\right) \leqslant \text { const } \cdot e^{- \text {const } \cdot L^{11 / 7}} .
$$

В силу (4.49) и (4.37)

$$
B_{\Delta t} \leqslant c_{7} L^{3 / 4}(\Delta t)^{-1 / 4},
$$

откуда следует (4.25), так как $\Delta t \gg L^{-1 / 3}$.

Далее, для каждого $\omega \in \Omega_{1}^{*}(\Delta t)$, каждого $t \in\left(T_{1}, S_{2}-\Delta t\right)$ и любой трапеции $D$, определенной вьше, число частиц $k_{D, \omega}$ в $D$ удовлетворяет $(4.28)-(4.32)$ с $\chi^{(3)}$, заданным неравенством (4.45), и $h$, заданным в (4.37); следовательно,

$$
k_{D, \omega}=\widetilde{\lambda}_{D}+\chi_{D, \omega}
$$

где

$$
\left|\chi_{D, \omega}\right| \leqslant \text { const } \cdot\left(B_{\Delta t} L \sqrt{\Delta t}+L^{2} \ln L \sqrt{h}+L \ln L\right) \leqslant \text { const } \cdot L^{7 / 4} \ln L(\Delta t)^{1 / 4} .
$$

Мы замечаем, что $\widetilde{\lambda}_{D}=O\left(L^{2} \Delta t\right)$, поэтому $\left|\chi_{D, \omega}\right| \ll \widetilde{\lambda}_{D}$ для всех $\Delta t$, удовлетворяюших (4.11). Кстати, из этого легко следует грубая оценка $k_{D, \omega} \leqslant 2 \widetilde{\lambda}_{D} \leqslant$ const $\cdot L^{2} \Delta t$, которая объясняет (4.16) и, следовательно, (4.18).

Использование (4.19) и применение предыдущих оценок к каждому $k_{i}^{ \pm}, i=1,2$, дает

$$
k=L^{2} \int_{D_{1}} \widetilde{p}(x, v, t) d x d v+\chi^{\prime}
$$

где

$$
\left|\chi^{\prime}\right| \leqslant \text { const } \cdot\left[L^{7 / 4} \ln L(\Delta t)^{1 / 4}+L^{2} \delta V \Delta t\right]
$$


Аналогичньм путем мы можем оценить другой случайный фактор в формуле (4.15) главного разложения, именно

и получить

$$
Z=\sum_{j=1}^{k} v_{j}
$$

$$
Z=L^{2} \int_{D_{1}} v \widetilde{p}(x, v, t) d x d v+\chi^{\prime \prime}
$$

с той же самой оценкой сверху на $\chi^{\prime \prime}$, что и на $\chi^{\prime}$.

Использование свойств гладкости функции $\widetilde{p}(x, v, t)$, описанных в тексте, окружающем соотношение $(4.9)$, дает (ср. с (3.38)-(3.40))

$\mathrm{c}$

$$
\begin{aligned}
\int_{D_{1}} \widetilde{p}(x, v, t) d x d v & =\left(\widetilde{Q}_{1}(t)-\widetilde{Q}_{0}(t)\right) \Delta t+\chi^{(4)} \\
\int_{D_{1}} v \widetilde{p}(x, v, t) d x d v & =\left(\widetilde{Q}_{2}(t)-\widetilde{Q}_{1}(t)\right) \Delta t+\chi^{(5)}
\end{aligned}
$$

для $u=4,5$.

$$
\left|\chi^{(u)}\right| \leqslant \text { const } \cdot\left[(\Delta t)^{2} / L+\delta V \Delta t\right]
$$

Таким образом, мы получаем, что

$$
V(t+\Delta t)-V(t)=\widetilde{\mathscr{D}}(t) \Delta t+\chi
$$

где

$$
|\chi| \leqslant \text { const } \cdot\left[L^{-1 / 4} \ln L(\Delta t)^{1 / 4}+\delta V \Delta t+(\Delta t)^{2}\right] .
$$

Напомним, что в силу (4.18) $\delta V=O(\Delta t)$. Теперь легко проверить, что второй и третий члены значительно меньше первого. Тем самьм доказано (4.12)-(4.14). Теорема 4.4 еше не доказана, поскольку мы зафиксировали произвольное значение $\Delta t$, а наше множество $\Omega_{1}^{*}$ зависело от $\Delta t$.

На самом деле, как мы увидим позднее, для нашей главной цели достаточно доказать теорему 4.4 только для одного значения $\Delta t$, а именно для $\Delta t=1 / L^{1 / 7}$. Но требуется не много дополнительных усилий, чтобы доказать нашу теорему для всех $\Delta t$, удовлетворяюших (4.11). Мы сделаем это. Разделим интервал (4.11) на подьнтервалы длины $e^{-L}$, т.е. зафиксируем конечную совокупность точек $(\Delta t)_{n}=n e^{-L}$ для $n=n_{1}, \ldots, n_{2}$ с $n_{1}=L^{-1 / 2}(\ln L)^{2} e^{L}$ и $n_{2}=L^{-1 / 7} e^{L}$. Затем мы определяем

$$
\Omega_{1}^{*}=\bigcap_{n=n_{1}}^{n_{2}} \Omega_{1}^{*}\left((\Delta t)_{n}\right) .
$$

Из (4.51) следует, что

$$
\mathrm{P}\left(\Omega_{0}^{*} \backslash \Omega_{1}^{*}\right) \leqslant \text { const } \cdot e^{L-\text { const } \cdot L^{11 / 7}},
$$

что очевидньм образом достаточно для справедливости оценки (4.10).

Теперь для любого $\Delta t$, удовлетворяющего (4.11), мы найдем такое $(\Delta t)_{n}$, что $\left|\Delta t-(\Delta t)_{n}\right| \leqslant e^{-L}$. Для любого $\omega \in \Omega_{1}^{*} \subset \Omega_{1}^{*}\left((\Delta t)_{n}\right)$ мы имеем все предыдушие оценки с $\Delta t$, замененным на $(\Delta t)_{n}$. Эта замена вносит только экспоненщиально малую погрешность $e^{-L}$ в наши оценки. Это не портит наших оценок, которые все являются полиномиальными по $L$. Теорема 4.4 доказана.

Следуюшая теорема - аналог теоремы 3.5. 
Теорема 4.5. Предположим, что $\varepsilon_{0}>0$ достаточно мало. Для всех достаточно больших $L$, для каждой конфигурации $\omega \in \Omega_{1}^{*}$ и для всех $t \in\left(T_{1}, S_{2}\right)$

(i) существует такая постоянная $B>0$, что

$$
|V(t)|<B \varepsilon_{0}
$$

(ii) существует такая постоянная $C_{0}>0$, что

$$
\left|V(t)-V_{0}(t)\right|<C_{0} L^{-1 / 7} \ln L,
$$

где $V_{0}(t)$ определяется равенством

$$
V_{0}(t)=\frac{\widetilde{Q}_{1}(t)-\sqrt{\widetilde{Q}_{1}^{2}(t)-\widetilde{Q}_{0}(t) \widetilde{Q}_{2}(t)}}{\widetilde{Q}_{0}(t)},
$$

$e c л u \widetilde{Q}_{0}(t) \neq 0, u$

$$
V_{0}(t)=\frac{\widetilde{Q}_{2}(t)}{2 \widetilde{Q}_{1}(t)}
$$

в противном случае.

ДокАЗАТЕльСТВО этой теоремы во многом повторяет доказательство теоремы 3.5 . Первая его половина, вплоть до формулы (3.59), может быть скопирована почти дословно с единственной заменой $S_{1}$ на $S_{2}, Q_{i}$ на $\widetilde{Q}_{i}$ и $\mathscr{D}$ на $\widetilde{\mathscr{D}}$. Мы опускаем эту часть. Остаток доказательства требует более сушественной модификации, и мы проводим его подробно.

Прежде всего, напомним, что в теореме 3.5 мы имели дело с $t \in\left(0, S_{1}\right)$. На интервале $\left(0, S_{1}\right)$ функции $Q_{i}(t), i=0,1,2$, не зависят от $\omega$, они определяются уравнениями (3.9)-(3.12), в которых $p(x, v, t)$ является на самом деле детерминистской плотностью, теперь обозначаемой $\widetilde{p}(x, v, t)$. Следовательно, наши функции $\widetilde{Q}_{i}(t)$ для $t>T_{1}$ являются естественными продолжениями функций $Q_{i}(t)$ за интервал $\left(0, S_{1}\right)=\left(0, T_{1}\right)$, и они обладают теми же самыми свойствами, ср. с (3.13) и (4.9). Поэтому функция $V_{0}(t)$, определяемая равенствами $(4.56),(4.57)$ для $t>T_{1}$, есть продолжение функции $V_{0}(t)$, определяемой равенствами $(3.46)$ и (3.47) на интервале $\left(0, T_{1}\right)$.

Далее, в первой половине доказательства (которую мы опустили, так как она почти совпадает с первой половиной доказательства теоремы 3.5) мы должны ввести $t_{*}<S_{2}$, как первый момент времени, в которьй (4.54) не выполнено. Мы докажем (4.55) для всех $t<t_{*}$ с некоторой постоянной $C_{0}>0$ (не зависящей от выбора $B$ в (4.54), которьй нужно будет еще сделать).

Из оценки (3.45), доказанной для $t \leqslant T_{1}$, следует, что (4.55) вьполнено, по крайней мере, для некоторого $t>T_{1}$. Если (4.55) нарушается для какого-либо $t<t_{*}$, тогда пусть $t \in\left(T_{1}, t_{*}\right)$ будет первьм моментом времени, при котором это нарушение происходит. Обозначим через

$$
\Delta_{0}=L^{-1 / 7}
$$

максимально дозволенное прирашение времени в теореме 4.4. Пусть $s=t-\Delta_{0}$. По теореме 4.4

$$
V(t)=V(s)+\widetilde{\mathscr{D}}(s) \Delta_{0}+\chi,
$$


где

$$
|\chi| \leqslant C L^{-1 / 4} \ln L\left(\Delta_{0}\right)^{1 / 4}=C L^{-2 / 7} \ln L .
$$

В силу аналога соотношения (3.58), полученного в первой половине доказательства этой теоремы,

$$
V_{0}(t)=V_{0}(s)+\chi_{0}
$$

c

$$
\left|\chi_{0}\right| \leqslant \frac{E_{0} \varepsilon_{0} \Delta_{0}}{L}=\frac{E_{0} \varepsilon_{0}}{L^{8 / 7}}
$$

Для краткости положим $U(s)=V(s)-V_{0}(s)$ для всех $s$. Вычитая (4.59) из (4.58), получим

$$
U(t)=U(s)+\widetilde{D}(s) \Delta_{0}+\chi^{\prime}
$$

с $\chi^{\prime}=\chi-\chi_{0}$, так что для больших $L$

$$
\left|\chi^{\prime}\right| \leqslant 2 C L^{-2 / 7} \ln L
$$

Без потери обшности предположим теперь, что $U(t)>0$. Так как в момент времени $t$ неравенство (4.55) не вьполняется, то

$$
U(t) \geqslant C_{0} L^{-1 / 7} \ln L
$$

Рассмотрим два случая. Если $U(s) \leqslant 0$, тогда по аналогу соотношения (3.59)

$$
U(t) \leqslant|\widetilde{\mathscr{D}}(s)| \Delta_{0}+\left|\chi^{\prime}\right| \leqslant E_{2}|U(s)| \Delta_{0}+\left|\chi^{\prime}\right| \ll L^{-1 / 7} \ln L
$$

для больших $L$, что противоречит (4.62). Если $U(s)>0$, тогда снова в силу $(4.60)$ и аналога соотношения (3.59)

$$
U(t)<U(s)\left[1-E_{1} \Delta_{0}\right]+\chi^{\prime}
$$

поэтому

$$
U(s)>\frac{U(t)-\chi^{\prime}}{1-E_{1} \Delta_{0}}>\left(U(t)-\chi^{\prime}\right)\left(1+E_{1} \Delta_{0}\right)>U(t)+U(t) E_{1} \Delta_{0}-2 \chi^{\prime} .
$$

Теперь если $C_{0}$ в (4.55) достаточно большое, скажем, $C_{0}=3 C / E_{1}$, то $U(t) E_{1} \Delta_{0}>2 \chi^{\prime}$ в силу (4.62) и (4.61). Из этого факта и из (4.63) вытекает, что $U(s)>U(t)$, так что (4.55) нарушается в более ранний момент времени $s<t$, что противоречит предположению о $t$. Итак, (4.55) доказано для всех $t<t_{*}$ и $C_{0}=3 C / E_{1}$.

Оставшаяся часть доказательства теоремы 3.5 может быть дословно повторена, что и завершает доказательство теоремы 4.5 .

Наконец, мы доказьваем сходимость случайной траектории поршня при $L \rightarrow \infty$ к решению $Y(t / L), W(t / L)$ гидродинамических уравнений из $\S 2$ для всех $t \in\left(T_{1}, S_{2}\right)$. 
ТеОрема 4.6. Предположим, что $\varepsilon_{0}>0$ в (P5) достаточно мало. Тогда для всех больших $L$ и всех $\omega \in \Omega_{1}^{*}$ существует такая постоянная $C>0$, что

$$
\left|Y_{L}(\tau, \omega)-Y(\tau)\right| \leqslant \frac{C \ln L}{L^{1 / 7}}
$$

$u$

$$
\left|W_{L}(\tau, \omega)-W(\tau)\right| \leqslant \frac{C \ln L}{L^{1 / 7}}
$$

для всех $\min \left\{\tau_{1}, T_{1} / L\right\}<\tau<\min \left\{\tau_{2}, S_{2} / L\right\}$. Кроме того,

$$
T_{2}-S_{2} \leqslant C \varepsilon_{0} L
$$

$u$

$$
\tau_{2}-S_{2} / L \leqslant C \varepsilon_{0}
$$

Флуктуачии функиии $S_{2}=S_{2}(\omega)$ для $\omega \in \Omega_{1}^{*}$ ограничены:

$$
\sup _{\omega, \omega^{\prime} \in \Omega_{1}^{*}}\left|S_{2}(\omega)-S_{2}\left(\omega^{\prime}\right)\right| \leqslant \frac{C \ln L}{L^{1 / 7}} .
$$

ДокАЗАТЕльство. Согласно (2.29) детерминистская функция $Y(\tau)$ удовлетворяет уравнению

$$
d Y(\tau) / d \tau=F(Y, \tau), \quad Y(0)=1 / 2 .
$$

Из теорем 3.7 и 4.5 следует, что для всех $\omega \in \Omega_{1}^{*}$ случайная траектория удовлетворяет уравнению

$$
\partial Y_{L}(\tau, \omega) / \partial \tau=F(Y, \tau)+\chi(\tau, \omega), \quad Y_{L}(0, \omega)=1 / 2
$$

с некоторым

$$
|\chi(\tau, \omega)|<\frac{C \ln L}{L^{1 / 7}} .
$$

Напомним, что $|\partial F(Y, \tau) / \partial Y| \leqslant \kappa($ см. (2.30)). Следовательно, для разности

$$
Z_{L}(\tau, \omega):=Y_{L}(\tau, \omega)-Y(\tau)
$$

вьполнено:

$$
\left|Z_{L}^{\prime}(\tau, \omega)\right| \leqslant \kappa\left|Z_{L}(\tau, \omega)\right|+\frac{C \ln L}{L^{1 / 7}}
$$

и $Z_{L}(0, \omega)=0$. Применение стандартного неравенства Гронуолла из теории дифференциальных уравнений (см., например, лемму 2.1 в [26]) дает неравенства

$$
\left|Z_{L}(\tau, \omega)\right| \leqslant \frac{C \ln L}{\kappa L^{1 / 7}}\left(e^{\kappa \tau}-1\right)
$$

и

$$
\left|Z_{L}^{\prime}(\tau, \omega)\right| \leqslant \frac{C \ln L}{L^{1 / 7}} e^{\kappa \tau}
$$

для всех $\tau<S_{2} / L$, из которых следуют (4.64) и (4.65). 
Неравенство (4.65) дает нам возможность применить предложение $4.1 \mathrm{c} \Delta=C L^{-1 / 7}$ и тем самым доказать (4.66). Теперь мы используем те же аргументы, что и в доказательстве теоремы 3.8. Согласно (4.65) случайные флуктуации скорости поршня ограничены величиной $C L^{-1 / 7} \ln L$. Следовательно, случайные флуктуации скоростей частиц, испытавших одно или два столкновения с поршнем, ограниченывеличиной $4 C L^{-1 / 7} \ln L$. Случайные флуктуации и поршня, и частиц в каждьй момент времени $t<\min \left\{\tau L, S_{2}\right\}$ ограничены теми же самыми величинами (возможно, с отличным значением $C$ ) по координате $y=x / L$. Из этого следует (4.68) тем же самьм путем, как (3.92) в теореме 3.8. Теперь мы можем применить предложение 4.1 к детерминистской динамике, определенной в $\S 2$, чтобы получить тот же результат и, следовательно, доказать (4.67). Теорема 4.6 доказана.

Как показывает (4.68), предел

$$
\tau_{* *}:=\lim _{L \rightarrow \infty} S_{2}(\omega) / L
$$

не зависит от $\omega \in \Omega_{1}^{*}$. Из (4.67) и оценок в лемме 2.9 мы имеем $\left|\tau_{* *}-2 / v_{\max }\right| \leqslant$ const $\cdot \varepsilon_{0}$.

Сходимость, утверждаемая теоремой 1.2 , теперь доказана на интервале $\left(0, \tau_{* *}\right)$, но, в обшем случае, $\tau_{* *}<\tau_{*}$ с $\tau_{*}-\tau_{* *}=O\left(\varepsilon_{0}\right)$, так что мы можем все еще быть на $O\left(\varepsilon_{0}\right)$ удалены от нашего желаемого значения $\tau_{*}$. Для того чтобы распространить наши результаты на весь путь до $\tau_{*}$, нам нужно переопределить $S_{2}$ и окрестность $\mathscr{X}_{1}$, введенную в (4.4), более аккуратно. Мы должны положить

$$
\begin{aligned}
\mathscr{X}_{1}(t)= & \left\{(x, v) \in G^{+}(t): x=X(t)+0, v<0\right\} \\
& \cup\left\{(x, v) \in G^{+}(t): x=X(t)-0, v>0\right\} .
\end{aligned}
$$

Область $G^{+}(t)=F^{t}\left(G^{+}\right)$случайна (она зависит от $\omega$ ), т.е. мы должны были бы писать $G^{+}(t)=G^{+}(t, \omega)$; именно поэтому мы не смогли принять определение $\mathscr{X}_{1}(t)$, данное ранее, и выбрали более грубое определение (4.4). Но теперь, как мы только что показали в доказательстве теоремы 4.6, случайные флуктуации скоростей и положений (по координате $y$ ) частиц и поршня ограничены const $\cdot L^{-1 / 7} \ln L$. Поэтому в каждый момент времени $t<\min \left\{\tau_{2} L, S_{2}\right\}$ все области $G^{+}(\tau, \omega)$ близки друг к другу - расстояние между $G^{+}(\tau, \omega)$ и $G^{+}\left(\tau, \omega^{\prime}\right)$ для $\omega, \omega^{\prime} \in \Omega_{1}^{*}$ в метрике Хаусдорфа на координатной плоскости $y, v$ ограничено const $\cdot L^{-1 / 7} \ln L$. По той же причине каждая область $G^{+}(\tau, \omega)$ будет $O\left(L^{-1 / 7} \ln L\right)$-близка по метрике Хаусдорфа к детерминистской области $\mathscr{G}^{+}(t / L)$, определенной в $\S 2$. Следовательно, мы можем легко сделать $G^{+}(t)$ в (4.71) независимой от $\omega$, скажем, взяв объединение

$$
G^{+}(t)=\bigcup_{\omega \in \Omega_{1}^{*}} G^{+}(t, \omega) .
$$

Важно отметить, что все частицы газа, сталкиваюшиеся с поршнем в момент времени $t$, будут для каждого $\omega \in \Omega_{1}^{*}$ принадлежать $\mathscr{X}_{1}$, определенному равенствами $(4.71),(4.72)$. С новым определением множества $\mathscr{X}_{1}$, заменившим $(4.4)$, и с $S_{2}$, измененным соответственно, мы получаем, что

$$
\lim _{L \rightarrow \infty}\left|S_{2} / L-\tau_{*}\right|=0
$$


что следует из $O\left(L^{-1 / 7} \ln L\right)$-близости случайной динамики к детерминистской динамике на $(y, v)$-плоскости. Тем самьм мы распространили все наши результаты на интервал $\left(0, \tau_{*}\right)$.

\section{$\S 5$. После второго перестолкновения}

Главная цель нашего анализа в $\S 3$ и $\S 4$ состояла в том, чтобы доказать, что при подходяших начальных условиях случайные флуктуации движения массивного поршня в замкнутом сосуде, заполненном идеальным газом, малы и исчезают в термодинамическом пределе. Однако мы можем проконтролировать эти флуктуации эффективно только до тех пор, пока окружаюший газ частиц описывается пуассоновским процессом, т.е. в интервале $0<\tau<\tau_{1}$ без перестолкновений. В этом случае случайные флуктуации ограничены константой const $\cdot L^{-1} \ln L$ (см. теорему 3.8 ). С точностью до логарифмического множителя эта оценка оптимальна согласно теореме 1.1, принадлежащей Холли.

В интервале с одним перестолкновением $\tau_{1}<\tau<\tau_{*}$ ситуация иная. Распределение вероятностей частиц газа, испытавших одно столкновение с поршнем, перестает быть пуассоновским, в нем имеются внутренние коррелящии. Мы можем только показать, что случайные флуктуации остаются ограниченными величиной $L^{-1 / 7}$ (см. теорему 4.6). Возможно, наша оценка далека от оптимальной, но численные эксперименты, о которых идет речь ниже, показьвают, что случайные флуктуации на самом деле растут внутри интервала с одним перестолкновением и после него.

В настоящее время мы не знаем, могут ли быть наши методы или результаты распространены за критическое время $\tau_{*}$, этот вопрос остается открытьм. Мы подчеркиваем, что наша теорема 4.6 представляет собой первьй строгий результат, относяшийся к эволющии поршня в идеальном газе, когда большинство или все его частищы столкнулись с поршнем более одного раза.

Для того чтобы понять, что происходит за критическим временем $\tau_{*}$ и, в частности, будут ли расти или оставаться малыми случайные флуктуации, мы провели эксперименты и эвристическое изучение динамики поршня в большом временном масштабе. Далее мы описываем наши наблюдения и обсуждаем дальнейшие исследования в этом направлении. Подробньй отчет о нашей работе можно найти в [4].

Мы полагаем начальную плотность равной

$$
\pi_{0}(y, v)=\pi_{0}(|v|)= \begin{cases}1 & \text { при } 0.5 \leqslant|v| \leqslant 1 \\ 0 & \text { в других случаях. }\end{cases}
$$

Она удовлетворяет нашим требованиям $(\mathrm{P} 1)-(\mathrm{P} 5)$, в частности, $v_{\min }=0.5, v_{\max }=1$ и, что наиболее важно, $\varepsilon_{0}=0$. Поэтому, в силу следствия 2.13 решение гидродинамических уравнений тривиально: $Y(\tau) \equiv 0.5, W(\tau) \equiv 0$ и $\pi(y, v, \tau) \equiv \pi_{0}(y, v)$ для всех $\tau>0$.

Для задания начальной конфигурации мы использовали датчик случайных чисел, описанный в [23]. Для нашей плотности (5.1) координаты $x$ и $v$ всех частиц являются независимыми случайньми величинами, равномерно распределенными в их диапазонах: $0<x<L$ и $v_{\min }<|v|<v_{\max }$. Наша компьютерная программа сначала 
выбирает число частиц $N$ согласно распределению Пуассона со средним $L^{3}$, а затем генерирует все $\left(x_{i}, v_{i}\right), 1 \leqslant i \leqslant N$, независимо в соответствии с их равномерными распределениями. Параметр $L$ изменялся в наших экспериментах от $L=30$ до $L=300$. Для $L=300$ система содержит $\approx L^{3}=27000000$ частиц.

После генерации случайных начальных данных программа рассчитывает динамику, используя законы упругого столкновения (1.2), (1.3). Все вычисления были выполнены с двойной точностью для координат и скоростей всех частищ, выгисленных индивидуально.

На рис. 6 представлена типичная траектория поршня. Здесь $L=100$. Положение и время задано в гидродинамических переменных $Y=X / L, 0<Y<1$, и $\tau=t / L$.

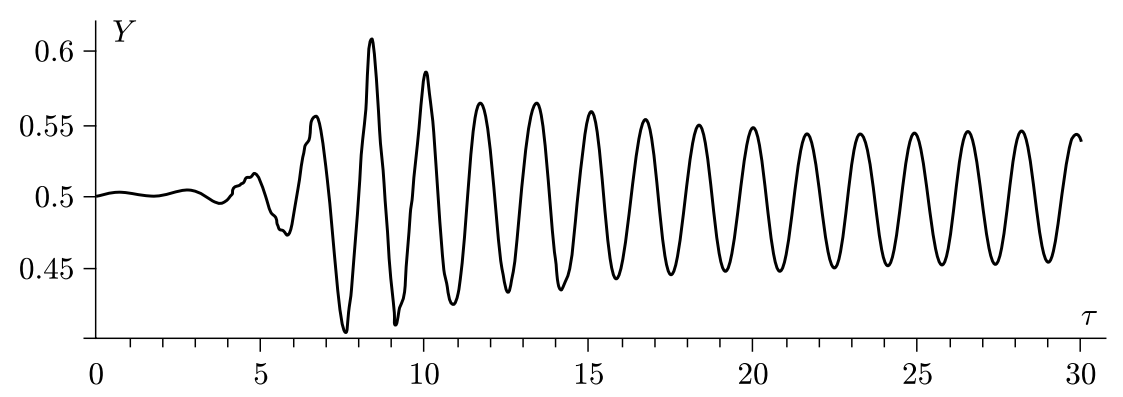

Рис. 6. Координата поршня $Y$ как функция времени $\tau$. Здесь $L=100, N=1000229$

Сначала поршень едва заметно двигается около своей стационарной точки $y=0.5$. Затем в интервале между 3 и 5 случайные вибрации поршня растут и становятся вполне видимыми в $y$-масштабе, но еше некоторое (недолгое) время они вьглядят случайными подобно траектории броуновского движения. После этого поршень начинает двигаться взад и вперед вдоль оси $y$ более регулярным образом, отклоняясь все дальше от стационарной точки $y=0.5$. Очень скоро, при $\tau=\tau_{\max } \approx 8$, качание поршня достигает своего максимума, $(\Delta Y)_{\max }=\max \left|Y_{L}(\tau)-0.5\right| \approx 0.1$. Затем осциллящии поршня затухают по размеру и, кажется, стабилизируются с амплитудой $A \approx 0.04$. Одновременно траектория поршня сглаживается и становится периодической с периодом $\tau_{\text {per }} \approx 1.63$. Скорость поршня $W(\tau)$ ведет себя аналогичньм образом, см. рис. 7 .

И координата, и скорость поршня продолжают совершать почти совершенные гармонические колебания в течение долгого времени с тем же периодом $\tau_{p e r} \approx 1.63$ (он не зависит от $L)$, но амплитуды и $Y(\tau)$ и $W(\tau)$ медленно убывают, см. рис. 8.

Колебания поршня с убываюшей амплитудой могут быть приближенно описаны в интервале $20<\tau<1000$ формулой:

$$
Y(\tau) \approx A e^{-\lambda(\tau-20)} \sin \omega(\tau-\alpha)
$$

с $A=0.046$ и некоторой постоянной $\lambda>0$. Соответственно $W=d Y / d \tau$ в том же интервале $20<\tau<1000$ имеет вид:

$$
\begin{aligned}
W(\tau) \approx & -\lambda Y(\tau)+A e^{-\lambda(\tau-20)} \omega \cos \omega(\tau-\alpha) \\
= & A e^{-\lambda(\tau-20)}[-\lambda \sin \omega(\tau-\alpha)+\omega \cos \omega(\tau-\alpha)] \\
= & A_{1} e^{-\lambda(\tau-20)} \sin \omega(\tau-\beta)
\end{aligned}
$$

с $A_{1}=A \sqrt{\omega^{2}+\lambda^{2}}$ и некоторым $\beta$, связанным с $\alpha$. 


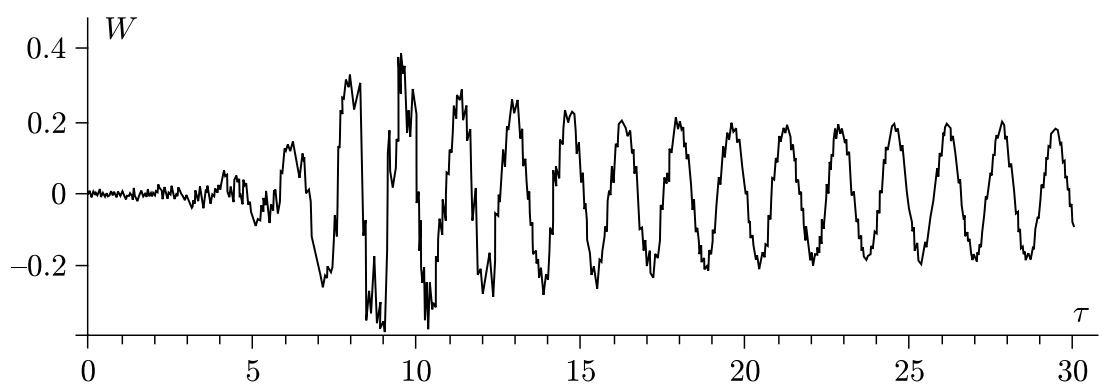

Рис. 7. Скорость поршня $W$ как функция времени $\tau$. Тот же эксперимент, что на рис. 6

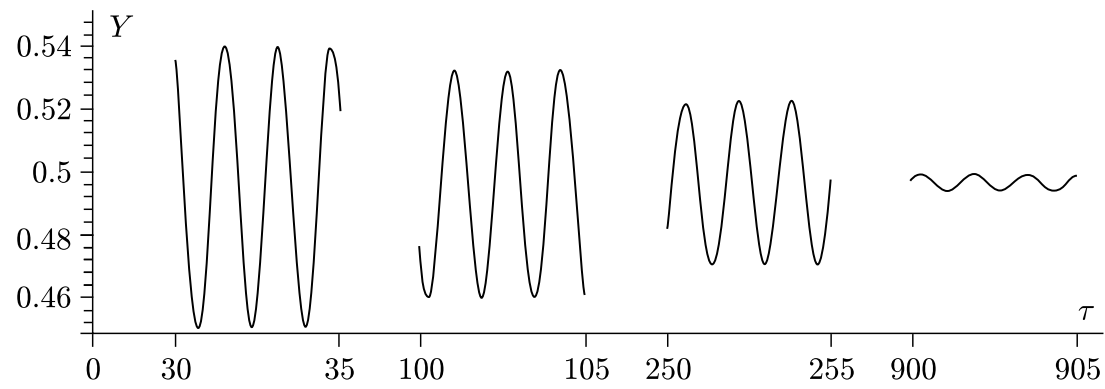

Рис. 8. Координата $Y$ поршня в интервалах $(30,35),(100,105),(250,255)$ и $(900,905)$.

Тот же эксперимент, что на рис. 6 и 7

Чтобы проверить, сколь хорошо наше предсказание (5.2) согласуется с экспериментальными данными, мы вычислили амплитуду $A(\tau)$ как функцию времени $\tau$, подставляя вместо $Y(\tau)$ функцию $A \sin \omega(\tau-\alpha)$ “локально" на интервале $(\tau-5, \tau+5)$ для каждого $\tau$. Рис. 9 показывает график $A(\tau)$ в логарифмической шкале, который выглядит почти линейным на интервале $30<\tau<800$.

Мы использовали метод наименьших квадратов для того, чтобы получить $\lambda=$ 0.00264 для графиков, показанных на рис. 6-9. Так как $\lambda$ мало, колебания в действительности умирают очень медленно. Время "полураспада" (время, за которое амплитуда уменьшается вдвое) равно $\tau_{1 / 2}=\lambda^{-1} \ln 2 \approx 263$. Параметр $\lambda$ и, следовательно, $\tau_{1 / 2}$ зависят от размера $L$ системы. Мы оценили численно, что $\tau_{1 / 2} \sim L^{1.3}$ и поэтому $\lambda \sim L^{-1.3}$.

Ключевые характеристики траектории поршня, описанной выше, в частности, $(\Delta Y)_{\max }, W_{\max }, A, \tau_{\text {per }}$ вьглядят как не зависящие от $L$. Даже для $L=300$ (наибольшая система, проверенная экспериментально) поршень испытьвает большие колебания, очень похожие на те, что показаны на рис. 6 и 7 . Некоторые другие величины, такие, как время $\tau_{1 / 2}$ и связанное с ним $\lambda$, зависят определенно от $L$.

Но самое важное, что время наибольших осцилляций $\tau_{\max }$ и связанное с этим время появления неустойчивого поведения $\tau_{c}$ (см. ниже), похоже, медленно растет вместе с $L$, этот рост очень близок к $\log L$. Для того чтобы понять этот факт, мы посмотрели на механизм возникновения и развития случайных флуктуаций положения и скорости поршня, изображенных на рис. 6 и 7. С этой целью мы нарисовали гистограмму (эмпирической) плотности частиц газа в $(y, v)$-плоскости в различные мо- 


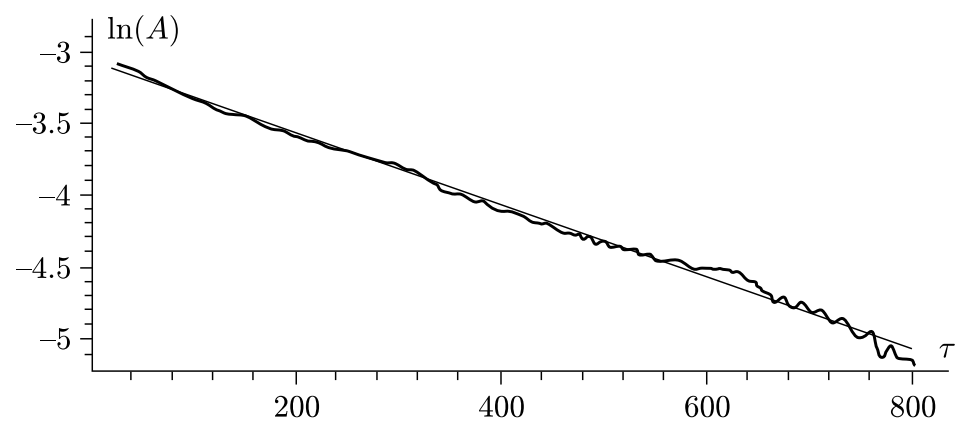

Рис. 9. Амплитуда $A(\tau)$ в логарифмической шкале: экспериментальная кривая (жирная) и подходящая прямая (тонкая). Тот же эксперимент, что на рис. 6,7 и 8

менты времени $0<\tau<30$ (см. рис. 10). Начальная плотность (в момент времени 0 ) почти равномерна в области $0<x<L$ и $v_{\min }<|v|<v_{\max }$ (отклонения в начальной конфигурации существуют всегда, так как она порождена случайно). Далее, в интервале $0<\tau<1$ поршень испытьвает случайные столкновения с частицами и приобретает скорость порядка $M^{-1 / 2}=O(1 / L)$ (см. теорему 1.1). Эти малые флуктуации скорости поршня приводят к большим изменениям скоростей частиц, которые покидают поршень после столкновения согласно правилу (1.3). В частности, частищы, уходящие от поршня справа от него, обладают скоростями в интервале $\left(v_{\min }+2 W(\tau), v_{\max }+2 W(\tau)\right)$, в то время как такие же частицы слева от поршня имеют скорости в интервале $\left(-v_{\min }+2 W(\tau),-v_{\max }+2 W(\tau)\right)$. Следовательно, область в $(y, v)$-плоскости, в которой плотность частиц положительна, больше уже не является прямоугольником с прямьми сторонами, теперь ее границы - это кривые, форма которых почти повторяет график случайной скорости поршня $W(\tau)$. Хотя вариации порядка $O(1 / L)$ этих граничных кривых могут оказаться малыми, решающее значение имеет то, что по разные стороны от поршня они движутся в противоположных направлениях. В самом деле, когда $W(\tau)>0$, тогда уходяшие частицы по правую сторону от поршня ускоряются, а уходяшие частицы по левую сторону замедляются. Когда $W(\tau)<0$, возникает противоположная ситуация.

Далее, частицы, уже столкнувшиеся с поршнем, двигаются к стенке и возврашаются назад к поршню. Теперь их плотность менее регулярна, чем вначале, - области $(x, v)$-плоскости, где эта плотность положительна, становятся криволинейными. Когда частицы ударяются о поршень, они заставляют его колебаться взад и вперед сильнее, чем прежде, так как скорости входящих частиц с противоположных сторон поршня теперь отрицательно коррелированы. Когда частицы с правой стороны убыстряются, частицы слева замедляются, и наоборот. Флуктуации плотностей газа по разные стороны от поршня "конфигурируются" так, чтобы толкать поршень сильнее, с “удвоенной” силой. Благодаря этому происходит явление резонансного типа, дестабилизируюшее движение поршня, и флуктуации скорости поршня $W(\tau)$ становятся больше, чем прежде. Скорости вновь уходящих от поршня частиц будут снова расти и падать в противоположных направлениях в большем масштабе, чем прежде.

$\mathrm{C}$ течением времени описанное явление будет повторяться снова и снова со все большими и большими флуктуациями скоростей частиц газа и поршня до тех пор, пока рас- 


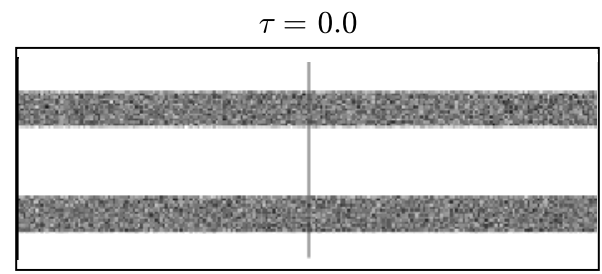

$\tau=4.2$

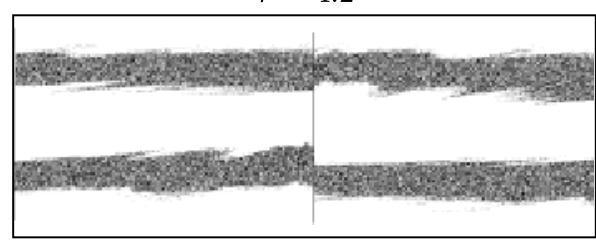

$\tau=7.4$

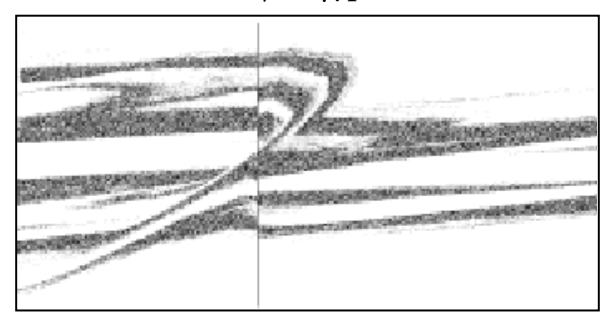

Рис. 10. Шесть снимков эмпирической плотности газа (в $(x, v)$-плоскости) в моменты времени $\tau=0,2.3,4.2,5.9,7.4$ и 18.6

пределение частиц газа не разушится полностью. Для $L=100$ и $t \approx 10$ образуются два больших кластера частиц, по одному с каждой стороны от поршня. Когда один кластер будет "бомбардировать" поршень, другой будет двигаться прочь от него и ударяться об стенку, затем они поменяются ролями. Размеры кластеров в $y$-направлении около $0.3-0.5$, а скорости частиц меняются от 0.2 до 1 . Средняя скорость $\approx 0.5-0.6$ и, таким образом, кластеры "стучат" по поршню с периодом 1.6-2.0, которьй близок к экспериментально определенному периоду колебаний поршня (см. вьше).

На рис. 10 показано шесть мгновенных снимков эмпирической плотности частиц газа в разные моменты времени. При $\tau=0$ газ заполняет (почти равномерно) два прямоугольника $\{(y, v): 0.5<|v|<1,0<y<1\}$. При $\tau=2.3$ можно заметить некоторую рябь на границах этих прямоугольников. При $\tau=4.2$ иррегулярность границ возрастает и при $\tau=5.9$ прямоугольная форма областей разрушается. Образуется два больших кластера частиц, оба в верхней полуплоскости $v>0$, т.е. в это время кластеры движутся вправо (один - в сторону поршня, другой - от него). Позднее носитель плотности принимает странные очертания $(\tau=7.4)$, но, по существу, сглаживается и медленно переходит к сходимости к максвелловскому распределению $(\tau=18.6)$, описанному ниже.

Проведенный анализ показывает, что флуктуации скорости поршня в грубом приближении возрастают в постоянное число раз за каждьй интервал времени длины единица. Действительно, начальная случайная флуктуация $W_{a} \sim O(1 / L)$ приводит к дополнительным изменениям скоростей уходяших частиц на $2 W_{a}$. Когда эти части- 
цы возврашаются назад к поршню (через время $\Delta \tau \approx 1$ ), его скорость подскакивает до уровня $2 W_{a}$. Затем вновь уходяшие частицы приобретают дополнительную скорость $4 W_{a}$ и т. д. За каждый единичный интервал времени размер фллуктуаций удваивается. Это очевидное сверхупрощенное описание реальной динамики тем не менее приводит к разумной гипотезе

$$
W_{a}(\tau) \approx \frac{C R^{\tau}}{L}
$$

где $W_{a}(\tau)$ - типичная фллукуация скорости поршня в момент времени $\tau$, а $C, R>0$ постоянные.

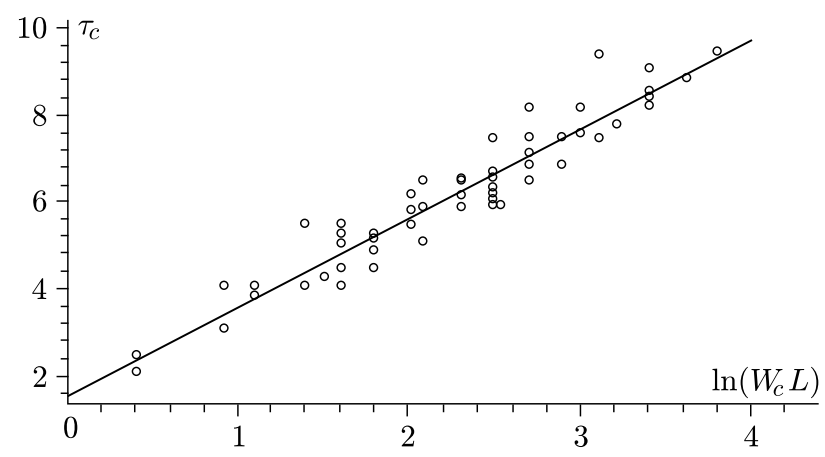

Рис. 11. Значение $\tau_{c}$ как функции от $\ln \left(W_{c} L\right)$ : экспериментальная кривая и подходящая прямая

Мыпроверяли предыдущую формулу численно следующим образом. Пусть $W_{c}>0$ - некоторое выбранное критическое значение скорости поршня и $\tau_{c}=\inf \{\tau>0$ : $\left.|W(\tau)|>W_{c}\right\}-$ (случайное) время, когда $W_{c}$ впервые превьшено. Это время играет роль "начала" больших фллукуаций скорости поршня. На основании (5.4) можно ожидать, что

$$
\tau_{c} \approx \frac{\ln \left(W_{c} L / C\right)}{\ln R}
$$

т.е. $\tau_{c}$ растет, как $\ln L$, когда $L$ возрастает.

Мы нашли экспериментально $\tau_{c}$ для $W_{c}=0.1$ и $W_{c}=0.15$ и проверили, что $(5.5)$ хорошо согласуется с полученньми значениями, см. рис. 11. По методу наименьших квадратов мы нашли $C=0.45$ и $R=1.6$.

Суммируя наши экспериментальные наблюдения, мы приходим к выводу о том, что случайные фолуктуации плотности $p_{L}(y, v, \tau)$ и координаты поршня $Y_{L}(\tau)$ растут экспоненциально со временем $\tau$ и в моменты времени $\tau \sim \log L$ они становятся большими даже в макроскопическом масштабе. В этой точке эволюция системы далеко отклоняется от решения гидродинамических уравнений (H1)-(H4), и поведение системы впоследствии не описьвается этими уравнениями.

Интересно, что наши наблюдения не указывают на то, что утверждаемая теоремой 1.2 сходимость (1.12), (1.13) не имеет места на каком-либо интервале времени. В самом деле, если случайные флуктуации ведут себя как $C R^{\tau} / L$ (как предсказано в (5.4)), тогда $(1.12),(1.13)$ должны были бы вьполняться при $L \rightarrow \infty$ на каждом 
конечном интервале $\left(0, \tau_{*}\right)$. Однако строгое доказательство этого факта остается интригующей проблемой.

Далее, мы проверили численно и эвристически, каково асимптотическое поведение системы при $\tau \rightarrow \infty$. Из физических соображений [1] мы надеемся, что система приближается к тепловому равновесию (см. §1), т.е. распределение скоростей частиц газа должно сходиться к максвелловскому.

Мы использовали статистический критерий Колмогорова-Смирнова для проверки сходимости распределения скорости к нормальному закону. Для любого заданного времени $\tau>0$ пусть

$$
F_{\tau}(u)=\#\left\{i: v_{i}<u\right\} / N
$$

- эмпирическая (кумулятивная) функция распределения скоростей частиц. Для соответствующей нормальной функции распределения $\Phi(x)$ мы вычисляем

$$
D_{\tau}=\sup _{-\infty<u<\infty}\left|F_{\tau}(u)-\Phi(u)\right|
$$

Сначала $D_{0} \approx 0.245$ для нашего выбора $\pi_{0}(v)$ в (5.1). Если бы скорости $v_{i}$ были независимыми нормальными случайными величинами, тогда $D_{\tau}$ было бы порядка $O(1 / \sqrt{N})$, а произведение $D_{\tau} \sqrt{N}$ имело бы определенное предельное распределение (см. например, [22]). В частности, известно, что вероятность $\mathrm{P}\left(D_{\tau} \sqrt{N}>1\right) \approx 0.2$. Основьваясь на этом, мы выбрали в качестве определения времени сходимости к равновесию величину

$$
\tau_{\text {eq }}=\inf \left\{\tau>0: D_{\tau} \sqrt{N}<1\right\}
$$

Мы оценили $\tau_{\text {eq }}$ для различных $L$ и нашли, что $\tau_{\text {еq }} \approx a L^{b}$ с некоторыми постоянными $a, b>0$. С помощью метода наименшших квадратов, примененного к экспериментальным точкам, мы нашли, что $a=0.18$ и $b=2.47$.

График произведения $S=D_{\tau} \sqrt{N}$ как функции от $\tau$ дан на рис. 12 (для одного из вариантов с $L=40)$. Он показывает, что после начального резкого падения на участке $0<\tau<20$ статистика $S$ убывает экспоненщиально по $\tau$. Другая часто используемая (и популярная среди экспериментаторов) статистика для меры близости к нормальному распределению определяется как

$$
S^{\prime}=3-\frac{M_{4}}{M_{2}^{2}}
$$

где $M_{2}$ и $M_{4}$ - второй и четвертый моменты эмпирического распределения скорости соответственно. Рис. 12 показывает, что $S^{\prime}$ сходится, подобно $S$, к нулю (тот же вариант с $L=40)$.

Сходимость к тепловому равновесию может быть также объяснена из математических соображений. Если фиксировать три интеграла движения - общую энергию $E$ и число частиц $N_{L}$ и $N_{R}$ слева и справа от поршня - то динамика этой системы, как мы сейчас покажем, может быть стандартными методами сведена к бильярдной системе в многомерном полиэдре.

Пусть $\left\{x_{i}\right\}, i=1, \ldots, N_{R}$, обозначает произвольно упорядоченные $x$-координаты частиц справа от поршня, а $\left\{x_{i}\right\}, i=-1, \ldots,-N_{L},-$ слева от него. Положим 


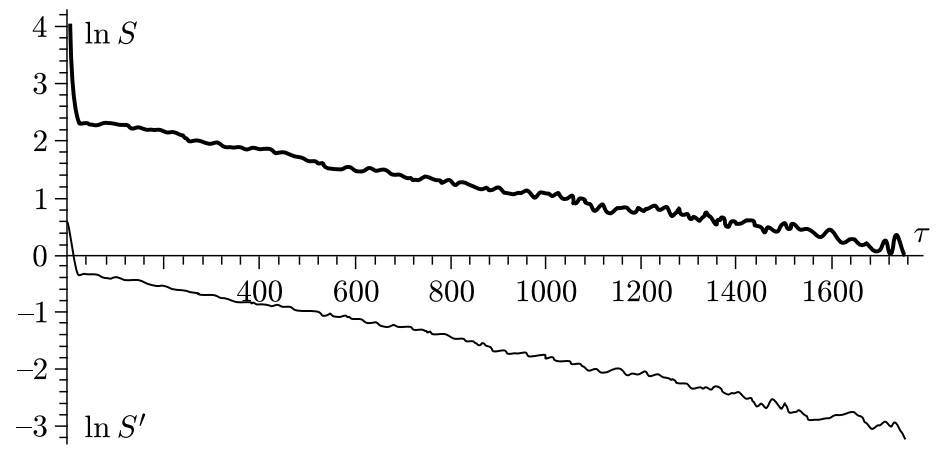

Рис. 12. $\ln S$ (толстая линия) и $\ln S^{\prime}$ (тонкая линия) как функции от $\tau$

$x_{0}=X \sqrt{M}$, где $X$ - координата поршня, а $M$ - его масса. Тогда конфигурационное пространство системы (в координатах $x_{i},-N_{L} \leqslant i \leqslant N_{R}$ ) является полиэдром $Q \subset \mathbb{R}^{N+1}$ (напомним, что $N=N_{L}+N_{R}$ ), определяемьм неравенствами:

$$
0 \leqslant x_{-N_{L}}, \ldots, x_{-1} \leqslant x_{0} / \sqrt{M} \leqslant x_{1}, \ldots, x_{N_{R}} \leqslant L .
$$

Известно, что динамика нашей механической системы соответствует динамике бильярда в $Q$ (см. [6]). Это означает, что точка $\mathbf{q} \in Q$ конфигурационного пространства движется свободно, испытывая упругие столкновения на границе $\partial Q$. Вектор скорости

$$
\mathbf{p}=\dot{\mathbf{q}}=\left\{v_{-N_{L}}, \ldots, v_{-1}, V \sqrt{M}, v_{1}, \ldots, v_{N_{R}}\right\}
$$

имеет постоянную длину, так как $\|\mathbf{p}\|^{2}=2 E=$ const. Следовательно, фазовое пространство этой бильярдной системы есть $\mathscr{M}=Q \times S_{\rho}^{N}$, где $S_{\rho}^{N}-N$-мерная сфера pадиуса $\rho=\sqrt{2 E}$.

Бильярдная система имеет лиувиллеву инвариантную меру $\mu$ на $\mathscr{M}$, являюшуюся произведением равномерной меры на полиэдре $Q$ и равномерной (лебеговой) меры на сффере $S_{\rho}^{N}$, т.е. $d \mu=d q d p$. Свойства бильярдной динамики зависят в значительной степени от кривизны гранищы $\partial Q$. В нашем случае $Q$ - полиэдр, следовательно, его гранища состоит из плоских граней нулевой кривизны. Прототипом такой системы является бильярд в многоугольнике. Хорошо известен (см. [5]) такой

$\Phi$ АКТ. Для бильярдов в многоугольниках и полиэдрах (многогранниках) (и, следовательно, для нашей механической модели поршня в идеальном газе) все показатели Ляпунова равны нулю и метрическая энтропия (Колмогорова-Синая) также равна нулю.

Системы с нулевьми показателями Ллпунова и с нулевой энтропией не рассматриваются как в полной мере хаотические, но они могут еще быть эргодическими. В самом деле, бильярды в многоугольниках общего положения әргодичны [14]. Кроме того, для многих неэргодических многоугольников фазовоепространство расслаивается на инвариантные поверхности, на которых динамика эргодична.

Насколько нам известно, для бильярдов в многогранниках высокой размерности не существует подобных результатов, но можно ожидать, что они тоже обладают подобными свойствами. То есть для многогранников общего положения они эргодичны или становятся таковыми после тривиальных редукций. В нашем случае бильярд 
в $Q$, возможно, эргодичен для типичных значений массы $M$; существует и другая возможность - фазовое пространство расслаивается на инвариантные подмногообразия, на которых динамика эргодична, и такие подмногообразия заполняют $\mathscr{M}$ достаточно плотно. В последнем случае едва ли можно отличить экспериментально такую неэргодическую систему от эргодической.

Следовательно, мы можем предположить, что наша система эргодична или очень близка к эргодической в указанном вьше смысле. Тогда поведение почти каждой траектории в действительности согласуется с инвариантной мерой $\mu$ независимо от начального состояния. В частности, для любых начальной плотности газа и распределения скоростей (заданных функцией $\pi_{0}(y, v)$, см. $\S 1$ ) гидродинамический режим для конечного $L$ будет хорошим приближением только на конечном интервале времени по сушеству, система будет релаксировать к тепловому равновесию. На самом деле, мы ожидаем, что в терминах "макроскопических" переменных, скажем, одночастичной функции распределения, система будет релаксировать к эффективному равновесию за определенное в (5.6) время $\tau_{\text {eq }}$, которое значительно меньше экспоненциально большого (по $L$ ) времени, требуемого эргодической теоремой. Так что, по существу, вопрос в том, как это время зависит от $L$. Согласно нашему обсуждению $\tau_{c} \sim \log L$, а $\tau_{\text {eq }} \sim L^{5 / 2}$.

В равновесии распределения координат $x_{i}$ и скоростей $v_{i}$ определяются мерой Лиувилля $\mu$, которая равномерна на фазовом пространстве. Физически интересными (и единственно наблюдаемыми) являются ее маргинальные меры, т.е. проекщии на подпространства более низкой размерности. Маргинальные меры скоростей для больших $N$ приблизительно нормальны.

В частности, каждая индивидуальная скорость $v_{i}$ сходится по распределению к распределению Максвелла (т.е. к нормальному распределению с нулевым средним и дисперсией $2 E / N=$ const). То же самое верно для “поршневой" компоненты скорости $\dot{x}_{0}=V \sqrt{M}$, поэтому скорость $V$ поршня будет нормально распределена со средним нуль и стандартным отклонением const $/ \sqrt{M}=\mathrm{const} / L$ при $L \rightarrow \infty$. В нашем случае $V$ имеет стандартное отклонение $\sqrt{7 / 24} / L \approx 0.5 / L$. Этот вьвод хорошо согласуется с теоремой 1.1 Холли и с нашими численными данными.

Равновесное распределение координат поршня $X$ также определяется проекцией равномерной меры $d q$ в $Q$ на этот раз на ось $x_{0}$. Прежде чем сделать это, избавимся от $M$ в определениях и $Q$, и $x_{0}$. Простая замена переменной $X=x_{0} / \sqrt{M}$ позволяет нам переопределить $Q$ как

$$
0 \leqslant x_{-N_{L}}, \ldots, x_{-1} \leqslant X \leqslant x_{1}, \ldots, x_{N_{R}} \leqslant L
$$

Более того, скейлинг $Y=X / L$ и $y_{i}=x_{i} / L$ дает более простое определение $Q$ :

$$
0 \leqslant y_{-N_{L}}, \ldots, y_{-1} \leqslant Y \leqslant y_{1}, \ldots, y_{N_{R}} \leqslant 1
$$

Мы получаем вариант так назьваемого броуновского моста. Интегрирование по переменным $y_{i}$ дает следуюшую равновесную плотность для $Y$ :

$$
f(Y)=c Y^{N_{L}}(1-Y)^{N_{R}}
$$


для $0<Y<1$, где $c$ - нормируюший множитель, который может быть вычислен явно:

$$
c^{-1}=\int_{0}^{1} Y^{N_{L}}(1-Y)^{N_{R}} d Y=\frac{N_{L} ! N_{R} !}{\left(N_{L}+N_{R}+1\right) !} .
$$

Асимптотически при $L \rightarrow \infty$ мы имеем $N_{L} \sim L^{3} / 2$ и $N_{R} \sim L^{3} / 2$. Предположим для простоты, что $N_{L}=N_{R}=N / 2$, и обозначим $N / 2$ через $K$, тогда

$$
c=\frac{(2 K+1) !}{(K !)^{2}} \simeq \frac{2 \cdot 4^{K} \sqrt{K}}{\sqrt{\pi}} .
$$

Положим $z=(Y-0.5) \sqrt{8 K}$. Плотность величины $z$ асимптотически дается формулой:

$$
\begin{aligned}
f(z) & =\frac{c}{\sqrt{8 K}}\left(0.5+\frac{z}{\sqrt{8 K}}\right)^{K}\left(0.5-\frac{z}{\sqrt{8 K}}\right)^{K} \\
& =\frac{c}{4^{K} \sqrt{8 K}}\left(1-\frac{z^{2}}{2 K}\right)^{K} \approx \frac{1}{\sqrt{2 \pi}} e^{-z^{2} / 2} .
\end{aligned}
$$

Таким образом, $Y$ оказьвается асимптотически гауссовской случайной величиной со средним 0.5 и дисперсией $(4 N)^{-1}=\left(4 L^{3}\right)^{-1}$. Следовательно, в равновесии

$$
|Y-0.5| \sim \frac{1}{2 L \sqrt{L}} \sim \frac{1}{2 \sqrt{N}} .
$$

Заметим, что эта оценка не зависит от массы поршня.

Далее, используя (5.7), можно легко вычислить вероятность того, что координата $Y$ поршня отклоняется от своего среднего значения 0.5 на фиксированную величину $d>0$, скажем, $d=0.1$ : эта вероятность меньше const $\cdot e^{-a N}$ для некоторого $a=a(d)>0$. Следовательно, мы наблюдали в эксперименте очень редкое событие, вероятность которого по $N$ экспоненциально мала. Если бы мы начали с равновесного состояния, такое наблюдение было бы практически невозможно. Но мы начали с состояния, описьваемого плотностью (5.1), которое само имеет вероятность меньшую, чем const $\cdot e^{-b N}, b>0$, относительно равновесной меры $\mu$. Итак, мы всего лишш наблюдали, как одно очень маловероятное начальное состояние переходит в другое столь же маловероятное состояние вдоль его очень медленного движения к равновесному состоянию. Было бы интересно понять, почему система "выбирает" такой странньй путь перехода к равновесию, т.е. почему, начиная с состояния, задаваемого “двугорбым” распределением (5.1), эта система ведет себя как затухающий гармонический осциллятор на большом временном интервале с начальньми осциллящиями, достигающими 1/10 размера системы.

Мы продолжаем работать над этой проблемой и сообщим результаты в отдельной статье [2]. Ниже мы опишем в обших чертах нашу программу и упомянем о некоторых предварительных наблюдениях. Для простоты мы предположим, что $\pi_{0}(y, v)=$ $\pi_{0}(|v|)$ и $X(0)=L / 2, V(0)=0$. Следствие 2.13 гарантирует, что гидродинамические уравнения имеют тривиальное решение $Y(\tau) \equiv 0.5, W(\tau) \equiv 0$ и $\pi(y, v, t) \equiv \pi_{0}(y, v)$ для всех $\tau>0$. Так как начальная конфигурация частиц является случайной реализацией пуассоновского процесса с плотностью $\pi_{0}(y, v)$, "реальная" (эмпирическая) плотность частищ, такая, как та, что показана на рис. 10 при $\tau=0$, не совпа- 
дает в точности с $\pi_{0}(y, v)$. Случайные флуктуации эмпирической плотности имеют порядок $O(1 / L)$, поэтому "реальное" начальное распределение частиц можно представлять себе как малое возмущение функции $\pi_{0}(y, v)=\pi_{0}(|v|)$ и записьвать как $\pi_{0}(|v|)+\varepsilon \pi_{1}(y, v) \mathrm{c} \varepsilon=1 / L$ и некоторой (случайной) функцией $\pi_{1}(y, v)$ порядка единицы.

Мы высказьваем гипотезу, что эволюция механической системы тесно следует решениям гидродинамических уравнений (H1)-(H4) с возмушенной начальной плотностью $\pi_{0}(|v|)+\varepsilon \pi_{1}(y, v)$ скорее чем стационарному решению, соответствующему невозмушенной плотности $\pi_{0}(|v|)$. Этим объясняется значительное различие в поведении механической системы и стационарного решения, если последнее неустойчиво. В частности, две траектории, которые в начале $\varepsilon$-близки (в нашем случае, $\varepsilon=1 / L$ ), могут отклониться друг от друга экспоненщиально быстро со временем $\tau$ и ко времени $\tau \sim-\log \varepsilon=\log L$ они будут вьглядеть совершенно по-разному. Это согласовывалось бы с нашими экспериментальными наблюдениями и с оценкой (5.5) времени $\tau_{c} \sim \log L$ начала "неустойчивости".

Чтобы проверить нашу гипотезу, мы решили гидродинамические уравнения (H1)-(Н4) численно с возмущенной начальной плотностью, полученной добавлением к (5.1) функции, $\varepsilon$-малой в $L^{1}$-метрике (с $\left.\varepsilon \approx 0.01\right)$. Мы обнаружили, что соответствуюшее решение поразительно напоминает эволюцию механической системы, описанной вьше. В частности, координата и скорость поршня испытьвают большие почти гармонические колебания в интервале $10<\tau<30$. Соответствуюшие графики положения и скорости поршня вдоль возмущенных решений уравнений $(\mathrm{H} 1)-(\mathrm{H} 4)$ оказались почти неотличимыми от графиков на рис. 6 и 7. Следовательно, поведение механической системы может быть отслежено по поведению возмушенных решений гидродинамических уравнений, и неустойчивость последнего становится важным фактором.

Когда мы заканчивали эту статью, мы получили письмо от Е. Каллиоти и Е. Презутти, которые (а) доказали устойчивость решений гидродинамических уравнений $(\mathrm{H} 1)-(\mathrm{H} 4)$, если $\pi_{0}(|v|)$ - монотонно невозрастающая функция от $|v|$, т.е. $\pi_{0}^{\prime}(|v|) \leqslant 0$, и (б) предположили, что эти решения могут быть неустойчивы для нашего класса немонотонных $\pi_{0}(|v|)$. Мы проверили предположение (б) для нашей специальной плотности (5.1) и обнаружили, что это предположение правильно; мы доказали, что малые возмущения растут экспоненциально по $\tau$.

Обратно, просчитав динамику частиц с невозрастающей начальной плотностью $\pi_{0}(|v|)$, мы увидели, что осцилляции действительно исчезли (мы исследовали случай равномерно "гладкой" начальной функции $\pi_{0}(|v|)=1$ для $|v| \leqslant v_{\max }$ и случай треугольной начальной функции $\left.\pi_{0}(|v|)=1-|v| / v_{\max }\right)$. С другой стороны, распределение скорости частищ продолжало приближаться к максвелловскому, хотя и более медленно.

Наконец, мы теперь опишем другие остаюшиеся открытыми проблемы, связанные с динамикой поршня.

1. Ясно, что перестолкновения частиц газа с поршнем оказьвают "разрушительные" воздействия на динамику системы. Однако нам нужно различать два типа перестолкновений.

Мы скажем, что перестолкновение частищы газа с поршнем является длинным, если частица ударяется об одну из стенок $x=0$ или $x=L$ между двумя последователь- 
ными столкновениями с поршнем. В противном случае перестолкновение назьвается коротким. Длинные перестолкновения требуют некоторого времени, так как частица должна пройти весь путь до стенки, оттолкнуться от нее и затем вернуться назад к поршню перед тем, как столкнуться с ним снова. Короткие перестолкновения могут быстро следовать друг за другом.

Мы наложили на скорость условие обрезания (Р4) для того, чтобы избежать любого перестолкновения, по крайней мере, за некоторый начальный период времени (которьй мы назвали интервалом без перестолкновений). Более точно, верхняя граница $v_{\max }$ гарантирует отсутствие длинных перестолкновений. Без этого нам пришлось бы иметь дело с как угодно быстрьми частицами, которые пробегали бы между поршнем и стенками много раз в течение любого интервала $(0, \tau)$. С другой стороны, нижняя гранища $v_{\min }$ была введена для того, чтобы исключить короткие перестолкновения.

Существуют, однако, разумные основания считать, что короткие перестолкновения могут не быть столь разрушительными для динамики поршня. В самом деле, пусть частица испытьвает два или более последовательных столкновений с поршнем через короткие отрезки времени (т.е. без соударений со стенкой в этих промежутках). Это может произойти в двух случаях: (i) скорость частищы будет очень близка к скорости поршня; или (iі) скорость поршня очень быстро изменится. Последнее вряд ли может случиться, так как детерминистское ускорение поршня очень мало (ср. с теоремой 2.12(c)). В случае (i) перестолкновения должны очень слабо воздействовать на скорость поршня согласно правилу (1.2), так что их можно вполне проигнорировать, как это уже было сделано в некоторых более ранних исследованиях [13], [8].

Следовательно, мы можем надеяться, что наши результаты обобщаются на распределения скоростей, не отделенных от нуля, т.е. на $v_{\min }=0$.

2. В нашей работе $L$ играет двойную роль: оно параметризует массу поршня $\left(M \sim L^{2}\right)$ и является длиной сосуда $(0 \leqslant x \leqslant L)$. Эта двойственность проистекает из нашего предложения о том, что сосуд имеет кубическую форму.

Однако наша модель, по сушеству, одномерна, и масса поршня $M$ и длина $0 \leqslant x \leqslant L$ интервала могут рассматриваться как два независимых параметра. В частности, мы можем предположить, что сосуд имеет бесконечную длину в направлении $x$ (т.е. $m a-$ кое $L$ бесконечно), но масса поршня все же конечна и задается как $M \sim L^{2}$ (это $L$ есть размер сосуда в направлениях $y$ и $z$ ). В этом случае перестолкновения с поршнем не происходят, пока его скорость остается малой. Поэтому наш интервал без перестолкновений по существу бесконечен. Как следствие, теорему 1.2 можно обобшить на произвольно большие времена. А именно, для любого $T>0$ мы можем доказать сходимость по вероятности:

$$
\mathrm{P}\left(\sup _{0 \leqslant \tau \leqslant T}\left|Y_{L}(\tau, \omega)-Y(\tau)\right| \leqslant C_{T} \frac{\ln L}{L}\right) \rightarrow 1
$$

и

$$
\mathrm{P}\left(\sup _{0 \leqslant \tau \leqslant T}\left|W_{L}(\tau, \omega)-W(\tau)\right| \leqslant C_{T} \frac{\ln L}{L}\right) \rightarrow 1
$$

при $L \rightarrow \infty$, где $C_{T}>0$ - постоянная, а $Y(\tau)$ и $W(\tau)=\dot{Y}(\tau)$ - решения гидродинамических уравнений из $\S 2$. 
3. Таким же образом, как и вьше, мы можем предположить, что сосуд $d$-мерен с $d \geqslant 4$. Тогда масса поршня и плотность частиц будут пропорциональны $L^{d-1}$ вместо $L^{2}$.

Когда $d$ велико, плотность газа очень высокая в $(x, v)$-плоскости. Это приводит к значительно лучшему контролю за флуктуациями распределения частиц и траекторией поршня. Например, в интервале без перестолкновений траектория поршня $L^{-(d-1) / 2}$-близка к его детерминистской траектории. Это простая модифиикация результатов нашего $\S 3$. В течение интервала одного перестолкновения траектория поршня $L^{-(2 d-5) / 7}$-близка к ее детерминистской траектории. Это может быть показано методами, развитыми в $\S 4$, но требует некоторой дополнительной работы. Кроме того, методы и результаты этого параграфа могут быть распространены на интервал $\left(\tau_{k}, \tau_{k+1}\right)$ с $k$ перестолкновениями для любого $k \geqslant 1$. Можно показать, что существует такое $d_{k} \geqslant 3$, что для всех $d \geqslant d_{k}$

$$
\mathrm{P}\left(\sup _{\tau_{k}<\tau<\tau_{k+1}}\left|W_{L}(\tau, \omega)-W(\tau)\right| \leqslant L^{-b}\right) \rightarrow 1
$$

при $L \rightarrow \infty$, где $b>0$ зависит от $k$ и $d$. Это обобшение, однако, требует совсем других методов, которые выходят за рамки этой статьи. Вьвод заключается в том, что поршень высокой размерности более устойчив, чем поршень низкой размерности.

Было бы интересно исследовать другие модификации нашей модели, приводяшие к более устойчивым режимам. Например, пусть начальная плотность $\pi_{0}(y, v)$ газа зависит от множителя $a=\varepsilon L^{2}$ таким образом, что $\pi_{0}(y, v)=a^{-1} \rho(y, v)$, где $\rho(y, v)-$ фиксированная функция. Тогда плотность частиц возрастает при $a \rightarrow 0$. Это другой путь для увеличения плотности частищ без изменения размерности. Можно надеяться, что и в этом случае лучший контроль за случайными флуктуашиями осушествим.

Благодарности. Мы благодарим Й. Пьясецки, Ч. Грубера, Е. Либа, М. Мансура, В. Яхота, Н. Шимани за многочисленные полезные обсуждения и ценные предложения. Н. Чернов был частично поддержан NSF (грант DMS-0098788), Д. Лебовищ частично поддержан NSF (грант DMR-9813268) и фондом Воздушных Сил (грант F49620-01-0154). Я. Синай частично поддержан NSF (грант DMS-9706794). Эта работа была завершена, когда Л. Лебовиц и Н. Чернов находились в Институте перспективных исследований и были частично поддержаны NSF (грант DMS-9729992).

\section{Приложение}

В этом разделе мы выводим вероятностные оценки распределения частиц газа и их скоростей. Число частиц $K=N_{D}$ в любой области $D$ на $(x, v)$-плоскости в момент времени $t$ есть пуассоновская случайная величина. Нам нужны оценки для его больших уклонений. Может быть, некоторые из наших оценок известны в теории вероятностей; но ради полноты мы включаем их доказательство.

ЛЕмма А.1. Пусть $K$ - пуассоновская случайная величина с параметром $\lambda>0$. Тогда для любого $A>\lambda$

$$
\mathrm{P}(K>A) \leqslant e^{A-\lambda-A \ln (A / \lambda)}
$$

и для любого $A<\lambda$

$$
\mathrm{P}(K<A) \leqslant e^{A-\lambda-A \ln (A / \lambda)} .
$$


ДоКАЗАТЕЛЬСТвО. Производящая функщия моментов величины $K$ есть

$$
\varphi_{K}(t)=\mathrm{E}\left(e^{t K}\right)=e^{\lambda\left(e^{t}-1\right)} .
$$

Сначала пусть $A>\lambda$. Тогда очевидно, что для всех $t>0$

$$
\varphi_{K}(t) \geqslant e^{A t} \mathrm{P}(K>A) .
$$

Отсюда для всех $t>0$

$$
\mathrm{P}(K>A) \leqslant e^{\lambda\left(e^{t}-1\right)-A t} .
$$

Выражение в правой части достигает минимума в точке

$$
t=\ln (A / \lambda)>0 .
$$

Это доказьвает первую часть леммы.

Пусть теперь $A<\lambda$. Тогда для всех $t<0$

$$
\varphi_{K}(t) \geqslant e^{A t} \mathrm{P}(K<A) .
$$

Отсюда для всех $t<0$

$$
\mathrm{P}(K<A) \leqslant e^{\lambda\left(e^{t}-1\right)-A t} .
$$

Выражение в правой части достигает минимума в точке

$$
t=\ln (A / \lambda)<0
$$

Это доказьвает вторую часть леммы.

ЛЕмма А.2. Пусть $K$ - пуассоновская случайная величина с параметром $\lambda>0$. Для любого $b>0$ существует такое $c>0$, что для всех $0<B<b \sqrt{\lambda}$

$$
\mathrm{P}(|K-\lambda|>B \sqrt{\lambda}) \leqslant 2 e^{-c B^{2}} .
$$

ДоКАЗАТЕЛЬСТво. Прямое применение предыдушей леммы дает

$$
\mathrm{P}(|K-\lambda|>B \sqrt{\lambda}) \leqslant 2 e^{-B^{2} g(q)},
$$

где

$$
g(q)=\frac{(1+q) \ln (1+1)-q}{q^{2}}=\frac{\int_{0}^{q} \ln (1+s) d s}{2 \int_{0}^{q} s d s}
$$

и $q=B / \sqrt{\lambda}_{\text {в }}$ (А.1). Прямой проверкой можно убедиться, что функция $g(q)$ положительна и строго монотонно убывает в интервале $0<q<\infty$. Мы завершаем доказательство, полагая $c=g(b)$. 
Лемма А.3. Пусть $\lambda_{0}>0$ и а $>0$. Тогда для всех достаточно больиих $L>0$ и каждой пуассоновской случайной величины $K$ с параметром $\lambda \geqslant \lambda_{0}$

$$
\mathrm{P}(|K-\lambda|>a \sqrt{\lambda} \ln L) \leqslant L^{-d \ln \ln L},
$$

əде $d=a \sqrt{\lambda_{0}} / 2$.

ДокАЗАТЕЛЬСТво. Подстановка в (А.1) вместо $B$ значения $a \ln L$ дает

$$
\mathrm{P}(|K-\lambda|>a \sqrt{\lambda} \ln L) \leqslant 2 e^{-(a \ln L)^{2} g\left(a \ln L / \sqrt{\lambda_{0}}\right)},
$$

где $g(q)$ определено в (А.2). Заметим, что для больших $L$

$$
g\left(\frac{a \ln L}{\sqrt{\lambda_{0}}}\right) \sim \frac{\sqrt{\lambda_{0}} \ln \ln L}{a \ln L} .
$$

Это завершает доказательство.

ЗАмечАниЕ. В большей части наших применений $\lambda$ является большим, порядка $\lambda \sim L^{b}$ с некоторьм $b>0$. Следовательно, $\ln L$ мал по сравнению с $\sqrt{\lambda}$.

СЛЕДСтвИЕ А.4. Пусть $\lambda_{0}>0$ и $а>0$. И пусть $K-$ пуассоновская случайная величина с параметром $\lambda \leqslant \lambda_{0}$. Тогда для всех достаточно больших $L>0$

$$
\mathrm{P}(K>a \ln L) \leqslant L^{-d \ln \ln L},
$$

əде $d=a / 2$.

ДокАЗАТЕЛЬСТвО. Случай $\lambda=\lambda_{0}$ легко следует из предыдущей леммы. Если же $\lambda<\lambda_{0}$, то событие $K>a \ln L$ даже менее вероятно, чем при $\lambda=\lambda_{0}$.

Далее, нам нужно изучить другую случайную величину, связанную с процессом Пуассона. Для любой области $D$ на $(x, v)$-плоскости рассмотрим сумму скоростей

$$
Z=Z_{D}=\sum_{(x, v) \in D} v
$$

частиц из $D$ в момент времени 0 . Мы предположим, что $D \subset\left\{v_{\min }<v<v_{\max }\right\}$ (случай $D \subset\left\{-v_{\max }<v<-v_{\min }\right\}$ совершенно симметричен и аналогичен первому). Проектируя область $D$ на ось $v$, мы получим пуассоновский процесс на интервале

$$
I=\left(v_{\min }, v_{\max }\right)
$$

с плотностью

$$
\pi(v)=L^{2} \int_{D \cap\{u=v\}} p_{L}(x, u) d x .
$$

Теперь случайная величина $Z$ может быть описана следуюшим образом. 
Рассмотрим одномерный пуассоновский процесс с плотностью $\pi(v)$ на интервале $I$. Это означает, что для любого подьнтервала $J \subset I$ число точек в $J$, назовем его $N_{J}$, есть пуассоновская случайная величина со средним

$$
\mathrm{E}\left(N_{J}\right)=\int_{J} \pi(v) d v
$$

Каждая реализация $\omega$ этого процесса является конечным подмножеством в $I$. Рассмотрим случайную величину

$$
Z(\omega)=\sum_{v \in \omega} v
$$

Мы будем называть $Z$ интегрированной пуассоновской случайной величиной.

Если мы зафиксируем большое $n \geqslant 1$ и разобьем $I$ на малые интервалы

$$
\Delta_{i}=I \cap\left[\frac{i}{n}, \frac{i+1}{n}\right)
$$

$i=0,1,2, \ldots$, мы можем, очевидно, оценить $Z$ следующим образом:

$$
\sum_{i} \frac{i}{n} N_{\Delta_{i}} \leqslant Z<\sum_{i} \frac{i+1}{n} N_{\Delta_{i}}
$$

где $N_{\Delta_{i}}$ - число случайных точек в интервале $\Delta_{i}$. Заметим, что $N_{\Delta_{i}}$ - независимые пуассоновские случайные величины с параметрами

$$
\lambda_{i}=\mathrm{E}\left(N_{\Delta_{i}}\right)=\int_{\Delta_{i}} \pi(v) d v \text {. }
$$

Поэтому производящая функщия $\varphi_{Z}(t)=\mathrm{E}\left(e^{t Z}\right)$ моментов величины $Z$ ограничивается следующим образом:

$$
\exp \left[\sum_{i} \lambda_{i}\left(e^{t \frac{i}{n}}-1\right)\right] \leqslant \mathrm{E}\left(e^{t Z}\right)<\exp \left[\sum_{i} \lambda_{i}\left(e^{t \frac{i+1}{n}}-1\right)\right]
$$

Устремляя $n \mathrm{k} \infty$, мы получим, что

$$
\varphi_{Z}(t)=\mathrm{E}\left(e^{t Z}\right)=\exp \left[\int_{I}\left(e^{t v}-1\right) \pi(v) d v\right]
$$

Воспользовавшись (А.3), легко найти среднее значение

$$
\mathrm{E}(Z)=\mu_{Z}=\int_{I} v \pi(v) d v
$$

и дисперсию

$$
\mathrm{D}(Z)=\sigma_{Z}^{2}=\int_{I} v^{2} \pi(v) d v
$$


Отметим, что $Z$ связана с пуассоновской случайной величиной $K=N_{I}$ с параметpom

$$
\lambda_{Z}=\int_{I} \pi(v) d v
$$

В частности,

$$
v_{\min } K \leqslant Z \leqslant v_{\max } K
$$

следовательно,

$$
v_{\min } \lambda_{Z} \leqslant \mu_{Z} \leqslant v_{\max } \lambda_{Z}
$$

Мы имеем также

$$
v_{\min }^{2} \lambda_{Z} \leqslant \sigma_{Z}^{2} \leqslant v_{\max }^{2} \lambda_{Z}
$$

Случайная величина допускает оценки больших уклонений, подобные тем, которые мы нашли для пуассоновских случайных величин:

Лемма А.5. Для любого $b>0$ существует $c>0$ (определяемое значениями $\left.b, v_{\min }, v_{\max }\right)$ такое, что для любой интегрированной пуассоновской случайной величины $Z$ и всех $0<B<b \sigma_{Z}$

$$
\mathrm{P}\left(\left|Z-\mu_{Z}\right|>B \sigma_{Z}\right) \leqslant 2 e^{-c B^{2}} .
$$

ДоКАЗАТЕЛЬСТВо. Положим для краткости $\mu=\mu_{Z}$ и $\sigma=\sigma_{Z}$. Мы покажем, что

$$
\mathrm{P}(Z>\mu+B \sigma) \leqslant e^{-c B^{2}}
$$

(та же оценка для $\mathrm{P}(Z<\mu-B \sigma)$ доказывается подобным образом, как мы это сделали в лемме А.1). Для всех $t>0$

$$
\varphi_{Z}(t) \geqslant e^{(\mu+B \sigma) t} \mathrm{P}(Z>\mu+B \sigma)
$$

поэтому

$$
\mathrm{P}(Z>\mu+B \sigma) \leqslant \exp \left[\int_{I}\left(e^{t v}-1\right) \pi(v) d v-(\mu+B \sigma) t\right] .
$$

Мы делаем подстановку $t=B s / \sigma \mathrm{c} s>0$, которое будет выбрано позже, и разлагаем $e^{t v}$ в ряд Тейлора:

$$
\mathrm{P}(Z>\mu+B \sigma) \leqslant \exp \left[\int_{I}\left(\sum_{n=1}^{\infty} \frac{(B s v)^{n}}{\sigma^{n} n !}\right) \pi(v) d v-\frac{B s \mu}{\sigma}-B^{2} s\right] .
$$

Первые два члена с $n=1$ и $n=2$ дают

$$
\int_{I} \frac{B s v}{\sigma} \pi(v) d v=\frac{B s \mu}{\sigma}
$$

в силу (А.5) и

$$
\int_{I} \frac{(B s v)^{2}}{2 \sigma^{2}} \pi(v) d v=\frac{B^{2} s^{2}}{2}
$$

в силу (А.6). 
Таким образом,

$$
\mathrm{P}(Z>\mu+B \sigma) \leqslant \exp \left[-B^{2}\left(s-\frac{s^{2}}{2}-s^{2} \sum_{n=3}^{\infty} \frac{(B s / \sigma)^{n-2} \int_{I} v^{n} \pi(v) d v}{n ! \sigma^{2}}\right)\right]
$$

Считая, что $B / \sigma<b$, и используя (А.6), мы получим, что

$$
\sum_{n=3}^{\infty} \frac{(B s / \sigma)^{n-2} \int_{I} v^{n} \pi(v) d v}{n ! \sigma^{2}}<\sum_{k=1}^{\infty} \frac{(s b)^{k} v_{\max }^{k+2}}{k ! v_{\min }^{2}}<\frac{v_{\max }^{2}}{v_{\min }^{2}}\left(e^{s b v_{\max }}-1\right)
$$

Теперь при достаточно малом $s$ мы выберем

$$
c:=s-\frac{s^{2}}{2}-s^{2} \frac{v_{\max }^{2}}{v_{\min }^{2}}\left(e^{s b v_{\max }}-1\right)>0 .
$$

Это доказьвает (А.10) и, следовательно, лемму.

Лемма А.6. Пусть $\lambda_{0}>0$. Для всех достаточно больших $L>0$ и любой интегрированной пуассоновской случайной величины $Z$ с $\lambda_{Z} \geqslant \lambda_{0}$

$$
\mathrm{P}\left(\left|Z-\mu_{Z}\right|>\sigma_{Z} \ln L\right) \leqslant L^{-d \ln \ln L},
$$

где $d>0$ - постоянная, определяемая по $\lambda_{0}, v_{\min }, v_{\max }$.

ДокАЗАТЕЛЬСТВо. Пусть

$$
b=\frac{2 v_{\max }}{v_{\min }^{2}} .
$$

Если $\ln L<b \sigma_{Z}$, результат легко вытекает из предыдущей леммы.

Предположим теперь, что

$$
\ln L \geqslant b \sigma_{Z}
$$

Из неравенств (А.8) и (А.9) получаем, что

$$
\lambda_{Z} \leqslant \frac{\sigma_{Z}^{2}}{v_{\min }^{2}} \leqslant \frac{(\ln L)^{2}}{b^{2} v_{\min }^{2}}
$$

и, следовательно,

$$
\mu_{Z} \leqslant v_{\max } \lambda_{Z} \leqslant \frac{v_{\max }(\ln L)^{2}}{b^{2} v_{\min }^{2}}
$$

и также

$$
\mu_{Z} \leqslant v_{\max } \lambda_{Z} \leqslant \frac{v_{\max }}{v_{\min }^{2}} \sigma_{Z}^{2}
$$

Умножение (А.12) на (А.13) и извлечение квадратного корня дает, что

$$
\mu_{Z} \leqslant \frac{v_{\max }}{b v_{\min }^{2}} \sigma_{Z} \ln L=\frac{1}{2} \sigma_{Z} \ln L .
$$


Из того, что $Z$ - положительная случайная величина, мы получаем, что

$$
\mathrm{P}\left(\left|Z-\mu_{Z}\right|>\sigma_{Z} \ln L\right)=\mathrm{P}\left(Z>\mu_{Z}+\sigma_{Z} \ln L\right) \leqslant \mathrm{P}\left(Z>\sigma_{Z} \ln L\right) .
$$

Более того, комбинирование (А.14) с (А.11) и (А.9) приводит к соотношениям

$$
\frac{1}{2} \sigma_{Z} \ln L=\frac{v_{\max }}{b v_{\min }^{2}} \sigma_{Z} \ln L \geqslant \frac{v_{\max }}{v_{\min }^{2}} \sigma_{Z}^{2} \geqslant v_{\max } \lambda_{Z},
$$

а также в силу (А.9) к неравенству

$$
\frac{1}{2} \sigma_{Z} \ln L \geqslant \frac{1}{2} v_{\min } \sqrt{\lambda_{Z}} \ln L
$$

Тогда, поскольку $Z \leqslant v_{\max } K$ в силу (А.7), мы имеем

$$
\begin{aligned}
\mathrm{P}\left(Z>\sigma_{Z} \ln L\right) & \leqslant \mathrm{P}\left(Z>v_{\max } \lambda_{Z}+\frac{1}{2} v_{\min } \sqrt{\lambda_{Z}} \ln L\right) \\
& \leqslant \mathrm{P}\left(K>\lambda_{Z}+\frac{v_{\min }}{2 v_{\max }} \sqrt{\lambda_{Z}} \ln L\right) .
\end{aligned}
$$

Теперь результат следует из леммы А.3.

СЛЕДСТВИЕ А.7. Пусть $\lambda_{0}>0$, и пусть $Z$ - интегрированная пуассоновская величина с $\lambda_{Z} \leqslant \lambda_{0}$. Тогда для всех достаточно больиих $L>0$

$$
\mathrm{P}(Z>\ln L) \leqslant L^{-d \ln \ln L},
$$

где $d>0$ определяется по $\lambda_{0}, v_{\max }, v_{\min }$.

Следствие А.7 непосредственно вытекает из следствия А.4.

\section{СПИСОК ЛИТЕРАТУРЫ}

[1] L. Boltzmann. Reply to Zermelo's Remarks on the Theory of Heat // Ann. Phys. 1896. V. 57. P. 773; reprinted and translated as Chapter 8 in S. G. Brush. Kinetic Theory. Oxford: Pergamon, 1996.

[2] E. Cagliotti, N. Chernov, J.L. Lebowitz. Instability of piston dynamics // www.math.uab.edu/chernov/papers/pubs.html.

[3] H. B. Callen. Thermodynamics. New York: Wiley, 1963.

[4] N. Chernov, J. L. Lebowitz. Dynamics of a Massive Piston in an Ideal Gas: Oscillatory Motion and Approach to Equilibrium // J. Statist. Phys. (to appear); // www.math.uab.edu/ chernov/papers/pubs/.

[5] N. Chernov. Entropy, Lyapunov exponents, and mean-free path for billiards // J. Statist. Phys. 1997. V. 88. №1-2. P. 1-29.

[6] И.П. Корнфельд, Я.Г. Синай, С.В. Фомин. Эргодическая теория. М.: Наука, 1980 .

[7] B. Crosignani, P. Di Porto, M. Segev. Approach to thermal equilibrium in a system with adiabatic constraints // Amer. J. Phys. 1996. V. 64. P. 610-613.

[8] D. Dürr, S. Goldstein, J. L. Lebowitz. A mechanical model of Brownian motion // Comm. Math. Phys. 1981. V. 78. № 4. P. 507-530.

[9] Р. Фейнман, Р. Лейтон, М. Сэндс. Фейнмановские лекции по физике. М.: Наука, 1976-1979. 
[10] Ch. Gruber. Thermodynamics of systems with internal adiabatic constraints: time evolution of the adiabatic piston // European J. Phys. 1999. V. 20. № 4. P. 259-266.

[11] Ch. Gruber, L. Frachebourg. On the adiabatic properties of a stochastic adiabatic wall: Evolution, stationary non-equilibrium, and equilibrium states // Phys. A. 1999. V. 272. P. $392-428$.

[12] Ch. Gruber, J. Piasecki. Stationary motion of the adiabatic piston // Phys. A. 1999. V. 268. P. 412-423.

[13] R. Holley. The motion of a heavy particle in an infinite one dimensional gas of hard spheres // Z. Wahrscheinlichkeitstheor. verw. Geb. 1971. V. 17. P. 181-219.

[14] S. Kerckhoff, H. Masur, J. Smillie. Ergodicity of billiard flows and quadratic differentials // Ann. of Math. (2). 1986. V. 124. P. 293-311.

[15] E. Kestemont, C. van den Broeck, M. Mansour. The "adiabatic" piston: and yet it moves // Europhys. Lett. 2000. V. 49. P. 143-149.

[16] А. Н. Колмогоров, В. М. Тихомиров. $\varepsilon$-энтропия и $\varepsilon$-емкость множеств в функционалшных пространствах // УМН. 1959. Т. 14. № 2. С. 3-86.

[17] R. Kubo. Statistical Mechanics. Amsterdam: North-Holland, 1965.

[18] Л.Д. Ландау, Е. М. Лиффшиц. Статистическая физика. М.: Наука, 1976.

[19] J. L. Lebowitz, J. Piasecki, Ya. Sinai. Scaling dynamics of a massive piston in an ideal gas // Hard Ball Systems and Lorentz Gas. Berlin: Springer-Verlag, 2000. P. 217-227. (Encyclopaedia Math. Sci. V. 101.)

[20] J. L. Lebowitz. Stationary non-equilibrium Gibbsian ensembles // Phys. Rev. (2). 1959. V. 114. P. 1192-1202.

[21] E. Lieb. Some problems in statistical mechanics that I would like to see solved // Phys. A. 1999. V. 263. № 1-4. P. 491-499.

[22] R. Lupton. Statistics in Theory and Practice. Princeton, NJ: Princeton Univ. Press, 1993.

[23] M. Matsumoto, T. Nishimura. Mersenne Twister: A 623-dimensionally equidistributed uniform pseudorandom number generator // ACM Trans. Model. Comput. Simul. 1998. V. 8. № 1. P. 3-30.

[24] E. Nelson. Dynamical Theories of Brownian Motion. Princeton, NJ: Princeton Univ. Press, 1967.

[25] J. Piasecki, Ch. Gruber. From the adiabatic piston to macroscopic motion induced by fluctuation // Phys. A. 1999. V. 265. P. 463-472.

[26] А. Н. Тихонов, А. Б. Васильева, А. Г. Свешников. Дифференциальные уравнения. M.: Наука, 1985.

Ратгерский университет, США,

Институт перспективных исследований, Принстон, США;

Поступила в редакцию

Принстонский университет, США;

Университет Алабамы в Бирмингеме, США,

Институт перспективных исследований, Принстон, США

16.05 .2002 